\title{
Microbial community dynamics \\ in \\ traditionally fermented milk
}

Anneloes E Groenenboom 


\section{Thesis committee}

\section{Promotors}

Prof. Dr E.J. Smid

Personal Chair at the Food Microbiology Group

Wageningen University \& Research

Prof. Dr B.J. Zwaan

Professor of Genetics

Wageningen University \& Research

\section{Co-promotors}

Dr S.E. Schoustra

Assistant professor at the Laboratory of Genetics

Wageningen University \& Research

Visiting professor at the Department of Food Science and Nutrition

University of Zambia

Dr A.R. Linnemann

Assistant professor at Food Quality and Design

Wageningen University \& Research

\section{Other members}

Dr M.G.J. de Vos, University of Groningen

Dr D.E. Rozen, Leiden University

Prof. Dr J. Hugenholtz, University of Amsterdam

Prof. Dr H. Smidt, Wageningen University \& Research

This research was conducted under the auspices of the Graduate School for Product Ecology and Resource Conservation. 


\title{
Microbial community dynamics
}

in

\author{
traditionally fermented milk
}

\section{Anneloes E Groenenboom}

\section{Thesis}

submitted in fulfilment of the requirements for the degree of doctor at Wageningen University

by the authority of Rector Magnificus,

Prof. Dr A.P.J. Mol,

in the presence of the

Thesis Committee appointed by the Academic Board

to be defended in public

on Friday 21 June 2019

at $1: 30$ PM in the Aula. 
Anneloes E Groenenboom

Microbial community dynamics in traditionally fermented milk, 146 pages

PhD thesis, Wageningen University, Wageningen, The Netherlands (2019) With references, with summaries in English and Dutch

ISBN 978-94-6343-966-4

DOI https://doi.org/10.18174/476594 


\section{Table of contents}

Chapter 1

Introduction

Chapter 2

18

Microbial communities from spontaneously fermented foods as model

system to study eco-evolutionary dynamics.

Chapter 3

34

Robust sampling and preservation of DNA for microbial community profiling in field experiments.

\section{Chapter 4}

Microbial population dynamics during traditional production of Mabisi, a spontaneously fermented milk product from Zambia. A field trial.

\section{Chapter 5}

Does change in bacterial species composition of natural communities reflect adaptation to a new environment?

\section{Chapter 6}

Bacterial community dynamics in Lait caillé, a traditional product of spontaneous fermentation from Senegal.

\section{Chapter 7}

General discussion

References

Summary

Acknowledgements

List of publications

About the author 
Chapter 1. 


\section{Introduction}

Anneloes E Groenenboom 


\section{Introduction}

This thesis is about Mabisi. In the western world, and maybe anywhere but Zambia, Mabisi is an unknown product. In this country in the middle of Africa, however, Mabisi is a phenomenon; a widely appreciated fermented milk product, which is consumed almost daily by men, women, and children from a very young age. What makes this fermented milk so interesting that I studied it for four years? It is the diversity we find in the bacterial communities that make Mabisi. This PhD thesis is part of a larger project funded by NWO WOTRO on enhancing nutrition security though traditional fermented products (1). In this thesis I would like to show the microbial communities that are responsible for the fermentation and how we can use the constituting bacteria to learn about bacterial community dynamics over time. This was done by combining three disciplines: 1) Food Microbiology, on the conversion of compounds by bacteria during spontaneous fermentation; 2) Evolution, on the changes in the fermenting community over time under selection pressure; 3) Ecology, on the roles and niches bacteria take within the fermenting community (Figure 1.1). My work does not only advance science; results of this project will also be used for improvements in production and legal regulation around Mabisi as well as upscaling production processes to ensure widespread availability of Mabisi thereby improving public health. This final aspect I will discuss in the General Discussion. For now, I would like to start by introducing Mabisi.

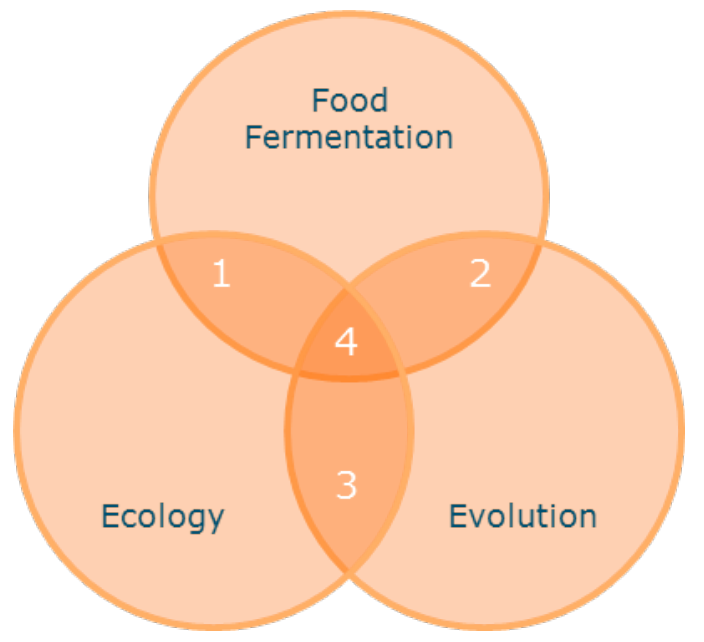

Figure 1.1 My thesis, linking three disciplines in one PhD project. Combining disciplines has added value compared to isolated disciplinary studies: 1 ) Using microbial communities from fermented foods is a great way to tackle questions in less trackable microbiota (2), 2) As sequencing costs are decreasing, analysing genetic changes in microbes used for fermentation becomes more accessible $(3), 3)$ The eco-evolutionary dynamics are studied more and more (4), and 4) in this thesis I combine these disciplines for a complete understanding of community dynamics $(5,6)$.

\section{Mabisi}

In many African countries local fermented products with unique properties are produced. Those products offer a great potential to enhance the nutritional status and livelihood of local producers and consumers (7). Zambia also has numerous endogenous fermented products (5), comparable to well known (Western) equivalents such as yoghurt, wine, beer, and sauerkraut. 
Mabisi is a non-alcoholic, milk-based product, which is consumed mainly in rural areas and is sold at local markets (Figure 1.2). Producers are almost exclusively women, who produce Mabisi according to ancient traditions. Overall, Mabisi has a slightly firm consistency, a mildly sour taste and a perceived shelf-life of a few weeks at ambient temperatures. It is made from raw cow milk that is kept in a calabash of up to 40 litres to allow fermentation over the course of two days at ambient temperatures (Figure 1.3), and variations of this production method are prevalent. For instance, as calabashes are becoming less available, cheaper fermentation vessels are found in plastic buckets and metal milk containers (Figure 1.2). Also, there are variations in the fermentation process itself, such as churning before and during fermentation, draining part of the milk and adding raw milk to the remainders. These production methods result in Mabisi of very diverse organoleptic properties and consistency (8).

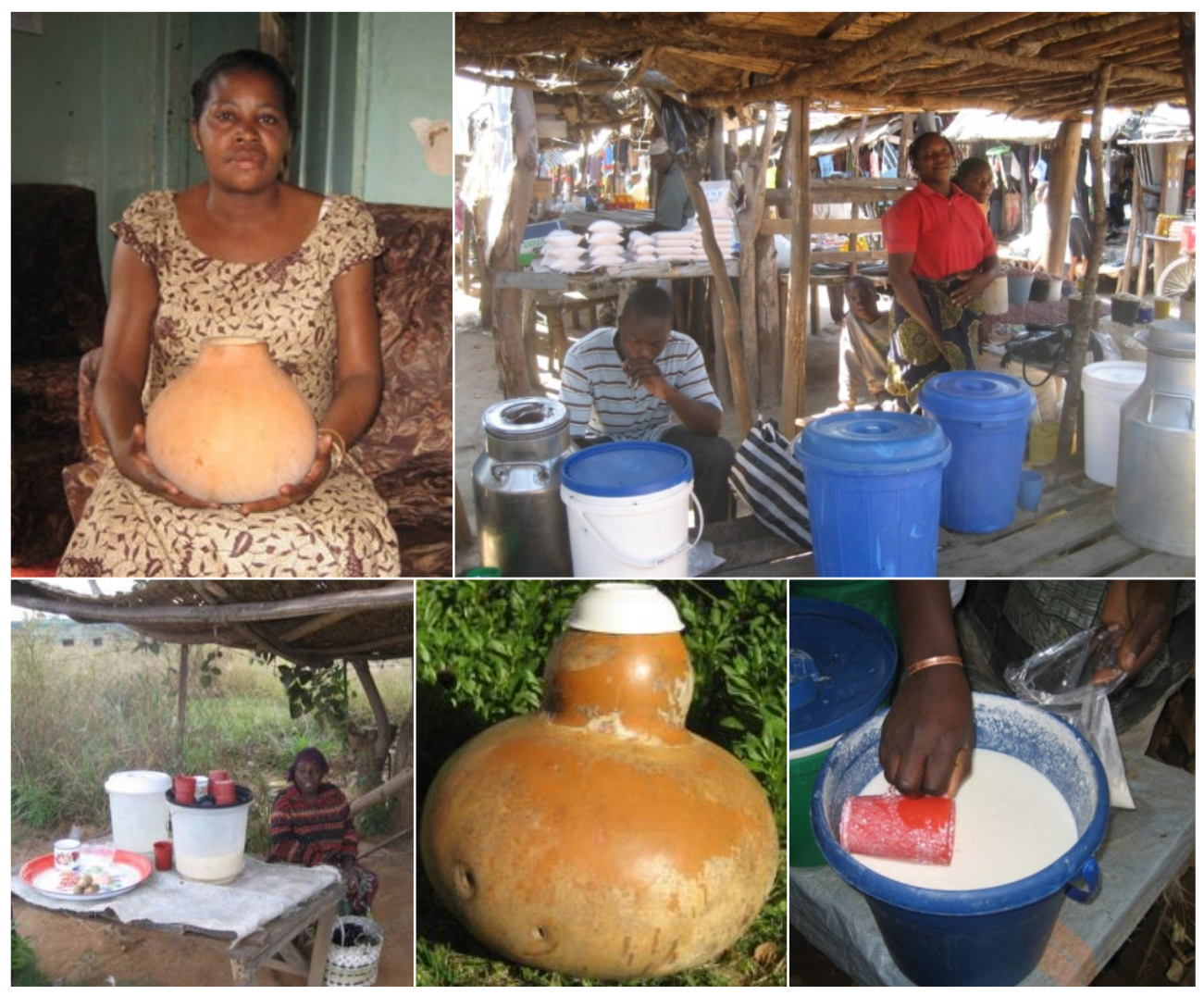

Figure 1.2 Mabisi producers who use different types of containers. Mabisi is produced at home and sold on the local market or along the main (national) roads. Mabisi produced in calabashes is sometimes transferred to plastic buckets for ease of transport and hygiene on the market. The calabash can then be used for another fermentation cycle. In other cases, Mabisi is produced and sold in plastic buckets or bottles and metal buckets. Pictures by Sijmen Schoustra. 


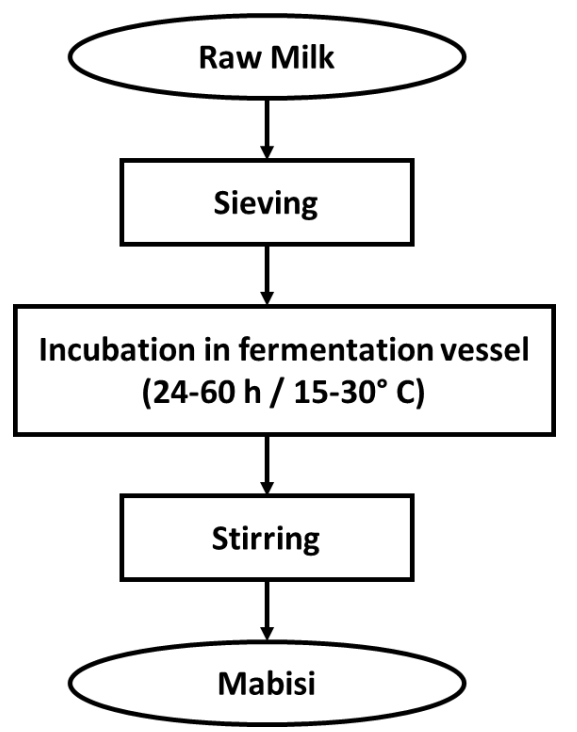

Figure 1.3 Basic production method of Mabisi. Many variations on this method exist (8). Picture adapted from Schoustra et al. (5).

Independent of production method, Mabisi is a widely consumed product throughout Zambia. It represents a tasty and nutritious product with a long shelf-life, but is also thought to have certain health benefits such as the prevention and cure of diarrhoea. Mabisi contains a mix of lactic acid bacteria in combination with other fermenting bacteria, which might possess probiotic properties $(5,9)$. Producers mostly consume their product with their family members at home and also sell their product on the local market to have an additional source of income. Increased production and consumption of Mabisi has important implications for nutrition security, public health as well as woman entrepreneurship, which are important aspects of the Sustainable Development Goals (SDGs) formulated by the United Nations. In Zambia, half of the produced raw cow milk goes to waste, while food insecurity and malnutrition persist at rural household level due to gender imbalances, differences in household involvement in agriculture and post-harvest losses caused by malfunctioning value chains of local products. Thus, converting raw milk into Mabisi, which is safe and nutritious, contributes to preventing food losses and promotes general health.

Increased production is not as straightforward as it sounds. Traditional Mabisi is produced by spontaneous fermentation, meaning that the milk is not pasteurised, and no bacterial starter culture is added to start the fermentation. As a result of this production method, Mabisi cannot be sold at formal markets under the national food safety law. A Mabisi-like product is currently on the market in urban areas, called LactoMabisi. For this Mabisi alternative, milk is pasteurised and a bacterial starter culture of cream cheese is added, resulting in an end product with organoleptic properties that differ from those of traditional Mabisi. Consequently it has a lower consumer preference than traditional Mabisi (10). Also, the health benefits of this products are not proven. To improve national health, traditional Mabisi should be consumed nationwide, also in urban areas. Producing Mabisi with pasteurised milk could yield a safer product than when raw milk is used. However, this would require a starter culture that closely resembles the characteristics of the original microbial community. The formulation of 
such a starter culture requires the establishment of the relation between product characteristics, quality and the microbial community in Mabisi. Moreover, the spontaneous fermentation of Mabisi might result in a safe and stable product that does not need to be banned from the official markets. To determine this, knowledge about the bacterial communities responsible for the spontaneous fermentation should be extended (5).

\section{Spontaneous fermentation}

Many fermented products are made with a defined starter culture, typically consisting of one or several microbial strains with known characteristics. Well-known examples include yoghurt, bread, and wine. Sometimes starter cultures are undefined and relatively complex, such as the starter culture for Gouda cheese (11). In contrast, products from spontaneous fermentation are not inoculated with a starter culture, but rather are fermented by whichever bacterial community is present in the raw material or residues on the fermentation equipment. This often results in a fermenting community with a high species diversity. Examples of Western products made through spontaneous fermentation are sauerkraut, sourdough, and parmesan cheese. In the latter case, 24 hours fermented whey is added to a new batch of raw cow milk before overnight fermentation (12). This method, in which an amount of finished product provides the starter culture for the fermentation of the new raw ingredients, is called back-slopping (13). In this thesis I distinguish two types of back-slopping: active backslopping, where a part of finished product is actively added to a new batch of raw material, and passive back-slopping, where the transfer of starter culture to the new raw material is not an active part of the production methods but rather "co-incidental"; via, for example, the surface of the fermentation vessel or the re-use of other equipment.

Back-slopping forms the (in many cases only) inoculum for the next fermentation and could be compared to the addition of a starter culture. There is however a difference between back-slopping and the addition of an undefined starter culture. Where in the case of Gouda-type cheeses the starter culture comes from the same frozen source, in back-slopping the source of the starter culture is the previous batch. This allows for the bacteria in this back-slopped community to adapt towards the environment over consecutive fermentation cycles. In other words, this allows for the community to evolve, depending on the selection pressures.

\section{Selection and domestication}

The driving force of adaptive evolution is natural selection (14). Natural selection works on the individual, where the individual with the largest reproduction advantage is selected. In a men-made environment, selection can also work on groups of organisms, where a group of species is artificially selected to provide desired characteristics. An example could be an apple orchard. Natural selection would favour the tallest tree that can catch most sunlight at the expense of other trees, and as a consequence produce most apples. When the apple farmer interferes, he or she might select a type of tree that produces many apples without overgrowing other trees. In that way the total yield of the orchard is maximised. 
Soon after the domestication of animals and plants, also microorganisms were domesticated. Although probably not as deliberate as was the case for animals and plants, bacteria that transformed raw materials into tasty, digestible, and safe products were selected. These wild-type bacteria, coming from variable and complex natural environments, found themselves in a relatively simple and nutritious environment, resulting in genomic specialisation, caused by a loss of genes unnecessary in the fermentation environment and acquisition of new traits for fermentation $(2,15,16)$. These evolved differences from wild-type bacteria are on an individual level, but the context of the microbial community in which these evolved strains are present, cannot be ignored.

\section{Co-evolution in spontaneously fermented Mabisi}

Co-evolution of microbial communities in a constant environment makes individual bacteria become specialists, which might lead to a stable and productive ecosystem (17-23). According to Gause's principle, microorganisms cannot live together unless they occupy their own niche, physically or chemically (24-26). Raw milk as fermentation medium contains many different fermentable resources, which form different niches (27). Besides chemical niches, a large calabash might have $\mathrm{pH}$, temperature, and dissolved oxygen gradients, which form physical niches. Other niches can also develop during fermentation due to cross-feeding and adaptive radiation $(28,29)$. Within one niche microorganisms compete against each other and the one with the highest fitness would eventually be fixed by natural selection (25). Niche specialisation also makes microorganisms less flexible to adapt to new environments and less able to fill in one of the other niches available in the environment $(28,30-32)$.

As all bacteria in the community have their niche and function, the community might be seen as a "super-organism". In that case this super-organism would be the entity of selection instead of a selection of individuals. In that way, a community would evolve as a whole to a certain environment. In the current convention in evolutionary biology, this is a rather controversial hypothesis as natural selection would not act at a group level (33). Although there are many who reject the idea of community selection, there are still indications that ecological communities might form integrated, novel evolutionary entities $(34,35)$. Whichever side one takes, including ecological aspects in the study of evolution seems the best way to investigate this controversy. In study set-ups fitness of individuals as well as their interactions need to be taken into account as there is constant interplay between the abundances of phenotypes.

The idea of experimentally looking into community evolution brings us back to Mabisi. The production method of Mabisi, where a calabash is used for consecutive fermentation rounds, allows for the bacteria, which are responsible for the spontaneous fermentation, to co-evolve. Due to the rough inner surface of the calabash, a residue of the previously produced Mabisi always remains to inoculate the next batch of raw material through passive back-slopping. According to local people, the fermented products used in this study have been produced for years or decades, by continuously propagating the product using the same calabash containing a starter culture. From this practice, we can assume that the microbial communities in those products have reached an evolutionary equilibrium, representing end-points of long-term co- 
evolution. Therefore, the microorganisms present in these communities are expected to show high levels of co-operation. On the contrary, in case clean buckets or new calabashes are used, all microorganisms present originate from the raw milk or the environment. Most likely they are only recently brought together at the moment Mabisi preparation is finished, in about 48 hours. It is therefore unlikely that these microorganisms have reached an evolutionary equilibrium and thus the levels of finetuned interactions in such communities are expected to be lower than in long-standing calabash communities. This difference in level of co-evolution and interactions in the ecosystems might lead to different responses of these communities to disturbances, such as bacterial invasions or changing environments. A co-evolved community, present in calabashes, might therefore produce a more standardised Mabisi with preferred characteristics.

\section{Experimental evolution}

For many years the study of evolution was based on the sampling of variation present in nature and by looking back to reconstruct how that variation arose. Many natural systems have provided key insights in evolutionary processes such as adaptive radiation (36-39). However, such natural systems can only be used to study the effect of changes in environment or interactions when they occur naturally, initiated by chance and without much replication. Another challenge is that multicellular eukaryotic organisms in general have generation times of weeks to years, making prospective laboratory evolutionary studies unfeasible. As a result, most research is based on fossils or would take several generations of researchers to have a glimpse of the whole picture of evolution. To study the reproducibility and predictability of evolution, we need to observe evolution in action by replicating experiments in set environments (40). We can do this by putting replicates of organisms in a common garden experiment allowing long-term reproduction and studying the adaptations we see. This is called experimental evolution $(6,41)$. Microorganisms are an ideal model system for experimental evolution, as they are small, have short generation times and can survive freezing and defrosting. An example is the experiment of Bachmann et al. (42), where a plant-derived Lactococcus lactis strain was brought in a dairy environment to adapt in the same way as Lactococcus lactis strains found in fermented products.

The research area of experimental evolution is shifting from experiments with a welldefined single species to those using simple community reconstructions and complex microbial communities. In this way ecological interactions are included in evolution experiments $(2,43-45)$. Adding an aspect of time to ecology brings the findings closer to what we see happening in nature compared to looking at evolution or ecology alone. These studies towards the interactions between ecology and evolution, the ecoevolutionary dynamics (46), ask for model systems that are simpler than natural systems while they still contain the dynamics of a complex system. In that way the complete community structures, including all players and interactions, can be analysed.

Most factors affecting the direction of evolution (such as environmental conditions, interactions with other organisms and availability of genes and mutations) are difficult to disentangle in experimental setups. Not the least because the activity of one 
organism can change the environment of co-occurring organisms. The ecology and evolution of one particular organism is often studied on ecological islands (such as pieces of woodland fragments surrounded by agricultural land, lakes or actual islands in seas or rivers) because they offer a confined semi-closed system and simplified context (47). The communities found in Mabisi provide another ecological island. They form a unique model system for eco-evolutionary dynamics for two reasons. First, the microorganisms present in Mabisi form a stable community, which most likely had years of co-evolution in a natural environment. The evolutionary mechanisms found in these communities are therefore hypothesised to be representative for those present in more complex systems (48). Just as in many other bacterial communities, the natural community of Mabisi is diverse. However, it is not as diverse as bacterial communities in the soil or the human gut, which would be too hard to disentangle with current methods. Second, products are not shared between producers and production methods differ slightly, resulting in somewhat different types of Mabisi from producer to producer, which is probably linked to differences between the bacterial communities in the respective Mabisi types. The various microbial communities had the chance to co-evolve in isolation and are therefore potentially different whilst still having the same function, i.e. the fermentation of Mabisi.

\section{Starter culture}

The microbial ecology and dynamics of the fermentation and their relation to the characteristics of the resulting food need to be fully understood to allow process optimization and the formulation of starter cultures that result in the nutritionally best and most appreciated foods (49-52). My thesis sheds a light on the long-term dynamics that can be expected in a traditional starter culture. Also, the feedback loops during fermentation, where one species can only grow after another one has produced an essential metabolite are an important aspect for starter culture formulation. After gaining full understanding of the properties of the natural Mabisi community a Mabisi starter community can be formulated.

Other spontaneously fermented products might have similar beneficial characteristics. In this study we also look at Lait caillé, another dairy product from spontaneous fermentation originating from Senegal. Lait caillé is prepared using cooked cow milk or milk powder, which are assumed to contain less bacteria than the raw milk used for Mabisi preparation. A wooden bowl is used as fermentation vessel, which might have functions similar to the calabash, such as in providing the starter culture. These differences and similarities in production methods can have an influence on the microbial communities in these products which can give valuable insights in community dynamics. Besides being an interesting model system, both Mabisi and Lait caille have important implications for local producers, giving an added value to study. Suggestions on how results described in this thesis can be used for development can be found in the subchapter 'Science for development' in the general discussion. 


\section{Aim of the thesis}

The aims of this thesis are 1 ) to provide the first step towards the use of microbial communities of Mabisi as model system for studies of eco-evolutionary dynamics, and 2) to provide ecological and evolutionary insights in Mabisi fermentations as part of a research for development project.

The first aim has a threefold set-up of experiments with an increasing level of control, while complexity is decreasing (Figure 1.4). In the first part Mabisi was produced in traditional ways in the field, which resulted in a low level of control of variables. For the second part, I brought the microbial communities to the lab and used controlled environments without changing the diversity of the natural communities. The third part consisted of using simplified natural communities by decreasing diversity through serial dilutions or reconstructing natural communities with isolated species into synthetic communities $(53,54)$. These synthetic communities were subjected to the same disturbances as the natural communities to analyse factors that influence natural community dynamics. These three parts cover the steps in translating results of single strain evolution experiments to observations in natural communities, all while using only one model system.

The second aim focusses on the practical application of bacterial dynamics for dairy fermentation. This aim is entwined in my entire thesis. When constructing and interpreting the studies performed, the use of the outcome for producers and consumers of spontaneously fermented foods was taken into account. A general conclusion on the use of these findings is presented in the final chapter of the thesis.

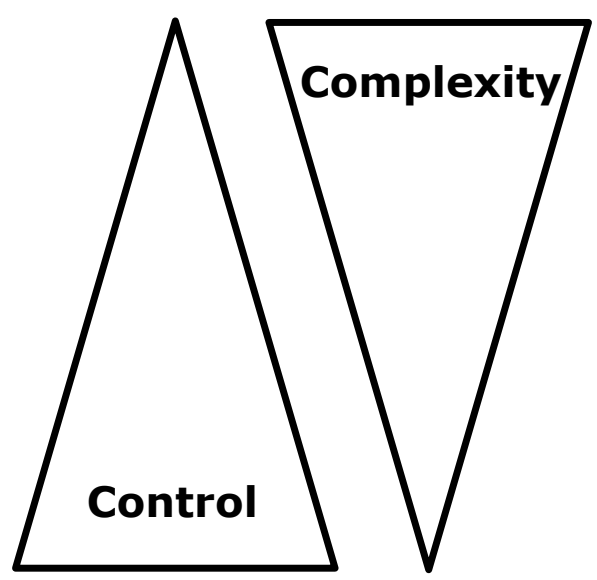

(Complex natural systems)

1) Traditional fermentation

2) Natural communities in the lab

3) Synthetic ecosystems

(Single strain experiments)

Figure 1.4 Set-up of experiments to cover translation between single strain experiments and complex natural systems. 


\section{Outline of the thesis}

In Chapter 2 I explain the use of fermented products in evolution experiments. I discuss which evolutionary theories concerning community evolution could be addressed with the diverse microbial communities found in most spontaneously fermented products. In the past years the model systems have been mostly simple systems of one or only a few interacting organisms. In this chapter I explain how more diverse systems add to our current understanding of evolution and in the rest of my thesis I make a start in using microbial communities of Mabisi as such a model system.

Apart from being the model system of this thesis, Mabisi is primarily a local product made by traditional fermentation and produced in rural areas of Zambia. The first step of my research was therefore to travel to these rural areas of Zambia to see how Mabisi is produced and to analyse the bacterial communities that are responsible for the fermentation. In Chapter 3 I explain a method that we developed using simple filter paper disks to stabilise bacterial communities sampled at all stages of fermentation for transport and later analyses. I used this method to analyse the bacterial communities when different production practices were employed for making Mabisi (Chapter 4). The effect of fermentation vessel on the final microbial community was investigated as more and more people use plastic containers and buckets for the fermentation instead of calabashes. Also, the effect of back-slopping was assessed, as this would be an easy way of stabilising the microbial community that is responsible for the fermentation and could counter the possible loss of flavour from fermenting in plastic containers.

In further experiments in my thesis I took the diverse microbial communities from fermented products into the laboratory to have more control over variables. In Chapter 5 I show results of an experiment where I brought six different microbial communities from Mabisi in a new, common environment in the laboratory and propagated these in milk for two months. We compared bacterial species composition at the start and the end of the experiment. To analyse what factors influence species composition, five scenarios with a hypothetical species composition were modelled. The observed and modelled species composition were compared to measure the influence of the factors on changes in species composition in this new environment.

The last chapter of my thesis, Chapter 6, focuses on another spontaneously fermented milk product called Lait caillé, which originates from Senegal. We analysed the microbial communities in this traditionally produced product and added two commercial strains from a probiotic starter culture. The research objective here was also two-fold, besides the interest in the bacterial stability of the microbial community (over time and upon invasion of an alien microbe), this study also showed the possibility of enhancing this widely consumed product with probiotic properties. Besides the introduction of a probiotic strain, also the possibility for a pathogen to become a member of the community in a fermented product has important implications for food safety as well as community stability. Some preliminary results concerning pathogen invasion in Mabisi are shared in the General Discussion. Here I also explain how the results of all these experiments add to our knowledge of community evolution as well as the possibilities of science for development. I further outline potential future work using synthetic communities, on which some preliminary experiments have been performed. 
Chapter 2. 


\section{Microbial communities from spontaneously fermented foods as model system to study eco-evolutionary dynamics.}

\section{[Perspective]}

Anneloes E Groenenboom ${ }^{a, b}$

Eddy $\mathbf{J}^{\text {Smid }}{ }^{\mathrm{b}}$

Sijmen E Schoustra ${ }^{a, c}$

a Laboratory of Genetics, Wageningen University and Research, Wageningen, The Netherlands

b Laboratory of Food Microbiology, Wageningen University and Research, Wageningen, The Netherlands

c Department of Food Science and Nutrition, School of Agricultural Sciences, University of Zambia, Lusaka, Zambia. 


\section{Abstract}

Evolutionary forces are widely recognised as the key drivers of patterns of biodiversity. This has resulted in a large body of theory, some of which has been tested experimentally by mimicking evolutionary processes in the laboratory. In the first part of this perspective we outline what model systems are used for experimental testing of evolutionary theory, ranging from simple microbial communities in the laboratory and, more recently, to complex (natural) communities. Microbial communities of spontaneously fermented foods are a very suitable model system to study ecoevolutionary dynamics. It is a model system that combines the complexity of a natural community with the ease of analyses of a synthetic defined community. It therefore gives the ability to investigate specific species in a natural community without becoming too challenging. Due to rapidly developing sequencing techniques, the diversity in these communities can be analysed with relative ease while hypotheses developed in simple systems can be tested. In the second part we explain which research questions with an evolutionary background can be addressed using these microbial communities from fermented foods. We discuss species frequency in space and time, the diversity-stability relationship, niche space, fluctuating environment and community coalescence. Hypotheses of the influence of these factors on community evolution are given as well as a short indication of the experimental set-up of such studies when microbial communities of spontaneously fermented food are used.

\section{Introduction and scope}

Selective pressures are an important factor in shaping biodiversity in ecosystems. Patterns of biodiversity can be described in terms of the number of species present and the distribution of individuals over these species. Due to selection pressures from the environment these patterns can change, analogous to selection acting on populations with standing genetic variation. In this way, changes in biodiversity patterns due to (environmental) selection can be regarded as an evolutionary process and referred to as eco-evolutionary dynamics (46). Research into understanding how these evolutionary forces shape and maintain this diversity has been mainly comparative and retroactive; reconstructing the path of evolution by observing and interpreting current (evolved) states of communities. Experimental approaches to study evolutionary processes have been developed relatively recently using techniques of high throughput DNA amplicon sequencing. Since experimental tracking of communities is challenging, thus far, most experimental evolutionary research is performed using highly simplified systems, mainly (micro-) organisms with a short generation time. Evolutionary research on a complex natural community can be challenging, due to a large diversity of organisms in different trophic levels and their interactions. As a result, one or only a few organisms or genotypes are used for longterm propagation experiments without considering possible interactions with other organisms and with the natural environment. Experiments using complete communities from a natural environment could provide insights in interactions and their long-term eco-evolutionary dynamics occurring in nature. In many fields of biology, the concept that "everything is connected" is extensively discussed and studied, resulting in models of metabolic networks, genetic regulatory networks and trophic structures. Currently, evolutionary research rarely links with ecology by 
including experiments addressing the consequences for ecological dynamics let alone the evolutionary impact of this connectivity, mainly due to technical difficulties. Understanding the influence of evolution on co-existing organisms could deepen our ecological understanding of community performance.

To advance, natural model systems are required with a limited number of species and interactions. A few of these have been reported, such as microbial communities in tree holes and synthetic communities derived from marine environments (55-57). Traditional fermented foods, containing mixed communities of fermenting microbes, add to such natural tractable systems comprising of micro-organisms, making them suitable for experimental ecological and evolutionary research (58). In this perspective, we elaborate on the types of (eco-)evolutionary questions that may be addressed using microbial communities from spontaneously fermented food products or other model systems. We will show how community evolution has been studied so far and what experimental model systems were used. Further, we will elaborate on the added value of using microbial communities from nature. Last, we will show how these model systems can add to our understanding of community performance by showing what evolutionary theories can be tested with the model. In the boxes we provide additional information about experimental evolution as well as a short outlook on possibilities for research applications.

\section{Microbial model systems}

In this section we will explain various model systems of increasing complexity that are used for evolution experiments.

\section{Single strain evolution experiments}

Already in the $19^{\text {th }}$ century Dallinger started controlled evolution experiments with microbes - or at least invisible organisms (Box 1). A large scale follow up of this new way of studying evolution came much later (59-62). Microorganisms are of interest to evolutionary biologists because they are small, have short reproduction times and can easily be stored and preserved for long periods of time (63). The short generation time of microorganisms allows us to see evolution in action and even attempt to predict evolution (64). The ability to store bacterial cultures by freezing and later thawing them without loss of viability allows for direct competition experiments between evolved and ancestral types (63). Due to the these advantages microorganisms already mitigated a lot of challenges of evolutionary research. Most experiments focus on the evolution of a single bacterial strain in a defined laboratory environment $(65,66)$, allowing for direct tests of specific variables on the outcome of evolutionary processes. This includes the dynamics of adaptation to novel environments and stress conditions such as the presence of antibiotics, patterns of repeatability of evolution, predictability of evolution through the mapping of evolutionary pathways on adaptive landscapes, and details and constraints of evolution at the genome level $(41,62,63,67)$.

The approach of experimental evolution using microbes could be expanded to the community level. as most naturally occurring microorganisms live in close proximity to hundreds or even thousands of other bacterial species and organisms from other taxa. However, analyses of most natural environments would result in large quantities of 
data, which might make the formulation on predictions for evolutionary experiments difficult. As a solution and to simplify these microbial communities, they can be shaped into synthetic communities with only a limited number of focal (micro-) organisms.

\section{Synthetic communities}

Naturally co-occurring microorganisms can be isolated from their environment and brought together in the lab in pre-determined concentrations. These so-called synthetic communities can be used for studying evolutionary processes under strictly defined conditions $(43,68-72)$. Due to previous interactions and co-evolution these bacteria are more likely to represent essential parts a natural community compared to combinations of laboratory strains which have no historical connection $(53,73)$. In this way, synthetic communities are assumed to represent nature more accurately than most artificial communities while keeping the simplicity that is needed for experiments. A very elegant synthetic community was constructed recently by combining 33 strains from a range of environmental sources (74). Upon long-term propagation through serial transfer of 48 days in a laboratory environment, around half of the strains were maintained in the community that could be analysed for a range of functional properties (74).

Using synthetic communities also poses two challenges. First of all, researchers face the difficulty of isolating the bacteria that are the key players in the community. Some community members might not grow on culture media in the laboratory and will therefore be excluded from the community of isolates. Other bacteria that were isolated might not have been a member of the natural community but were incidentally present. The second challenge is to achieve the relevant or representative degree of diversity. A very simple model will not represent nature accurately. Species (initially) present at low abundance may be important for eco-system functionality but may be missed when assembling the synthetic community. In an experiment using a microbial community from cheese, an initially very rare species became predominant upon longterm propagation in a novel environment (3). A further illustration is an experiment on the influence of bacteriophages on culture diversity of Spus and his colleagues (2015). They found that the simple bacterial blends used in their experiments did not represent the diversity of the original complex starter enough to evaluate and grade the role of phage predation (11). Later, Spus repeated the experiment with more complex blends of bacterial strains which showed the influence of phage predation on community diversity (3).

Although the approach of extracting strains from natural communities into synthetic is very valuable, these two challenges might make the translation into 'real world' ecology and evolution unrealistic (75). This motivated the search for a better model system. What we need to find is small confined 'islands' of microorganisms in which the number of players and their interactions is limited and therefore manageable. In these 'islands' no selection or extraction of species into synthetic communities is required for communities to be experimentally tractable.

Bell and colleagues found these 'islands' in the form of small pools formed by the roots of beech trees $(17,76)$. As the number of players in the pools on beech tree roots is limited, it is not necessary to extract players and put them together in a set frequency. 
The natural communities can directly be used for experiments. Also, the communities can be rebuild by using isolated strains for experiments with a lower diversity. Consequently, all the steps between single strain behaviour towards the behaviour in natural systems can be compared. The risk of losing vital interactions will be low and observations in the lab could represent nature.

\section{Fermented foods as model systems}

Traditionally fermented products can form another 'island-group' of interest $(5,54,77,78)$. Many traditional fermented products rely on spontaneous fermentation, which means that they have little human interference as they are not produced using defined starter cultures, but are fermented by a naturally available microbial community. These natural communities are usually diverse but not too complex (e.g. up to 13 main players and numerous other species at very low abundance in three traditional fermented products from Zambia (5)).

In order to improve organoleptic properties of these products, producers often re-use a finished fermented product for the production of a next batch of the same product (79), in this way propagating the microbial community that underlies fermentation. In the food science domain this process is referred to as back-slopping (13). Backslopping can also occur passively, by the re-use of non-sterilised fermentation equipment, like previously used vessels. These vessels will become the long-term habitat of the fermenting microorganisms. In other production methods back-slopping is done actively, like for the production of Illa-type Mabisi, a fermented milk product from Zambia, as well as for parmesan cheese production $(12,80)$. The so called natural whey starters which are used for the production of Parmigiano Reggiano (parmesan cheese) consist of bacteria which have co-existed for long periods of time with enough nutrients available to go though many generations. This method is intended for the production of a stable quality product, but can be compared with a standard evolution experiment (as explained in Box 2). The diverse microbial interactions are therefore assumed to be more like those of an evolved community.

Many traditional fermented products are dominated by communities of lactic acid bacteria (81-84). Also other microorganisms such as acetic acid bacteria and yeasts and moulds can play important role in fermented foods. The physiology, metabolism and genetics of lactic acid bacteria have been studied in great detail because of their dominant role (85-87). The extended knowledge of metabolite production and growth profiles of these bacteria, can help in understanding the observed evolutionary pathways. Due to an ongoing development in sequencing techniques the methods of analysing these communities are becoming more available and affordable. This now makes it feasible to characterize large numbers of communities required for analyses of evolutionary outcomes of replicated evolution experiments.

Using food products as a model system stimulates collaboration between fundamental research groups focussing on evolution and research groups working in the field of food sciences and applied microbiology. This multidisciplinary approach is expected to lead to fermented food with improved properties. An example of this type of research can be found in Box 3. 
There are many fermented food products that are produced spontaneously and therefore contain a diverse microbial community. Not all of them will be suitable for evolution experiments. In our opinion fermented liquids, such as milk, with communities containing bacteria will be most convenient concerning experimental setup and culture or DNA based downstream processing. Natural communities might be even better represented by communities including mounds and yeast. However, an increase of diversity will also increase complexity of sequencing and analyses.

In summary, the microbial communities present in spontaneously fermented products make a very useful and interesting model system for evolutionary research, complementing the few other model systems that have been developed $(56,76,88,89)$. Three aspects contribute to this; 1 ) their limited complexity that still represents nature, 2 ) the production methods allows for the communities to adapt to their environment, and 3 ) the available knowledge about the individual players in the community. In the next section we will explain some concepts concerning community evolution that can be addressed using microbial communities.

\section{Study eco-evolutionary dynamics with microbial communities from fermented foods}

The suitability of a model system depends on the experimental design for testing hypotheses concerning changes in patterns of biodiversity due to selective forces. This process can be described as species sorting (90) or as eco-evolutionary dynamics, which can also be more formally defined as "interactions between ecology and evolution that play out on contemporary time scales, with "contemporary" intended to encompass time scales on the order of one to hundreds of generations" (46). In this way, ecological changes can lead to short-term evolution, and changes in patterns of biodiversity can be viewed as an evolutionary process - in line with changes in allele frequency in populations with standing genetic variation in response to selection $(91,92)$. Various theories concerning eco-evolutionary dynamics of communities have developed (4,93-96). Here we will highlight some theories that could be addressed in experiments using microbial communities from natural systems such as fermented products. For these theories we will also indicate how the experimental setup could look like. Figure 2.1 indicates how much the different model systems mentioned in section 2 would represent nature and how easy it is to study individual species and community structure over time. The figure also shows how well the different evolutionary questions and theories mentioned in this section can be addressed using these model systems. Table 2.1 lists (dis)advantages of these different model systems for answering the different evolutionary questions. 


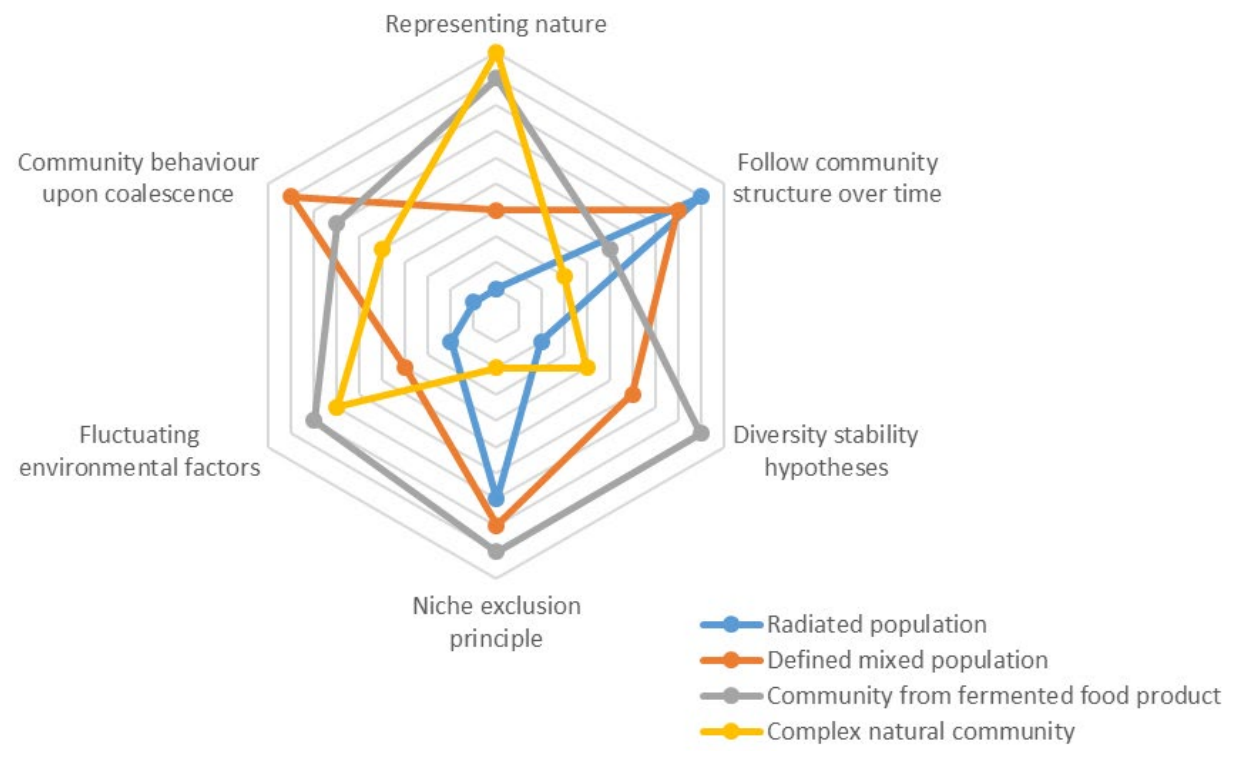

Figure 2.1 Different model systems and their applicability for study of evolutionary theory. Values are a combined value for representability of nature and the ease of using the model systems in the experimental setup. For now more complex communities have the disadvantage of more complicated analyses, where not all players can be isolated and studied individually. When isolation and typing techniques will become more available and affordable due to developments in the field of sequencing, the applicability of more complex communities as model system will increase.

\section{Patterns of diversity}

In natural communities the frequency of various species varies in space and time. In these communities, variations in the patterns of species abundance in space and time can be measured and differences in these patterns can be linked to potential causal factors $(88,97)$. A study focussing on baker's yeast revealed that analyses of these eco-evolutionary dynamics can be done by sampling microbial communities in the same food product or type of product, but derived from in different geographical regions and over time. The different environments and slight differences in production methods of the fermented foods are variations in selection pressures that shape the microbial community structure (88). The differences and similarities found can be linked with environmental data, in order to extract patterns caused by environmental factors. This has also been inspirational to modelling studies focussing on traits and that explored in what way ecological factors and natural selection can influence community structure of multiple coevolving species. Taken together, observations of different patterns will allow us to generate hypotheses and predictions that are testable using experimental communities.

Testing of predictions on what factors have the biggest influence on community diversification, can be done by challenging the microbial communities in the laboratory 
in a selection experiment. These factors can be related to the degree of diversity of the community, the number of niches that are available in the environment as well as the evolving interactions within the community. All these aspects apply to microbial communities in spontaneously fermented foods and other natural microbial communities.

\section{Diversity stability hypothesis}

The biodiversity-stability hypothesis poses that a more diverse system has a higher stability in terms of functionality (98). The functionality of microbial communities in fermented food products is based on their ability to convert the available nutrients in the food matrix into metabolites to obtain the required product characteristics. The clear definition of functionality allows for an easy assessment of the loss or change of functionality, e.g. unsuccessful acidification, reduced breakdown of proteins or offflavour production. A higher species diversity within the microbial community can result a stable functionality of the community due to a back-up function $(17,68,99)$. If, for various reasons, certain members of the community are not functional anymore, a diverse community might contain members that can take over the lost function. A higher diversity also causes a lower number of unoccupied niches, due to for example unused nutrients (100). In that case those niches are not available for any invader, which makes the whole system more likely to keep its functionality and not be destabilised by a non-co-operator, like a food spoiling or pathogenic microorganism (69).

Whether a natural community is more stable when it is more diverse can be tested by manipulating these natural communities to become less diverse. During propagation in an evolution experiment, a fraction of the communities is periodically transferred to fresh medium (Box 2). By using sequential propagation with an extremely high dilution factor of the inoculum, only those bacteria present in the highest numbers will remain, which strongly decreases the diversity in the community. Whether the diversity which is lost was crucial can be tested by studying the change in fermented end-product characteristics and testing the resilience of the microbial community against stress or invaders. Also the influence of diversity on evolution of the community itself can be tested (76).

Sometimes predators can facilitate stability. These non-co-operators can stabilise a diverse community when growth rates of different members greatly differ. The member with the highest growth rate is the preferred victim of a predator according to the kill the winner principle $(3,101)$. The fastest grower in a community could potentially provide most nutrients for a predator and will therefore be the preferred pray, keeping the community stable. This phenomenon is closely related to "negative frequency dependent selection" of the focal strains, where an increase in frequency of an organism has a negative effect on the fitness of that organism $(41,102,103)$. Apart from predation, this negative frequency dependent selection can also be caused by various other limiting forces, like food resources, cross-feeding or physical space. The magnitude of the influence of these forces can be studied by reconstructing natural bacterial communities using frequencies that differ from the frequency found in nature. The speed in which these communities will return to their original frequencies, if they do, can give indication of the strength of these forces. 


\section{Niche space}

Over the years various hypotheses have been developed concerning niches in established communities. One of the oldest hypotheses concerning niches is the niche exclusion principle which states that one niche can only be occupied by one organism (Gause 1934; Hardin 1960). If two species occupy the exact same niche, descendants of the most fit organism will gradually take over from the descendants of the less fit organism.

In natural environments the niche is defined by various depletable resources, like nutrients and space, and non-depletable resources, like temperature and $\mathrm{pH}$, which together form a multidimensional niche space (Hutchinson 1957). Because of these different dimensions, in theory, organisms can live together in a community as long as one dimension in the niche space is not overlapping between the two organisms (107109). The magnitude of the overlap of niches, determines the level of competition between the species. This allows for many different organisms in a natural community as there are many different niches available.

Free niches might increase the chance of invasion by an alien species $(100,110,111)$. The availability of unoccupied niches may result in character displacement (112) and adaptive radiation (113), where organisms adapt to occupy other niches. A classic example of microbial adaptive radiation is the work on Pseudomonas fluorescens in a static environment, where initial mono-cultures diversify into at least three phenotypically different types that each specialized on a specific niche that is defined by an oxygen gradient (113). Without external fluctuations the amount of niches will remain stable and should be equal to the amount of species present in the community. By propagating a microbial community, like a microbial community derived from a fermented food, with different numbers of species or by adding or removing niches it can be tested whether these two are indeed so strongly linked.

\section{Fluctuating environmental factors}

In natural communities we cannot neglect external fluctuations. The continuously changing environmental factors in batch culturing are to a certain extent comparable to fluctuations that can be found in natural ecosystems, like seasons and tides. Under such dynamic conditions, nutrient rich periods are alternated with nutrient poor periods. These fluctuation give rise to the possibility of different organisms to flourish in different moments of time, resulting in a more diverse community. The could be a trade-off between growth rate in the exponential phase of fermentation and survival rate in the stationary phase that is following the fermentation which will result in a balance of organisms that have a different strategy $(114,115)$. While predicted in theory, these microbial experiments have demonstrated that such a trade-off exists.

The influence of these fluctuations on community structure can be investigated by varying the time regime of the batch fermentations that is also characterized by phases of rapid population expansion followed by periods of stationary phase at the species community level. In this way, the balance between fast growers and those with high survival could change. It is possibly very difficult to completely eliminate some players in the community (3), but it is hypothesised that when there is no stationary phase, 
the community will mainly consist of fast growers, while at constant low nutrient levels the community will consist of mainly slow growing survivors (116).

\section{Community coalescence}

Another natural phenomenon that can be studied using bacterial communities is the effect of coalescence of communities. The term "community coalescence" was introduced recently (117) and describes situations were entire communities interact because the environments they are in are physically mixed sparking this interaction. In nature we see this for example during soil tillage and flooding events, but also while eating and kissing. The performance of single species do not always give a good indication of how the species will behave in a community (118). The coalesced community might be a combination of the two initial communities or be dominated by either of them, dependent on the best performing combination (119). The influence of co-evolution in the outcome of community coalescence can be investigated using microbial communities from fermented products. Combining two or more co-evolved communities can provide information on how specialised the evolved interactions within the communities are. Besides this coalescence of similar communities, also the mixing of a fermented community with the community of a raw product has important implications for the formation of the fermented product. Besides studying the influence of co-evolution on the outcome of community coalescence, also the evolutionary results of regular coalescence occasions can be studied using fermented products. $A$ further interesting avenue here is the presumed beneficial interaction between microbial communities from fermented foods and the gut microbiota (120). The interactions between these communities has presumed beneficial effects that warrant further study, for instance by adding spontaneously fermented foods into simulated gut systems (121).

\section{Conclusion}

Microbial communities of spontaneously fermented foods present a promising model system to experimentally test evolutionary theory on eco-evolutionary dynamics, such as the effect of selection pressures on changes in patterns of biodiversity. This would complement to other experimental systems that have been developed recently and would bridge work on synthetic and natural communities. Microbial communities like the ones from spontaneously fermented foods bear several intrinsic advantages for executing evolution experiments: short generation times, small size and ability to be stored frozen and defrosted to perform competition experiments (fitness tests) between evolved and ancestral lines. Moreover, these natural microbial communities have a limited number of players and form an island of microorganisms that does not have a lot of influx from the outside the confined system boundaries. These communities also have a clear function which makes it easy to follow up on evolutionary changes. All these characteristics make bacterial communities from fermented food products an interesting model system to test long standing theories in community ecology and evolution and increase our understanding of evolution and its drivers. 


\section{BOX 1 A short history of evolution experiments}

William Dallinger was the first to report about experiments testing evolutionary principles (122). By slowly increasing the temperature of the environment of microorganisms, he allowed these microorganisms to adapt to a temperature at which they would normally never grow. When returning them to their old environment he concluded that this adaptation came with the costs of growing slower in their old environment. His results show the principle of adaptation and trade-offs.

The approach of Dallinger remains in use today by evolutionary biologists. In the last two decades a lot of evolutionary concepts have been studied using mainly single organisms in controlled laboratory environments. It shows that even very simple laboratory model systems are very useful in addressing numerous fundamental questions on the dynamics of evolution. The longest ongoing evolution experiment is the setup started by Richard Lenski. In 1988 he started transferring 12 lineages of Escherichia coli on a daily basis in minimal medium. He used these lines to study adaptation and diversification, trade-offs, consequences of mutators and the influence of population size on drift among various other theories in evolution (Elena \& Lenski 2003; Rozen \& Lenski 2000; Lenski \& Travisano 1994; Lenski 2017; Deatherage et al. 2017; Sachs \& Hollowell 2012 etc.).

\section{BOX 2 Set-up of evolution experiments}

The set-up of evolution experiments has several general principles. Organisms are transferred to a set environment and are sequentially transferred to a fresh but similar environment at regular time intervals. During these transfers the organisms go through several generations, the number of which is determined by the dilution factor and the nutrients available. By taking samples after a particular number of transfers, changes in fitness and population composition can be monitored. A typical classic evolution experiment is performed with a single species which is allowed to evolve for a long period of time - i.e. hundreds or thousands of growth cycles/generations. In this way, beneficial mutations can deliver fitness advantage to variants. These advantages can be measured as increased relative abundance of the organisms carrying the beneficial mutation.

The experiments can be set-up with different variables; in starting genotypes, environment and ways of transfer. The type and number of bacteria that are transferred to the next cycle has great influence on the outcome of the results (127). Since mutations often occur random over the genome (with the exception of hotspots) only with a considerate number of replicates it is possible to draw conclusions to an observation.

By the production of spontaneously fermented foods using some material of an old batch to initiate a new batch ("back-slopping" (13)), the production method resembles an evolution experiment. The microbial community that ferments the raw ingredients are transferred to a new environment which is rich in nutrients so they can undergo many generations.

Consistency in production practices gives a stable environment for the community causes the community to get close to an evolutionary endpoint and all individuals to 
reach a fitness peak. The production method of Parmesan cheese is a clear example where backslopping can cause the microbial community to stabilise and in that way stabilise product quality. In case of mimicking the spontaneous fermentation in a preused container, the bacteria that attach themselves to the wall it might be necessary to transfer over the planktonic ones. Analysing these co-evolved communities can give insight in environmental factors shaping microbial communities.

In practical terms, microbial communities from spontaneously fermented foods can be used as follows. Product samples can be obtained from local producers of a given fermented product. Microbial profiles in these products can be obtained through various methods, such as classical culturing, or DNA based techniques of clone libraries and $16 \mathrm{~S}$ amplicon sequencing $(5,128)$. Product samples can be brought to the laboratory and used to found laboratory communities, for instance by inoculating a fraction ( $1 \%$ volume) of a finished product to fresh raw material (such as fresh milk). The same product sample can be used to found replicate populations or lineages. These newly founded laboratory communities can be allowed to grow to carrying capacity of the growth medium and then transferred to fresh raw material. Through this process of serial transfer, the long-term dynamics in biodiversity patterns can be studied, analogous to classical experimental evolution. Performance of the community can be studied for instance by measuring metabolic output in terms of aroma profiles using GC-MS or HPLC (129). This metabolic output can be linked to species composition and changes in metabolic output due to changes in species composition can be monitored.

\section{BOX $3 \quad$ Evolutionary applications}

We have only just started to explore potential of spontaneously fermented foods as experimental system. Apart from giving fundamental insight in microbial community dynamics and experimentally scrutinizing aspects of the evolution theory, the outcomes of research using microbial communities from fermented products will help understanding multiple-strain fermentations and how to manipulate these processes to obtain high quality fermented food products.

For local African communities, the large scale production of the traditional fermented products can be of crucial economic importance. Due to current regulations, local producers are sometimes not allowed to sell non-pasteurised products on the market. However, the pasteurisation of the raw milk will not only kill of pathogens, also the naturally present fermentation inoculum will die, leaving no starter for spontaneous fermentation. Providing a stable starter culture for these fermented products can be a great nutritional and economic importance for the local communities. So far, most bacterial starter cultures contain only one or two bacterial strains. In order to represent the original product accurately, the starter culture of the traditional fermented food might need more complexity. An understanding of community interactions and coevolution is required. Results of the types of research mentioned in this article might therefore be of great importance. When using strains from spontaneous fermentation at an industrial scale, international regulations on the protection of biodiversity (Nagoya protocol) need to be adhered to in order to protect interests of traditional producers (130). 
Also products currently produced on industrial scale could be improved when more is known about complex starter culture dynamics. The problems industries have to deal with range from contamination by pathogens, to frequency changes, plasmid loss, phage predation and mutations. In the industrial production of the probiotic lactic acid bacterium Lactobacillus rhamnosus, a mutation caused this bacteria to lose its flagella, which was crucial for its probiotic activity (131). By increasing our understanding of these problems, we might decrease their occurrence in industry. 


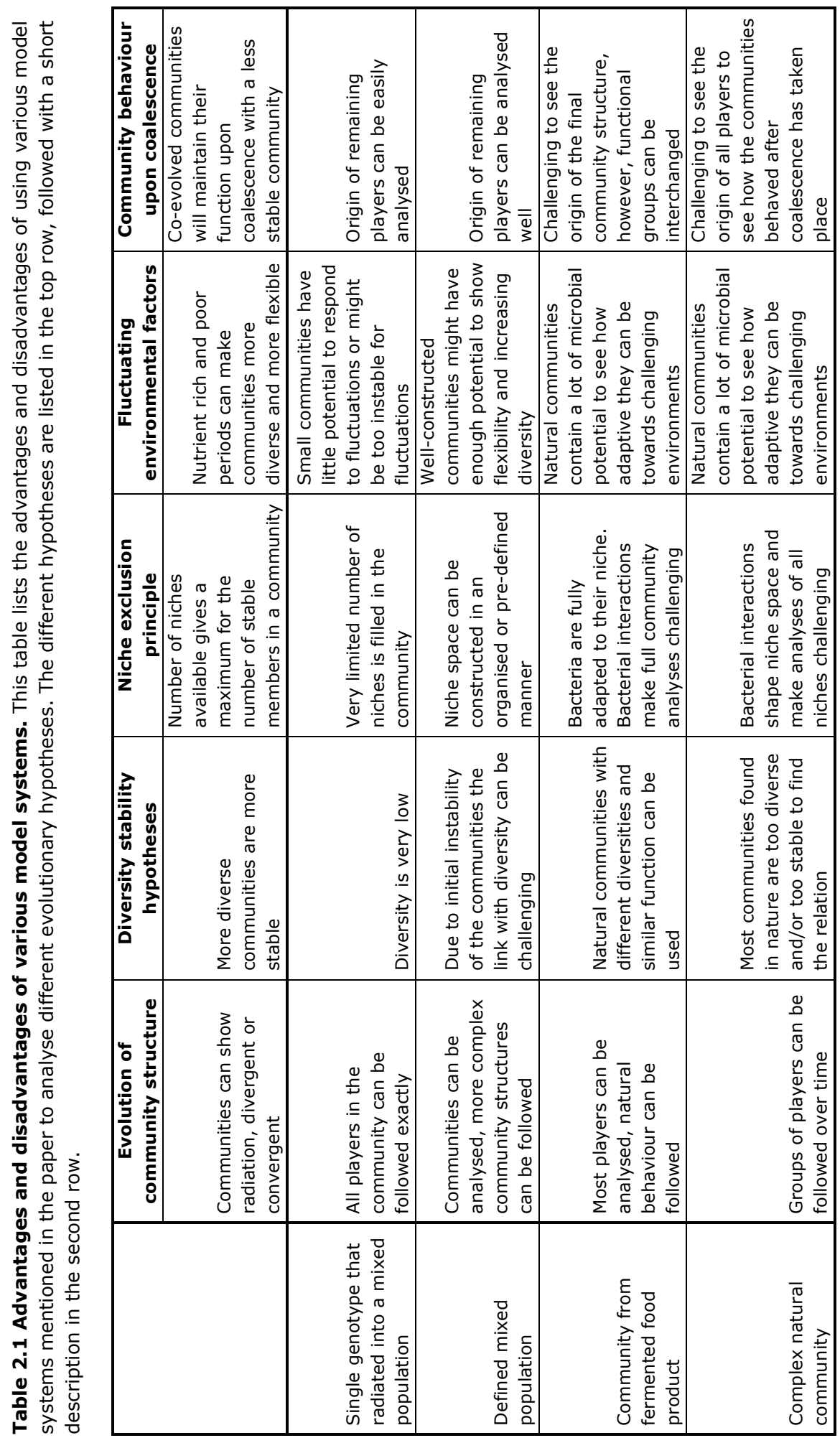


Chapter 3. 


\title{
Robust sampling and preservation of DNA for microbial community profiling in field experiments.
}

\author{
Anneloes E Groenenboom ${ }^{\mathrm{a}, \mathrm{b}}$ \\ Eddy J Smid ${ }^{b}$ \\ Sijmen E Schoustra ${ }^{a, c}$
}

Published in BMC Research Notes (2019)

\footnotetext{
a Laboratory of Genetics, Wageningen University and Research, Wageningen, The Netherlands

${ }^{b}$ Laboratory of Food Microbiology, Wageningen University and Research, Wageningen, The Netherlands

c Department of Food Science and Nutrition, School of Agricultural Sciences, University of Zambia, Lusaka, Zambia.
} 


\section{Abstract}

Objective Stabilising samples of microbial communities for DNA extraction without access to laboratory equipment can be a challenging task. In this paper we propose a method using filter paper disks for the preservation of DNA from diverse microbial communities which are found in a fermented milk product.

Results Small adaptations to the DNA extraction method used for liquid fermented milk delivered DNA of sufficient amounts and quality to be used for later analyses, e.g. full community $16 \mathrm{~S}$ amplicon sequencing. The microbial community structure obtained via the filter paper method showed sufficient resemblance to the structure obtain via the traditional DNA extraction from the liquid milk sample. This method can therefore successfully be used to analyse diverse microbial communities from fermented milk products from remote areas.

\section{Introduction}

Sequencing techniques are developing fast, resulting in more advanced and affordable ways to analyse DNA, such as characterising bacterial community structure using $16 \mathrm{~S}$ amplicon sequencing. These high tech developments however are of no use if DNA samples are not well preserved before reaching the laboratory. The stabilisation of DNA is a challenge in field experiments where researchers do not have access to a laboratory with basic equipment, like a refrigerator and a sterile work environment. In some cases the DNA extraction and amplification can be performed in the field (132). Often, biochemical solutions are being applied to stabilise the DNA (133-135). More convenient for field trials would be the use of FTA paper $(136,137)$ or another solid carrier (138), as that increases the ease of storage and sending of the samples. Such low-tech methods deliver an important advantage for field researchers who want to stabilise DNA with minimal sample processing and a few materials. In this study we explored and validated the use of simple filter paper to stabilise bacterial DNA from a fermented dairy product without prior extraction of DNA. This method is compared to an often used method where liquid samples are quickly cooled after sampling and transported in cold conditions (139).

In the method we studied, a small drop of a traditionally fermented milk product is transferred to the filter paper and left to dry. The dried paper including the drop can be stored for a long period of time at ambient temperature before the DNA extraction is performed. The subsequent DNA extraction method is a variation on the DNA extraction method used for liquid samples from fermented milk products. The dairy product used in this study, called Mabisi, is a traditionally fermented product found in rural areas in Zambia. As the fermentation in spontaneous, the bacterial community in the product is very diverse (5).

To study the composition of the microbial community in the fermented product, it is essential to quench microbial growth immediately after sampling. In a laboratory environment this would be done by cooling the sample to temperatures below $4^{\circ} \mathrm{C}$, which will slow down and stop the growth of the bacteria responsible for fermentation, but will keep them viable for later analyses. The filter paper method described here will also slow down and eventually stop bacterial growth, as it fixes the bacteria and 
dehydrates the medium. Although the bacteria will not be viable for analyses using culturing techniques, the DNA can be isolated from the papers and used for bacterial community analyses, such as $16 \mathrm{~S}$ amplicon sequencing.

\section{Methods}

\section{Preparing filter paper}

Discs of filter paper (qualitative 413,75mm, VWR International, Radnor, United States) were placed in a Petri dish. Samples of $1 \mathrm{~mL}$ were spotted at the centre of the filter paper. The filter papers were left to dry at uncontrolled temperature in a hut without windows. Drying times varied between $30 \mathrm{~min}$ and 2 hours depending on the thickness of the sample. Dried samples were stored in a Petri dish for up to two months before DNA extraction was performed.

\section{Extraction of DNA}

The DNA extraction method was adapted from Ercolini (140) and Schoustra (5). To extract bacterial DNA from the milk environment, some steps are necessary to break down the casein structure. The high protein content in combination with the relatively high percentage of lipids makes the use of the other DNA methods less successful for milk samples. For DNA extraction from liquid samples, $1 \mathrm{~mL}$ of fermented milk was spun down ( 2 minutes, 12000 RPM), after which the supernatant was removed. The cells were re-suspended in a mix of $64 \mu \mathrm{L}$ EDTA $(0.5 \mathrm{M}), 160 \mu \mathrm{L}$ Nucleic Lysis Solution (Promega, Madison, United States), $5 \mu \mathrm{L}$ RNAse $(100 \mathrm{mg} / \mathrm{ml}), 120 \mu \mathrm{L}$ lysozyme (10 $\mathrm{mg} / \mathrm{ml})$ and $40 \mu \mathrm{L}$ pronase $\mathrm{E}(20 \mathrm{mg} / \mathrm{ml})$. Samples were incubated for 60 minutes at $37^{\circ} \mathrm{C}$ while being shaken at $350 \mathrm{RPM}$.

For the extraction of DNA from the filter paper discs, a piece, 2 by 2 centimetres, was cut from the middle of the filter paper with a sterile pair of scissors. This piece was positioned at the bottom of a 1,5 mL eppendorf tube with a pair of tweezers. The cells were thoroughly suspended in a mix of $132 \mu \mathrm{l} 0.5 \mathrm{M}$ EDTA, $320 \mu$ Nuclei Lysis Solution, $1 \mu \mathrm{l}$ RNase, $240 \mu \mathrm{l}$ lysozyme and $80 \mu$ l pronase E. Samples were incubated for 24 hours at $37^{\circ} \mathrm{C}$ and agitation of 350 RPM. After the incubation step described above of both liquid samples and paper samples, a standard DNA extraction protocol was used, see supplement $A$.

\section{Bacterial community reconstruction}

For validation of the method in the laboratory the DNA of the extractions was amplified in the PCR and cloned into vectors to identify the DNA fragments in a clone library (see supplement $B$ ). The DNA extracts of the field samples, containing DNA from all organisms in the community, were sent for bacterial 16S rRNA gene amplicon pairedend sequencing of the V4 hypervariable region (341F-785R) on the MiSeq Illumina platform by LGC genomics (Berlin, Germany). For further data processing and statistics the QIIME pipeline (141), modified from Bik et al. (142) was used (see supplement C). 


\section{Results}

\section{Validation of the method in the lab}

Before testing the filter paper method in the field, the DNA extraction method was tested and optimised in the laboratory. Pure Mabisi samples and ten times diluted Mabisi samples were transferred on filter paper discs. The extracted DNA from these samples was compared to the DNA extracts from a undiluted liquid Mabisi sample. The DNA concentration in the paper with the undiluted and diluted Mabisi was $172.9 \pm 0.3$ $\mathrm{ng} / \mu \mathrm{L}$ and $11.8 \pm 1.6 \mathrm{ng} / \mu \mathrm{L}$, respectively. The amount of DNA is comparable to the DNA obtained from the liquid sample $120.7 \pm 16.5 \mathrm{ng} / \mu \mathrm{L}$. Also the purity of the DNA extracts from the filter paper is comparable to that from the liquid DNA extracts as judged by the $A 260 / A 280$ ratio $(1.80 \pm 0.05$ and $1.83 \pm 0.2$, respectively).

The clone library of the DNA extracts was blasted against the NCBI database. The microbial communities of the two liquid samples and the two paper samples with diluted Mabisi contain similar species (Supplement Figure 3.S1). Variations can be found in both abundance, richness and type of species to which these reads blast. The sample size was not sufficient to make statistical inferences.

\section{Validation of the method in the field}

At the field site in Zambia eight samples of Mabisi were split in two equal parts and subsequently transferred to filter paper as well as stored at $4^{\circ} \mathrm{C}$ before shipment to our laboratory in the Netherlands. There the DNA was extracted using the corresponding methods. The concentration of DNA obtained from these extractions varied between $25 \mathrm{ng} / \mu \mathrm{L}$ and $125 \mathrm{ng} / \mu \mathrm{L}$, with a higher average DNA concentration for the liquid samples $(84.5 \pm 25.1 \mathrm{ng} / \mu \mathrm{L})$ compared to the filter paper samples $(55.8 \pm 35.4$ $\mathrm{ng} / \mu \mathrm{L})$. To get similar concentrations of DNA for sequencing, the samples were diluted based on their DNA concentration to a concentration of 5-10 $\mathrm{ng} / \mu \mathrm{L}$. DNA extracts were sequenced (16S amplicon sequencing) using MiSeq Illumina sequencing and resulting reads were analysed to construct bacterial communities based on Operational Taxonomic Units (OTUs). Comparison of the bacterial communities present in the samples shows a high degree of similarity between samples processed via the filter paper discs and those obtained by direct extraction of the fermentation liquid (Figure 3.1). Most communities are dominated by Lactococcus lactis. In some samples also a high relative abundance of other bacterial species, belonging for example to the Acetobacter and Streptococcus genus, were found. In all cases, both collection methods (liquid and paper) show a similar microbial composition with differences in relative abundance. The differences in richness and diversity can be found in Figure 3.2, where number of OTUs, Shannon index and evenness of all samples are plotted. This analyses shows no difference in total amount of OTUs that returned, but shows a difference in OTU distribution. Communities obtained via the paper method showed a higher Shannon index and a higher evenness. 


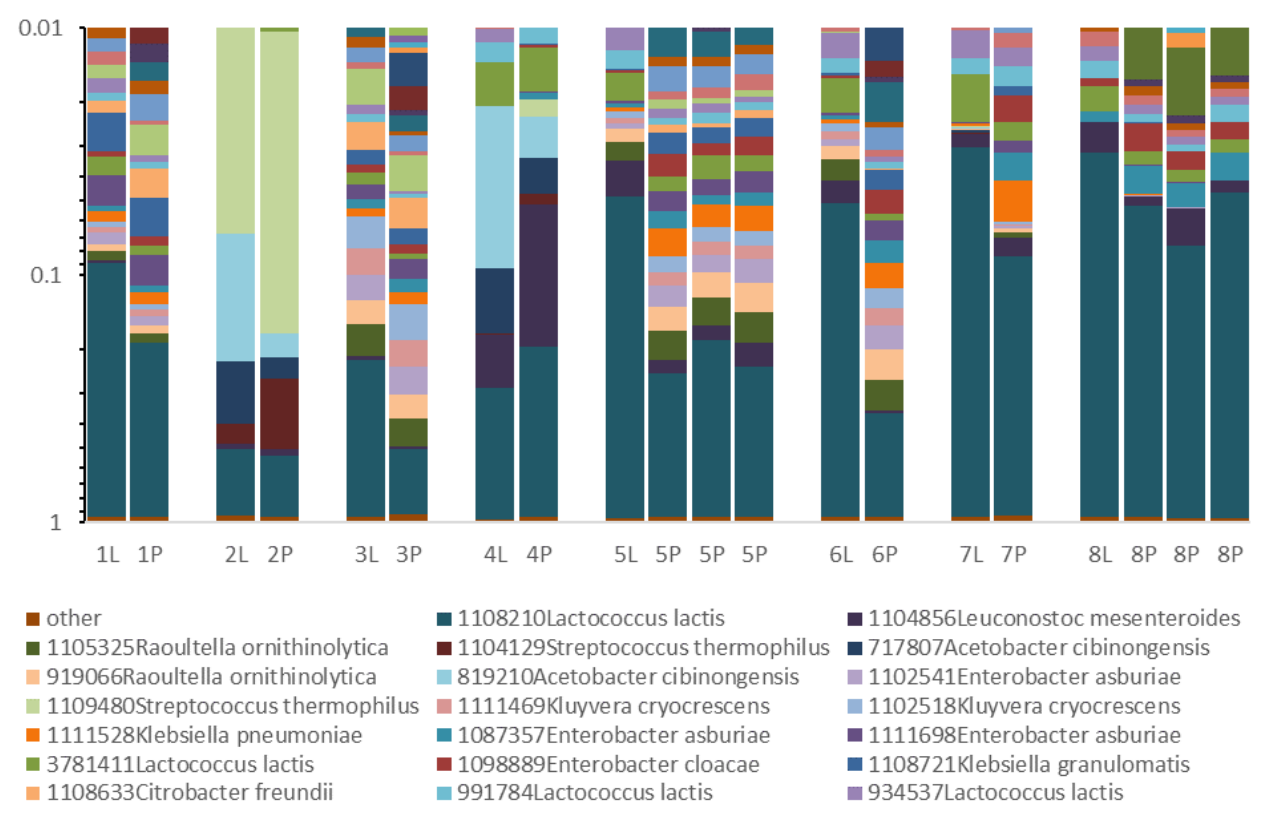

Figure 3.1 Microbial communities of paper samples and their corresponding liquid samples. Liquid samples are indicated with a L. Samples 5 and 8 have 3 technical replicates of DNA extraction from one filter paper disk. Different colours indicate different OTUs. BLAST results of the 20 most abundant OTUs are given.

\section{Discussion}

The filter paper method described above can be used to determine the bacterial microbial communities in a fermented milk products produced in areas which have no access to basic laboratory equipment and cooling facilities. With some minor adjustments to the DNA extraction method for fermented milk, good quality DNA could be extracted from the filter paper disks. To a large extent this extraction from filter paper showed the same microbial community structure as the DNA extracted from the liquid sample directly. Although the same number of OTUs were obtained from the DNA extract the distribution was slightly different. The filter paper extraction seemed to have extracted relatively less of the most abundant OTU which represent Lactococcus lactis and relatively more of the OTUs present in low amounts. Filter paper is known to have bias towards preservation of certain species (143-145), and more research towards this will be necessary to validate this bias.

To analyse all aspects of fermentation it is essential to determine the bacterial community composition during, or even before, periods of vigorous growth. With our method, in which the bacterial growth is arrested due to drying of the milk, dynamic time course samples can be stabilised and subsequently analysed at a later stage. The samples analysed here are all from fermentation end points. Consequently, no active growing microbial cells are expected anymore in these samples due to the lack of nutrients and the low medium $\mathrm{pH}$. Therefore, we can assume that at this particular stage, the bacterial community composition is already relatively stable. This makes it 
possible to compare DNA extractions from the filter paper with extraction from the liquid sample. Still the liquid sample cannot be seen as a perfect benchmark. Where the filter paper method might overestimate bacterial diversity, the DNA extracted from liquid sample might give an underestimation of the diversity. It is however a promising observation that the filter paper method was able to return low abundancy OTUs on top of identifying OTUs with high abundance. The method presented had proven its value and is sufficiently accurate for our purpose; the characterisation of the bacterial communities in a traditional fermented product. Besides, this method is easier and less expensive than the commercially available alternatives.

This method is also used for the characterisation of the bacterial communities in cereal based fermented foods from Benin, Zambia and Tanzania (unpublished observations, S. Phiri, S.E. Schoustra and A. Linnemann). Here the method also showed to be reliable and return a microbial community which is similar to a community where DNA is extracted directly from the product. The application of the presented method potentially can be extended to microbial community analysis of liquid environmental samples.
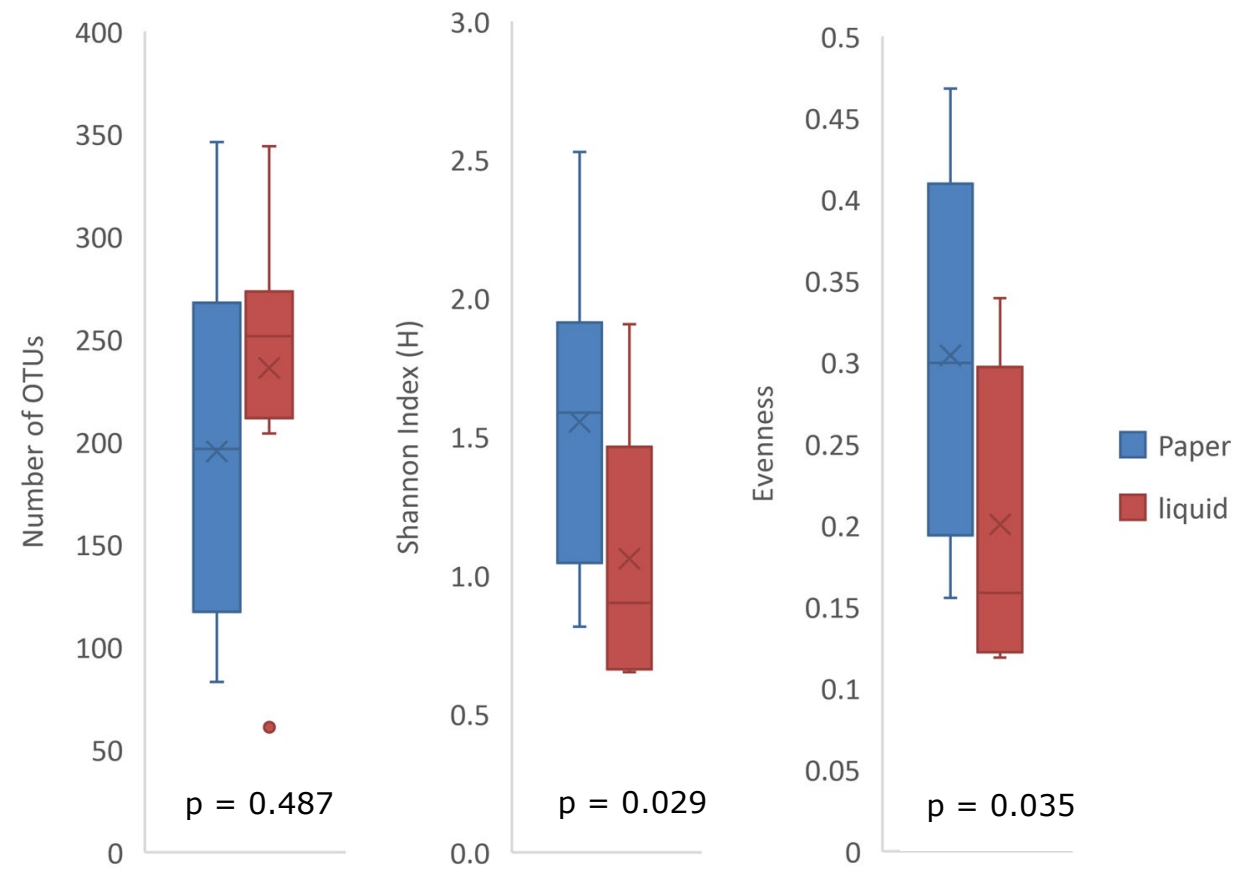

Figure 3.2 Number of OTUs, Shannon index and community evenness of filter paper samples and liquid samples. Comparison of eight samples which were all stored using the filter paper method as well as cooled to $4^{\circ} \mathrm{C}$ before shipment from Zambia to the laboratory in the Netherlands were the DNA was extracted using the corresponding methods. P values are from the related-samples Wilcoxon signed rank test and show no difference between number of OTUs, but difference in OTU distribution (in both Shannon Index and evenness). 


\section{Conclusion}

The discussed filter paper method for microbial community preservation by DNA extraction is an interesting tool to use with fermented foods in the field where other tools are not successful. Besides drying carefully in a clean environment this methods does not require any high-tech or electrical machines. This makes this method very effective for the stabilisation and transport of microbial communities in a nutritious environment like milk.

\section{Limitations}

Resulting bacterial communities show slight variation from bacterial communities constructed using DNA extracted from a liquid sample, but this is not more than variation which can be found between multiple analyses of the same DNA extracts from filter paper disks. Unfortunately, there is no golden standard for DNA stabilisation from fermented milk products to compare with this filter paper method. Another limitation is the risk for contamination. More developments in finding an optimal stabilising method for a longer storage time and a complete DNA extraction method could be done. The biggest risk arises when the papers are not dry enough. The filter paper disks do not contain any preservatives to prevent spoilage, so when the paper remains moist it could form a growth environment for both the organisms present in the sample as well as any contaminants. Regions with a dry climate, like the region in Zambia where the current study is performed, is ideal for this application, while more moist climates could cause difficulties in obtaining the right dryness.

\section{Acknowledgements}

We would like to thank the local community in Mondake, Zambia, who provided the Mabisi samples for this study. Frank Phiri and Djondoh Sikalangwe are gratefully thanked for their help during the field experiments as well as other collaborators from Heifer International Zambia. Also we thank our colleagues at the University of Zambia who helped with the preparation and lab work of this study. The help of Joost van den Heuvel in the analyses of the sequencing data is highly appreciated.

\section{Supplementary material}

\section{Supplement 3A Full DNA extraction protocol used for both filter paper and liquid samples.}

For DNA extraction from liquid samples, $1 \mathrm{~mL}$ of fermented milk was spun down (2 minutes, 12000 RPM), after which the supernatant was removed. The cells were resuspended in a mix of $64 \mu \mathrm{L}$ EDTA $(0.5 \mathrm{M}), 160 \mu \mathrm{L}$ Nucleic Lysis Solution (Promega, Madison, United States), $5 \mu \mathrm{L}$ RNAse $(100 \mathrm{mg} / \mathrm{ml}), 120 \mu \mathrm{L}$ lysozyme $(10 \mathrm{mg} / \mathrm{ml})$ and $40 \mu \mathrm{L}$ pronase $\mathrm{E}(20 \mathrm{mg} / \mathrm{ml})$. Samples were incubated for 60 minutes at $37^{\circ} \mathrm{C}$ while being shaken at 350 RPM.

For the extraction of DNA from the filter paper discs, a piece, 2 by 2 centimetres, was cut from the middle of the filter paper with a sterile pair of scissors. This piece was 
positioned at the bottom of a 1,5 mL eppendorf tube with a pair of tweezers. The cells were thoroughly suspended in a mix of $132 \mu \mathrm{l} 0.5 \mathrm{M}$ EDTA, $320 \mu$ Nuclei Lysis Solution, $1 \mu \mathrm{l}$ RNase, $240 \mu \mathrm{l}$ lysozyme and $80 \mu \mathrm{l}$ pronase E. Samples were incubated for 24 hours at $37^{\circ} \mathrm{C}$ and agitation of $350 \mathrm{RPM}$.

After the incubation step described above of both liquid samples and paper samples, a standard DNA extraction protocol was used, as follows. $400 \mu \mathrm{L}$ ice-cold ammonium acetate ( $5 \mathrm{M}$ ) was added and the mixture was cooled on ice for 15 minutes. The mixture was spun down and $750 \mu \mathrm{L}$ of supernatant was transferred to a tube containing 750 $\mu \mathrm{L}$ phenol. This tube was vortexed and its content spun down ( 2 minutes, $12000 \mathrm{RPM}$ ) and $500 \mu \mathrm{L}$ of supernatant was transferred to a tube containing $500 \mu \mathrm{L}$ chloroform. This tube was vortexed and its content spun down ( 2 minutes, 12000 RPM) and 400 $\mu \mathrm{L}$ of supernatant was transferred to a tube containing $1 \mathrm{ml} 100 \%$ ethanol and $40 \mu \mathrm{L}$ sodium acetate $(3 \mathrm{M})$. This tube containing DNA was left to precipitate at $-20^{\circ} \mathrm{C}$ overnight. The next day, the tube was spun for 20 minutes at 12000 RPM at $4{ }^{\circ} \mathrm{C}$. The supernatant was carefully aspirated and the DNA pellet was washed by adding $1 \mathrm{~mL}$ $70 \%$ ethanol. The tube was spun for 10 minutes at 12000 RPM at $4{ }^{\circ} \mathrm{C}$, after which the supernatant was aspirated again. The DNA pellet was left to dry at room temperature and dissolved in $20 \mu \mathrm{L} 10 \mathrm{mM}$ Tris $\mathrm{pH}$ 7.5. DNA concentration were measured using Nanodrop 2000/2000c UV-VIS (ThermoFisher, Waltham, US).

\section{Supplement 3B Analysis of community structure using clone libraries.}

For the validation in the laboratory prior to the larger scale field validation experiments, community profiles were analysed using clone libraries. DNA extracts were amplified in the $16 \mathrm{~S}$ region with a PCR with $16 \mathrm{~S}$ primers 27F-1492R (Biolegio, Nijmegen, Netherlands) and the following amplification conditions: $94^{\circ} \mathrm{C}$ initial denaturation, 10 min; then 34 cycles of $94{ }^{\circ} \mathrm{C}, 30 \mathrm{sec} ; 55^{\circ} \mathrm{C}, 30 \mathrm{sec} ; 72{ }^{\circ} \mathrm{C}, 1 \mathrm{~min}$, followed by a 72 ${ }^{\circ} \mathrm{C}$ final extension, $10 \mathrm{~min}$. PCR products were cleaned using a PCR clean-up kit (Macherey-Nagel, Düren, Germany) according to the manufacturer's instructions. Clone libraries were constructed using the StrataClone PCR Cloning Kit (Stratagene, San Diego, California, United States) according for the manufacturer's instructions. Resulting clone libraries were sequenced using Sanger sequencing (Eurofins Scientific, Brussels, Belgium) with T3 primer. Sequence data were compared to the NCBI database using BLAST (146). Matches from the blast search were used to construct the bacterial community. Results are in Figure 3.S1.

\section{Supplement 3C Analysis of 16S rRNA amplicon sequencing data for bacterial community reconstruction.}

The extract containing DNA from all organisms in the community was sent for bacterial $16 \mathrm{~S}$ rRNA gene amplicon paired-end sequencing of the V4 hypervariable region (341F$785 \mathrm{R}$ ) on the MiSeq Illumina platform by LGC genomics (Berlin, Germany). For further data processing and statistics the QIIME pipeline (141), modified from Bik et al (142) was used as follows. Paired-end reads were joined using join_paired_ends.py (with minimum overlap 10 basepairs) after which sequences were trimmed and filtered using cutadapt (v1.11 -q 20, -m 400, Martin 2011) using the known primer sequences CCTACGGGNGGCWGCAG and GACTACHVGGGTATCTAAKCC to trimmed both sides of 
the sequence. These trimmed sequences were then checked for chimera's, using uchime (v4.2.20, gold database, Edgar et al 2014), and sequences with a chimera score lower than 0.28 were retained. After the trimming and filtering steps sequences were clustered (UPGMA) into operational taxonomic units (OTUs) after quality check using pick_open_reference_otus.py (-s 0.1 , -enable_rev_strand_match TRUE, align_seqs_min_length 75, -pick_OTU_similatiry 0.95). Taxonomy of the resulting OTUs was assigned to representative sequences using the Greengenes (v13.5) rRNA database. This algorithm gives a representative sequence for an OTU, which were used to perform a local blast using the gold database from uchime. The taxonomy from the top BLAST hit was used for further data processing.

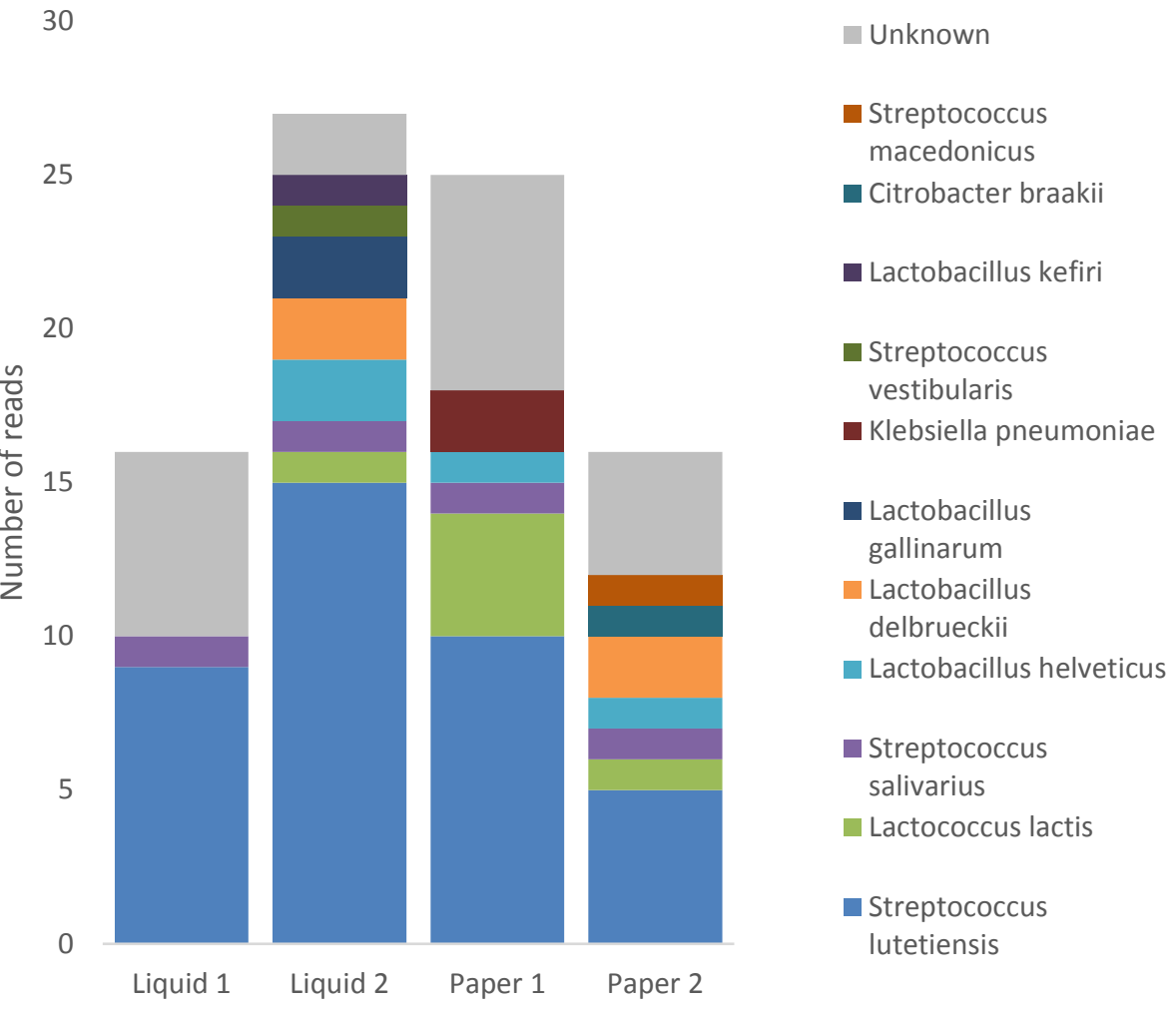

Figure 3.S1. Bacterial composition of liquid and paper samples all originating from the same Mabisi sample. Different colours indicate different species which could originate from different reads. Numbers 1 and 2 indicate the two technical replicates of DNA extraction. 
Chapter 4. 


\title{
Microbial population dynamics during traditional production of Mabisi, a spontaneously fermented milk product from Zambia. A field trial.
}

\author{
Anneloes E Groenenboom ${ }^{a, b}$ \\ John Shindanoc \\ Nachimuka Cheepad \\ Eddy J Smid \\ Sijmen E Schoustra ${ }^{a, c}$
}

\footnotetext{
a Laboratory of Genetics, Wageningen University and Research, Wageningen, The Netherlands

b Food Microbiology, Wageningen University and Research, Wageningen, The Netherlands

c Department of Food Science and Nutrition, University of Zambia, Lusaka, Zambia

d Heifer international Zambia, Lusaka, Zambia
} 


\section{Abstract}

Mabisi is a fermented milk traditionally produced in a calabash by uncontrolled fermentation. Due to high costs and a reduced availability of calabashes, nowadays more and more plastic containers are used for Mabisi production. However, the effect of this change in production practice on the properties of the product is unknown. Therefore, here we aim to determine the effect of fermentation vessels and types of back-slopping on acidification and microbial communities during fermentation. A series of fifteen experiments using two types of fermentation vessels (plastic buckets and calabashes) in combination with different types of back-slopping (no back-slopping, passive back-slopping, and active back-slopping) were set up at a field site in rural Zambia. In each of the fifteen fermentations we analysed acidification rate of traditional Mabisi fermentation and bacterial diversity over time. No significant difference was found in terms of microbial diversity during and at the end of fermentation between fermentations performed in buckets or previously used calabashes. Bacterial communities in general decreased in diversity over time, where the drop in $\mathrm{pH}$ correlated with a decrease in Shannon Index. In case of active back-slopping, the $\mathrm{pH}$ drop started right after inoculation while in the no back-slopping and passive backslopping fermentations, there was a clear lag phase before acidification started. All experimental series resulted in a microbial community dominated by Lactococcus lactis and a Shannon Index as measure for diversity between 0.6 and 2.0. The use of plastic buckets for Mabisi fermentation can be a valuable alternative to the use of calabashes as this study showed no biological and physico-chemical differences between Mabisi resulting from both fermentation vessels, although the reason for perceived differences should be further investigated.

\section{Introduction}

Traditional products made by spontaneous fermentation are an important aspect of culture, nutrition and livelihoods in many countries. Mabisi is such a product found in many rural areas in Zambia. Mabisi is fermented cow milk traditionally produced in a calabash by uncontrolled fermentation that is dominated by six to ten different species of lactic acid bacteria (5). Households have their own calabashes that they use for consecutive rounds of Mabisi production. The methods of producing Mabisi vary depending on location and can include back-slopping, churning, and other production methods (8). The 'Tonga-type' is the most simple and commonly practiced way of producing Mabisi, in which milk is sieved before allowing it to incubate statically at ambient temperature for 24 to 48 hours in a calabash and is stirred right before consumption (8). The moment of consumption is determined by organoleptic observations: when the Mabisi is thick and sour enough it is ready for consumption.

Due to high costs and a reduced availability of calabashes, nowadays more and more plastic and metal containers are used for Mabisi production. The main process remains unchanged: fresh raw milk is collected in the container and left to ferment for 24 to 48 hours at ambient temperatures. Although this method is accepted nationwide, there is the general belief that milk fermentation in calabashes results in a Mabisi with better organoleptic characteristics than Mabisi produced in plastic containers. This perceived difference might be caused by differences in the bacterial communities present in the fermenting milk. These fermenting bacteria will form most of the volatile aroma 
compounds and are responsible for other specific product attributes such as viscosity, which together determine the flavour and overall organoleptic properties of the Mabisi. A more diverse microbial community might result in a product with a richer flavour palate as it is likely to produce a higher variety of aromas (147-150) and it increases the chance of high flavour forming strains being present (151).

In spontaneous fermentation such as in the production of Mabisi, bacteria can enter the raw milk and start the fermentation from various sources. As the milk is not pasteurised, all bacteria that were already present or entered the milk via the air or the milking equipment used, will still be present at the onset of fermentation. This can be the case in both types of fermentation vessels, calabashes and buckets. A key difference, however, is the inner surface of both vessel types. Where buckets have a smoother surface, which can be washed and dried more easily between fermentation cycles, the calabashes are made of natural material and have a rough inner surface. The calabash surface provides a great opportunity for bacteria to form biofilms and hide in small cracks without being removed during washing and drying. Most likely, the bacteria that remain in the calabashes after a previous fermentation cycle are essential for starting a new fermentation cycle (152). Without this rough surface, fermentation using buckets might result in a less diverse microbial community and therefore in a less rich product. Back-slopping might increase the diversity in the buckets to a level comparable to calabashes. Here, we define two types of back-slopping: 1) active backslopping, where finished product is added to the raw milk to start the fermentation, and, 2) passive back-slopping, meaning the un-intentional transfer of bacteria between two rounds of fermentation by using the same fermentation vessel.

There are no studies on the influence of fermentation vessels and types of backslopping on the spontaneous fermentation of Mabisi in Zambia. Therefore, a field study was conducted in rural Zambia to determine the influence of two specific variables on the rate of acidification and on the microbial community present in the final product of Mabisi: 1) the type of fermentation container used, a plastic bucket or a calabash, and, 2 ) the type of back-slopping between different rounds of fermentations. Since we used traditional methods of spontaneous fermentation, several variables were uncontrolled, such as temperature and batch volumes. We nevertheless monitored these uncontrolled variables, to gain insight in the potential effects of these variables on the overall fermentation trajectory and specific characteristics of the fermentation process.

\section{Materials and methods}

\section{Field site}

Experiments were performed at the Mondake Dairy Association based in the rural part

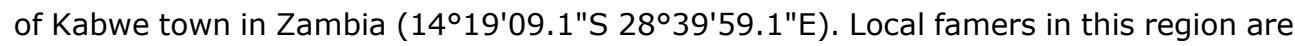
supported by Heifer Zambia, an NGO that provides dairy cows and training in animal husbandry to fight hunger in rural areas. As the morning milk was collected to be sold to a nationwide operating dairy company, only the afternoon milk was used for this study (Figure 4.1A). To prevent food waste, all afternoon milk that was made available was used for the fermentations, which resulted in variations in fermentation volumes (Table 4.1). As local customs and traditions were taken into account, also the starting time of fermentation and the sampling regime varied per fermentation. The 
experiments were conducted in July, and more information about the local community and the field experiment can be found in Supplement Figure 4.S1 and Supplement 4A.
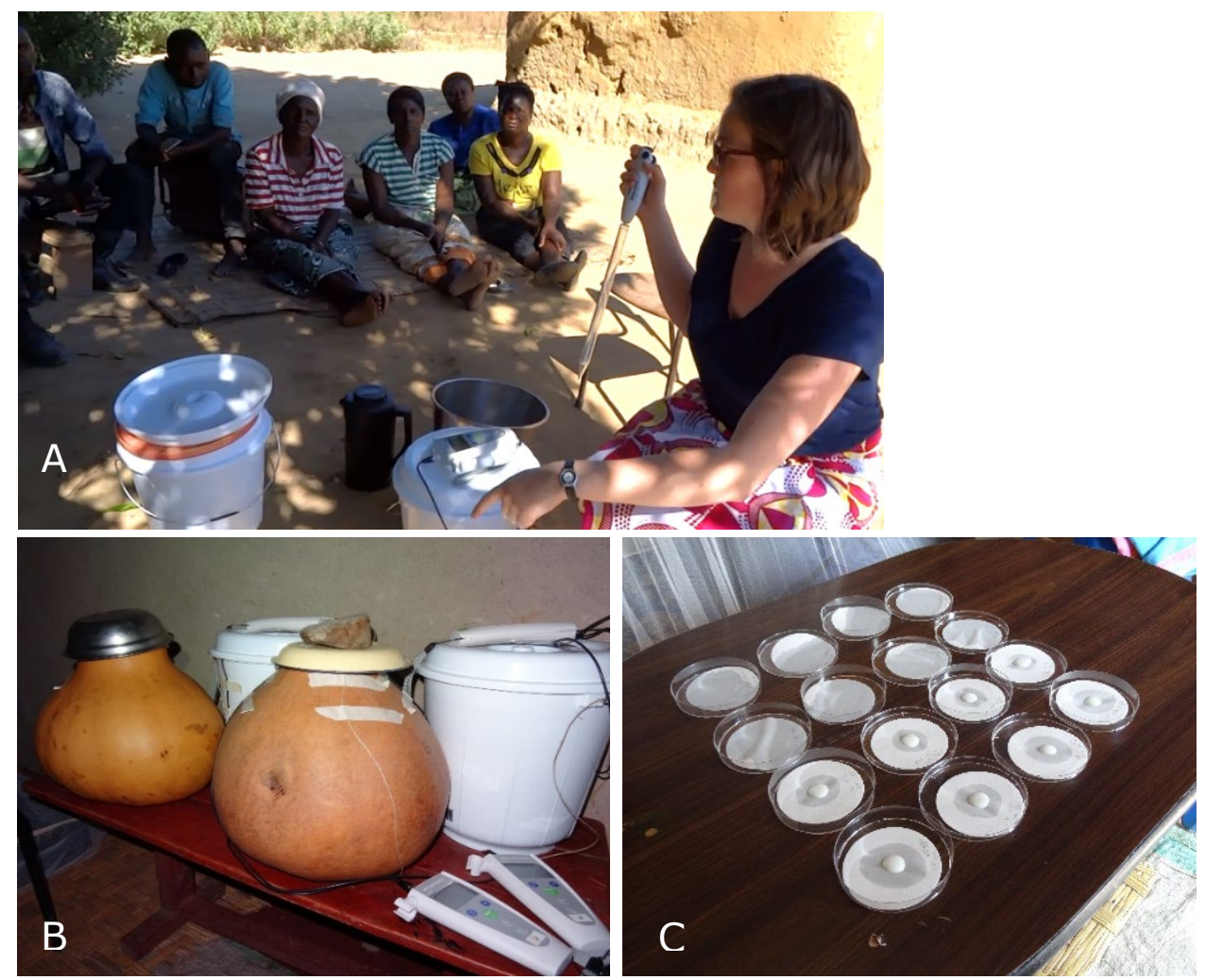

Figure 4.1 Set-up of the field-experiments. Panel (A) In the afternoon the fermentations were started. Active backslopping was performed using a pipette. The local community showed great interest in the study. (B) Fermentation was performed in calabashes and plastic buckets, with $\mathrm{pH}$ meters and temperature probes attached. At night the containers were put inside the house to prevent temperature drops. (C) Samples of Mabisi at different stages of fermentation were stabilised on a filter paper.

\section{Experimental setup in the field}

In total, we performed fifteen fermentation cycles, producing Mabisi from raw milk (Table 4.1). Three ways of back-slopping were applied (not, passive, and active) in two types of fermentation vessels (calabashes and plastic buckets). Passive backslopping in buckets was considered to be non-existing, as the buckets were thoroughly cleaned with water after every fermentation round. This resulted in five different treatments for fermentation (Table 4.2). The availability of the equipment (fermentation vessels and $\mathrm{pH}$ and temperature probes) and the amount of milk, caused the experiments to be conducted over the course of 14 days. Five fermentations could be monitored simultaneously in terms of temperature and $\mathrm{pH}$. 
Three calabashes (about 20 L capacity) were obtained from local markets, two of the calabashes have been provided to the Mabisi producers two months before the start of the study to be used for Mabisi production. One calabash has never been used for Mabisi production before the study which made it possible to perform one non backslopped fermentation in that vessel type. The calabashes used for all other fermentations had been used before and were therefore considered to be a source of inoculum for the next fermentation cycle via passive back-slopping. According to traditional practice, calabashes were all filled with water and a mix of grass and cane and left for a day to prepare to be used for Mabisi fermentations. Between fermentation cycles, the calabashes were washed with water. All water used was untreated water from a small stream, according to common practice and availability. Three white buckets with lids ( 20 L capacity) were bought from a nationwide grocery store and brought to the field site. Buckets were cleaned with water before and after fermentation rounds.

Fermentation volumes were dependent on the availability of milk. The milk production of the cows was variable, as well as the availability of the farmers that would milk the cows and deliver the milk to the field location. Milk was obtained from up to six farmers, but mostly three farmers. The milk was combined into one batch and sieved right before it was put in the fermentation vessels.

Fermentation vessels were equipped with temperature probes (Thermochron High resolution $15 / 46^{\circ} \mathrm{C}$, Sydney, Australia) in water tight capsules (Thermochron, Sydney, Australia) and pH-probes (FiveGo, Mettler Toledo, Schwerzenbach, Switzerland), continuously placed in the fermenting medium (Figure 4.1B). The starting time of fermentation was dependent on the milking time of the farmers and was typically between 1PM and 4PM. For active back-slopping, the absolute amount of finished Mabisi was dependent on the volume of the new fermentation; the fresh milk was inoculated with $1 \%(\mathrm{v} / \mathrm{v})$ finished Mabisi at the start of a new fermentation cycle. For passive back-slopping and no back-slopping no further actions were taken. During the day the fermentation vessels were positioned outside in the sun or in the shade. During the night the containers were stored in a hut which was heated by hot coals. Table 4.3 shows all (un)controlled and (un)measured variables.

\section{Sampling of Mabisi for Microbial Analyses}

For the first eleven fermentations (Table 4.1) samples of the fermentation liquid were taken during fermentation using sterile pipets (Greiner Bio-One, Frickenhausen, Germany) and a Smoothie pipet filler (VWR/Avantor, Radnor, USA). Sampling times were typically at $0 \mathrm{~h}, 0.5 \mathrm{~h}, 2 \mathrm{~h}, 6 \mathrm{~h}, 16 \mathrm{~h}, 20 \mathrm{~h}, 24 \mathrm{~h}, 40 \mathrm{~h}$ and $48 \mathrm{~h}$ after the start of fermentation, but varied per fermentation cycle due to circumstances of the field site (such as cultural obligations and night time). These samples were stabilised on filter papers as described in Groenenboom et al. (153) to allow analysis of microbial community composition (Figure 4.1C). Liquid samples at the end of fermentation were also stored on ice. Samples on ice were picked up once per day and transported to the Heifer International (Zambia) local office $50 \mathrm{~km}$ away, to be stored in a refrigerator at $7^{\circ} \mathrm{C}$ for two to four weeks. All samples were subsequently air shipped to the Laboratory of Genetics at Wageningen University, the Netherlands for DNA extraction $(140,153)$. The extracted DNA is the starting material for the bacterial community analyses. 
Table 4.1. Details of all fifteen fermentations. Time indicates moment at which milk was poured into the fermentation vessels. This field experiment was performed in 2015.

\begin{tabular}{|c|c|c|c|c|c|c|c|}
\hline & DATE & TIME & VESSEL & BACK-SLOPPING & \multicolumn{2}{|c|}{$\begin{array}{l}\text { VOLUME (L) } \\
\text { TOTAL/IN VESSEL }\end{array}$} & $\begin{array}{l}\text { T \& PH } \\
\text { MEASURED }\end{array}$ \\
\hline 1 & 16 July & $13: 40$ & Calabash & Passive & 2.5 & 2.5 & \multirow{11}{*}{ yes } \\
\hline 2 & \multirow{2}{*}{17 July } & \multirow{2}{*}{$14: 40$} & Bucket & No & \multirow{2}{*}{6.5} & 3.25 & \\
\hline 3 & & & Bucket & Active & & 3.25 & \\
\hline 4 & 18 July & $15: 00$ & Calabash & Passive & 3.5 & 3.5 & \\
\hline 5 & 19 July & $16: 15$ & Calabash & Active & 9.5 & 9.5 & \\
\hline 6 & 20 July & $15: 30$ & Calabash & No & 11 & 11 & \\
\hline 7 & \multirow{3}{*}{21 July } & \multirow{3}{*}{$15: 15$} & Calabash & Active & \multirow{3}{*}{23} & 7.77 & \\
\hline 8 & & & Bucket & Active & & 7.67 & \\
\hline 9 & & & Bucket & No & & 7.66 & \\
\hline 10 & 22 July & $16: 30$ & Calabash & Passive & 8 & 8 & \\
\hline 11 & 23 July & $15: 30$ & Bucket & No & 7 & 7 & \\
\hline 12 & \multirow{4}{*}{27 July } & \multirow{4}{*}{$13: 00$} & Calabash & Active & \multirow{4}{*}{18.5} & 4.63 & \multirow{4}{*}{ No } \\
\hline 13 & & & Bucket & Active & & 4.62 & \\
\hline 14 & & & Bucket & No & & 4.63 & \\
\hline 15 & & & Calabash & Passive & & 4.62 & \\
\hline
\end{tabular}

Table 4.2 The summary of conditions of all fifteen fermentations. Fermentation are performed in calabashes or buckets as fermentation vessel and back-slopped or not. An unequal number of fermentations per treatment was caused by: 1 ) One new calabash was available to perform a not back-slopped fermentation. 2) Passive back-slopping is not considered possible in plastic buckets due to the smooths surface on the inside of the bucket. 3) Due to time constrains two fermentations with active back-slopping could not be performed.

\begin{tabular}{l|l|l|l} 
& Not back-slopped & $\begin{array}{l}\text { Passively } \\
\text { back-slopped }\end{array}$ & $\begin{array}{l}\text { Actively } \\
\text { back-slopped }\end{array}$ \\
\hline Calabash & 1 & 4 & 3 \\
\hline Bucket & 4 & - & 3
\end{tabular}

\section{Analyses of Bacterial communities}

The extracts containing DNA from all organisms in the microbial community were sent for bacterial 16S rRNA gene amplicon paired-end sequencing of the V4 hypervariable region (341F-785R) on the MiSeq Illumina platform by LGC genomics (Berlin, Germany).

For further data processing and statistics the QIIME pipeline (141), modified from Bik et al. (142) was used. Paired-end reads were joined using join_paired_ends.py (with minimum overlap 10 base pairs) after which sequences were trimmed and filtered using cut adapt (v1.11 -q 20, -m 400 (154)) and using the known primer sequences 
CCTACGGGNGGCWGCAG and GACTACHVGGGTATCTAAKCC to trim both sides of the sequence. These trimmed sequences were then checked for chimera's, using UCHIME (v4.2.20, gold database (155)); sequences with a chimera score lower than 0.28 were retained. Next, sequences were clustered into operational taxonomic units (OTUs) after quality check using pick_open_reference_otus.py (-s 0.1 , -enable_rev_strand_match TRUE, -align_seqs_min_length 75, -pick_OTU_similatiry 0.95). Taxonomy of the resulting OTUs was assigned to representative sequences using the Green genes (v13.5) rRNA database. This algorithm gives a representative sequence for an OTU, which were used to perform a local BLAST using the gold database from uchime (146). Shannon index $(\mathrm{H})$ accounts for both number and evenness of OTUs present and is calculated using:

$$
H=-\sum_{i=1}^{s}\left(p_{i} \ln p_{i}\right)
$$

in which $\mathrm{p}_{\mathrm{i}}$ is the proportion of reads belonging to category $\mathrm{i}$, and $\mathrm{s}$ is the total number of categories which can be OTUs, species, or genera depending on the level of clustering of the reads. The Shannon index can lie between 0 and In s (156), depending on the distribution of the categories (evenness). The number of identified OTUs (s) in Mabisi communities ranges from about 40 (typically at the beginning of fermentation) to 400 (typically at the end of fermentation) per sample, giving a maximal Shannon index between 3.7 and 6.0 (In s). Pairwise Spearman's correlation of Shannon index values and $\mathrm{pH}$ was estimated for samples obtained using filter paper method.

Table 4.3 Variables which were (un)measured and (un)controlled during the field fermentations.

\begin{tabular}{l|l|l} 
& Unmeasured variables & Measured variables \\
\hline $\begin{array}{l}\text { Uncontrolled } \\
\text { variables }\end{array}$ & $\begin{array}{l}\text {-Time between milking and } \\
\text { 'start' of fermentation } \\
\text {-Cows from which the milk } \\
\text { originated }\end{array}$ & $\begin{array}{l}\text {-Temperature during } \\
\text { fermentation cycle } \\
\text {-Fermentation volume } \\
\text {-Start time of fermentation }\end{array}$ \\
\hline $\begin{array}{l}\text { Controlled } \\
\text { variables }\end{array}$ & - & $\begin{array}{l}\text {-Fermentation vessel type } \\
\text {-Backslopping method }\end{array}$ \\
\hline Output variables & - & $\begin{array}{l}\text {-pH } \\
\text {-Microbial community } \\
\text { composition during } \\
\text { fermentation }\end{array}$
\end{tabular}




\section{Results}

\section{Documentation of uncontrolled variables}

Ambient temperatures in July in Zambia are quite predictable. Normal outside day temperatures are in the range of 22 to $25^{\circ} \mathrm{C}$ (Figure 4.2B). The fermentations were performed outside during the day and inside during the night, allowing temperatures to increase under the influence of sunlight during the day and tempering the cooling down during the night when outside temperatures drop to as low as $5{ }^{\circ} \mathrm{C}$. The temperatures inside the fermentation vessels are not only influenced by the outside temperature but also by vessel type and volume of the fermented milk. The fermentation vessels used have a different shape and are made from different material, resulting in differences in isolation and the ability for evaporation. On average the temperature of the milk in buckets increased at a faster rate and reached a higher level than the milk in calabashes (Figure 4.2). During the night the milk in both fermentation vessel types cooled down to a similar level. After around 24 hours of fermentation the difference in milk temperature between the two types of fermentation vessels was the largest. Apart from the vessel type, the volume of the milk can affect the temperature profile. The average volume in calabashes was a bit higher than the average volume in the buckets: $7.0 \pm 3.1 \mathrm{~L}$ and $5.8 \pm 2.1 \mathrm{~L}$, respectively. When we plot the maximum temperature difference per fermentation as a function of volume, we see a nonsignificant negative correlation (Figure 4.3).
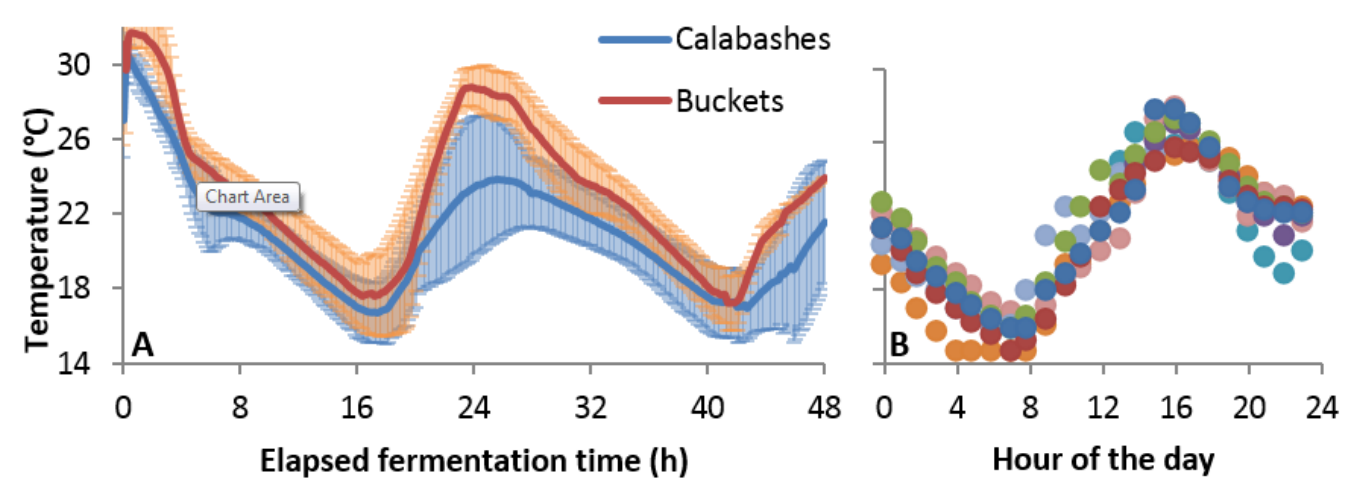

Figure 4.2 Temperature during fermentation A) Temperatures measured using a temperature button in the milk during fermentation. Lines show averages of all fermentations using a calabash (blue line) and a bucket (red line). Error bars indicate standard error from the average calculated over 6 fermentations in calabashes and 5 fermentations in buckets. Start of fermentation ( $t=0 \mathrm{~h}$ ) happened at different time of the day for all fermentation but was typically between 1PM and 4PM. B) Daily air temperatures measured inside the hut where the fermentation vessels were stored at night for the period between 16th and 27th July 2015. Different colours indicate different days. The temperature buttons could not measure below $14^{\circ} \mathrm{C}$. 


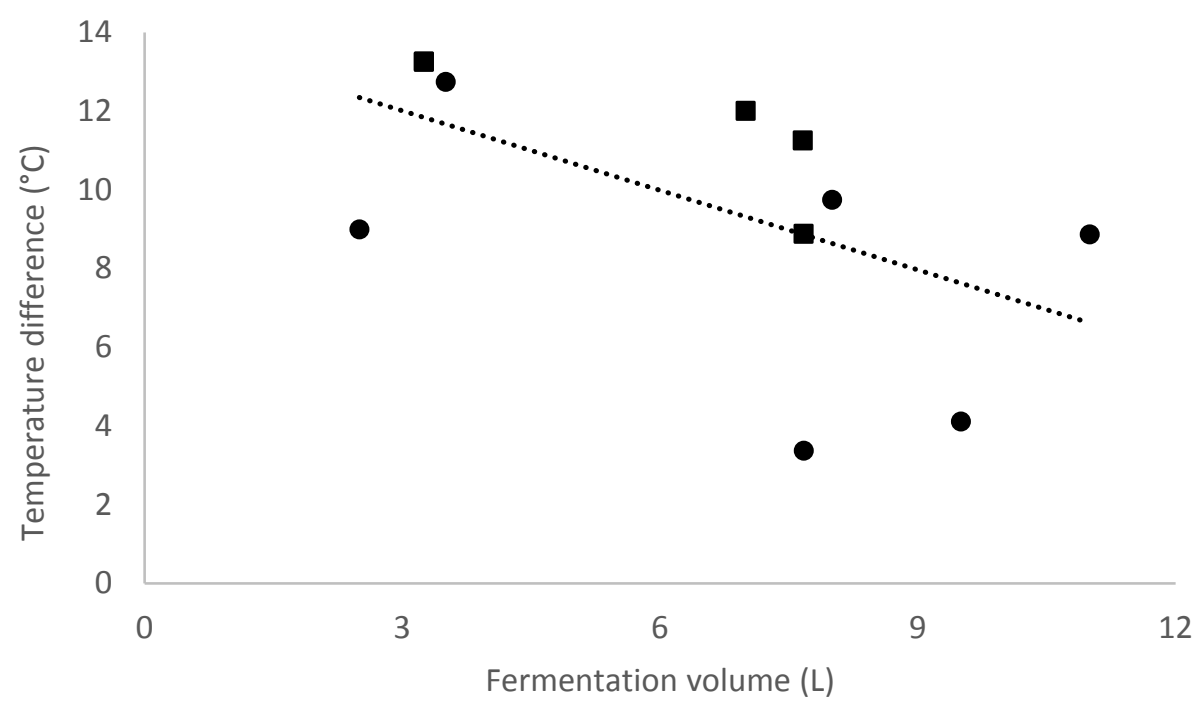

Figure 4.3 Temperature change as a function of volume of milk used for fermentation in calabashes $(\bullet$ ) and buckets ( $\mathbf{})$. Temperature change is the difference in temperature between the minimal (around 16 hours after the start of fermentation) and maximal (around 24 hours after the start of fermentation) measurement during the fermentation cycle. Pearson correlation: $r=-0.568$, $p=0.068$.

\section{Acidification}

The $\mathrm{pH}$ was measured during all fermentations in the five different treatments (Table 4.1). Results are given in Figure 4.4 and showed that in all cases the $\mathrm{pH}$ had dropped below $\mathrm{pH} 5$ after $48 \mathrm{~h}$. The time elapsed before the $\mathrm{pH}$ dropped below $\mathrm{pH} 5$ varied. When a new calabash or a new or not back-slopped bucket was used, the drop in $\mathrm{pH}$ as measure for fermentation activity had a lag time of about 18 hours. This lag time was not present in the fermentations that were actively back-slopped; the $\mathrm{pH}$ decreased below 5 within 18 hours. In case of passive back-slopping due to the re-use of a cleaned calabash the results were mixed; in one case the lag time was even longer than 18 hours while in other fermentations the $\mathrm{pH}$ was below 5 within 24 hours. 


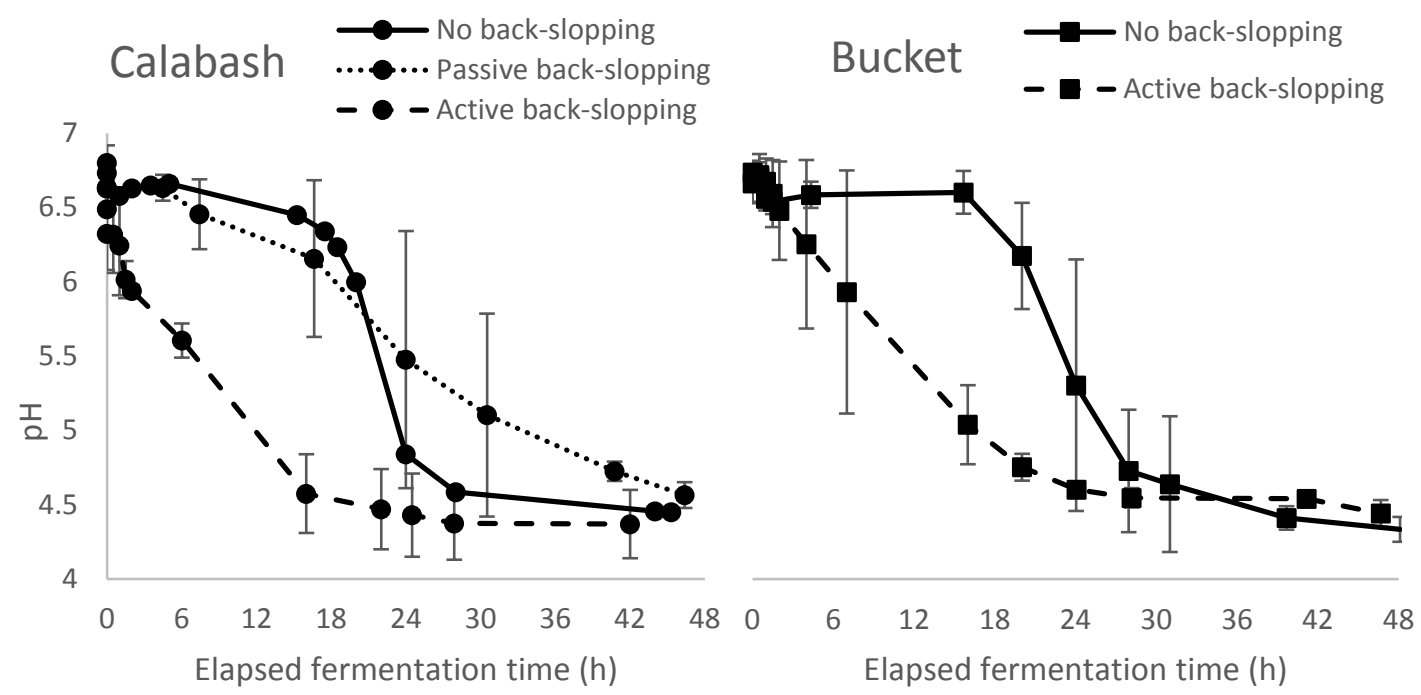

Figure 4.4 pH over time during fermentation with no back-slopping and passive and active back-slopping in calabashes and no back-slopping and active back-slopping in buckets.

\section{Microbial community composition}

Samples were shipped to the laboratory in the Netherlands where DNA was extracted. The extracts were sent for $16 \mathrm{~S}$ amplicon sequencing and the resulting reads were analysed to construct bacterial community compositions (Figure 4.5). On average the most abundant species were found to be from the genera Lactococcus, Leuconostoc, Streptococcus, Acinetobacter and Acetobacter. The most abundant OTU (between 43\% and $96 \%$ of all OTUs) returned as a Lactococcus lactis strain from the BLAST analysis. This OTU was most abundant in all samples towards the end of fermentation (after $40 \mathrm{~h}$ or more) for both buckets and calabashes. In samples taken at the beginning of fermentation (within the first $5 \mathrm{~h}$ ) more diverse bacterial communities are found containing various genera that were not found at later time points of fermentation, such as Mycoplasma and Clostridium species.

The Shannon index of the bacterial communities was calculated as a measure for community diversity (Supplement Figure 4.S2). New calabashes stand out in that they harbour microbial communities of higher diversity than observed in the other treatments of used calabashes or buckets. Furthermore, there is no difference in diversity when different ways of back-slopping were used. The end-point samples obtained from all fifteen fermentations provide an overview of the results of all five methods (Figure 4.6). No difference in final community structure was found between different ways of back-slopping or the use of fermentation vessel. Considering active back-slopping we observed a trend towards a higher microbial diversity in the buckets compared to the diversity found in calabashes. In buckets, active back-slopping appeared to increase the communities' bacterial diversity compared to bacterial communities of fermentations without back-slopping. 


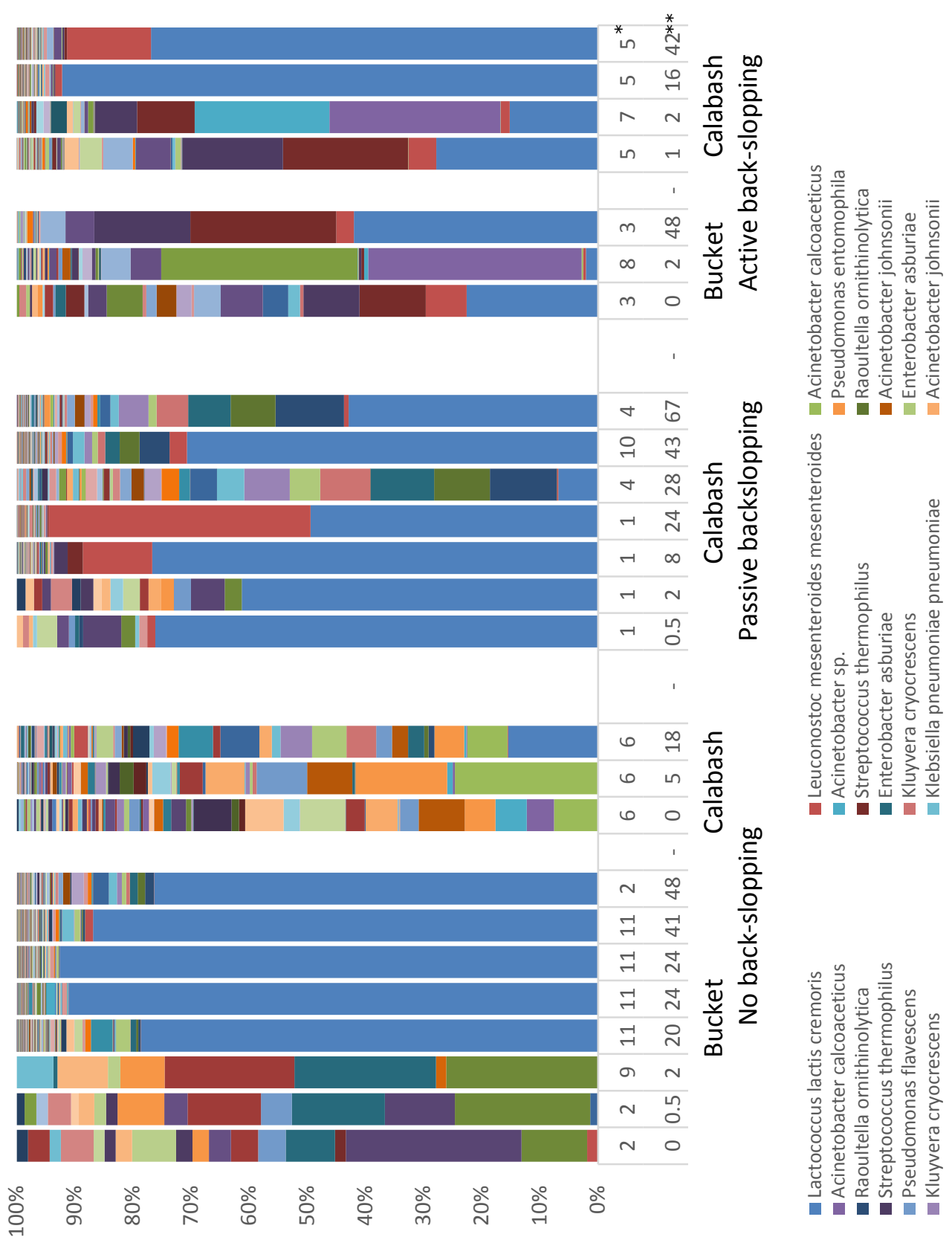

Figure 4.5 Bacterial community structure of Mabisi samples during the course of fermentation obtained with the paper method. Different colours represent different OTUs. Total number of OTU per sample taken as $100 \%$. BLAST results of the most abundant 21 OTUs are given. *Fermentation round, details in Table 4.3. **Time after start of fermentation in hours. Some samples could not be included because the field experiment did not allow regular sampling and some samples were lost due to contamination. 


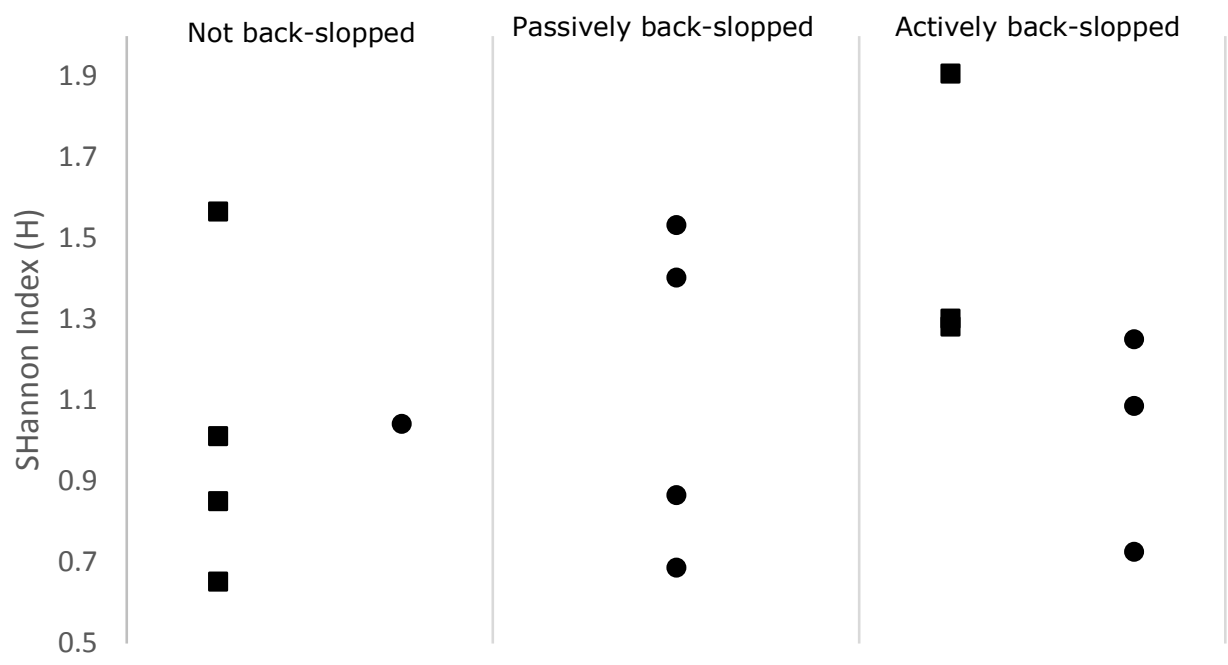

Figure 4.6 Shannon Index of microbial communities at the end of fermentation in calabashes $(\bullet)$ and buckets $(\mathbf{\bullet})$. Only one fermentation was performed in a new calabash and due to the smooth inner surface, passive back-slopping in buckets was neglected. No significant differences between fermentation could be found, possibly due to lack of statistical power.

Interestingly, at a lower $\mathrm{pH}$ the overall diversity of a community was lower (Figure 4.7). This correlation was uncovered using all available samples obtained via the filter paper method during fermentation. Although the acidification rate in the different fermentation methods had different rates, this clearly correlates with the community diversity.

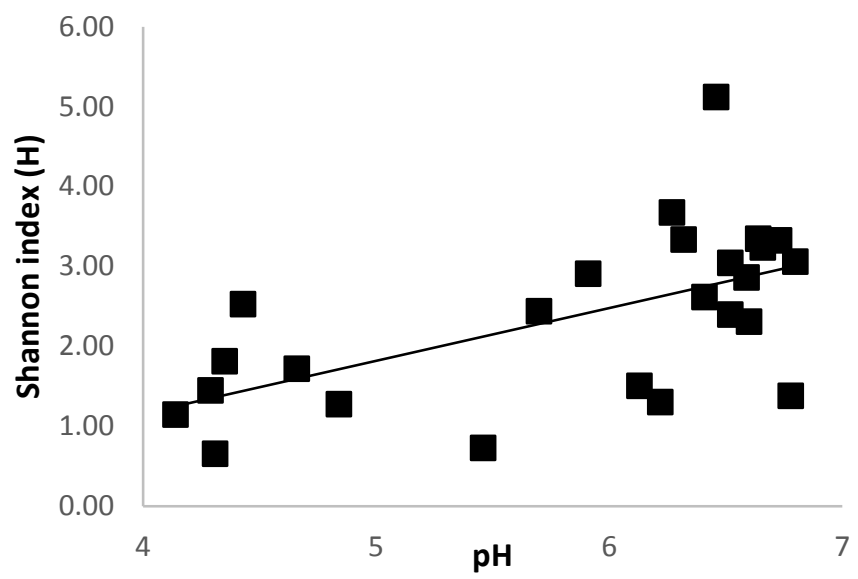

Figure 4.7. Shannon index (based on OTU) plotted as a function of pH indicated a significant positive correlation between the two. The $\mathrm{pH}$ lowered as the fermentation progressed which led to a lower species diversity (Spearman's correlation coefficient $=0.582, d f=23, p=0.002$ ). 


\section{Discussion}

This study focussed on the spontaneous fermentation of Mabisi using traditional calabashes and plastic buckets. The overall objective of this study was to assess the effect of variations in type of fermentation vessel used and in the type of back-slopping on the $\mathrm{pH}$ drop and bacterial species composition during fermentation cycles. In case of fermentations in calabashes the effect of passive back-slopping, where backslopping occurs unintentionally through the transfer of bacteria via the inner surface of the vessel, was taken into account. Temperature, batch volume, and timing of the start of fermentation during the day were uncontrolled factors, which effects were investigated as well.

\section{The effects of uncontrolled factors}

The uncontrolled factors are interrelated. The time of day at which the fermentation was started had an implication for the temperature of the milk during fermentation. Temperature during fermentation is mainly dependent on the outside temperature; during the night the temperature of the milk drops below $18^{\circ} \mathrm{C}$ and during the day temperatures can rise to values above $30^{\circ} \mathrm{C}$. There are indications that fermentation vessel and the fermentation volume also had an influence on the temperature profile during fermentation. In calabashes we observed a lower maximum temperature compared to the maximum temperature found in buckets. As calabashes are plant material, the isolation and the ability for evaporation through the surface might be higher, which can buffer temperature changes of the milk. Also, the round shape of the calabashes might cause the sun to mainly warm the top of the calabash, which is not in contact with the milk, while the bottom, which had contact with the milk stays in the shadow. In case of the buckets, the sun can directly shine on the sides of the buckets, which are in contact with the milk. It seems that the colour of the buckets (white) did not help in reflecting enough sun to counter this effect.

\section{Acidification}

During all fermentation cycles, we observed a drop in $\mathrm{pH}$. The dynamics of this drop was not uniform among the fermentation cycles that received the same treatment, likely due to uncontrolled factors such as the exact timing of the start of fermentation during the day, as this depended on when the cows were milked.

Without active backslopping, acidification had a lag phase of 6 to 18 hours, both in buckets and calabashes. This variation in lag phase time could be caused by the time of the day the fermentation started. When active back-slopping was performed, we observed a strong decrease in lag time for acidification, not depending on the time of day the fermentation started. Due to the higher number of lactic acid producing bacteria present at the start of fermentation, the $\mathrm{pH}$ started to drop right after initiation of the fermentation process and continued during the night even when the temperature of the milk dropped. Without back-slopping the number of bacteria present in the milk is relatively low. As the temperature of the milk was going down after the start of fermentation, it took until the next day for lactic acid production to increase.

Fermentation in previously used calabashes showed great diversity in acidification rate. This is probably due to the inherent randomness of bacterial transfer from the biofilm 
of the calabash into the milk. For instance, the bacterial transfer can be dependent on the time between successive fermentations in the same calabash, the effectiveness of cleaning, the handling at the start of fermentation, and the physical properties of every individual calabash, such as its shape and the amount of cracks. This unpredictability of the fermentation process with passive back-slopping was also apparent in the variation in bacterial diversity over the course of fermentation. When a new calabash was used, a higher diversity was observed at the beginning of fermentation, probably due to a high influx of bacteria not associated with milk fermentation. However, the microbial community at the end of this fermentation showed a similar Shannon index compared the microbial communities of the other fermentation methods.

\section{Community diversity}

A diverse microbial community was found in traditionally produced Mabisi. Overall, the $\mathrm{pH}$ decreased over fermentation time, which made the environment generally more stressful for the microbial community. This resulted in a decrease of bacterial diversity, which is probably due to selection for only those bacteria that can grow in acidic environments. As the acidification rate in the different fermentation cycles varied, also the decrease in diversity showed variation. At the end point of fermentation there was no difference in diversity anymore. However, there is an indication for a trend towards a higher diversity in Mabisi from back-slopped fermentation in buckets compared to the other methods used here.

Lactococcus lactis was the bacterium with the highest presence at the end of all fermentations, between $47 \%$ and $99 \%$ of the total bacterial population. The remaining community had a very diverse character. Earlier research performed by Schoustra et al. (5) also found $L$. lactis as the main bacterial species present in the community. However, the number of different species found in the present study is higher due to the use of a different sequencing technique. In the study by Schoustra et al (2013), a high presence of Lactobacillus helveticus, Lactobacillus plantarum, and Acinetobacter ursingii was found; up to $20.4 \%, 14.9 \%$ and $20.0 \%$, respectively. In the current study, these species were only found in very low numbers; up to $0.4 \%, 0.03 \%$ and $0.09 \%$ of the total species abundance, respectively. These differences might be due to production methods of the Mabisi, sampling location, as well as methods of analysis. Unfortunately, the exact production method of the Mabisi analysed by Schoustra et al. (2013) is unknown because Mabisi samples were bought on markets. Also, a high initial growth rate of $L$. lactis might have caused it to enter the decline phase before the moment of analyses (157). If this is the case, it influenced the relative frequency of $L$. lactis compared to the other bacteria.

Community diversity can be a direct consequence of the number of niches present and the availability of different organisms. According to the niche exclusion principle $(104,105)$, niches can only be occupied by one species. Consequently, an environment with more niches is more likely to have a high diversity. A niche is defined by its physical characteristics, its chemical composition, and interspecies interactions in the total bacterial community (106). Due to their smooth surface, buckets are hypothesised to have less physical niches than calabashes, which have a rough inner surface. However, on average this study did not find a lower diversity in buckets. Both 
vessels used can have a sufficient number of niches to maintain a diverse community, due to various characteristics.

In the experiments conducted in this study, the buckets also had a larger opening than the calabashes, and this might have caused a higher influx of environmental microbes into the fermentation media before and during fermentation. Temperatures fluctuated more in buckets compared to the calabashes most likely due to differences in the opening dimensions and heat conductivity of the materials (see above). These fluctuations might increase the number of niches present, which could result in a more diverse community $(158,159)$. The milk in the containers was not mixed, increasing the possible heterogeneity in temperature and oxygen profiles, resulting again in a higher number of niches (113). Besides the availability of niches in the environment, also bacterial interactions can shape niches. More research towards community function of all species, for example by the analyses of the communities on an RNA level or reconstructing communities where hypothesised key-species are deliberately left out, might give insights in the cause of diversity. Diversity may also be explained in the context of niche specialization of species over time and competition. In the calabashes, species (co-)exist for prolonged periods of time, at least longer than when growing only for one fermentation cycle as is the case in the buckets. During the prolonged growth, species may specialize on the specific niche they occupy (124). Better adapted strains will outcompete the lesser adapted strains, resulting in a lower diversity in calabashes. However, when back-slopping is performed in buckets there is no such competition which can keep the diversity higher than in calabashes.

Overall an effect of the type of fermentation vessel used and the type of back-slopping on the bacterial diversity was not observed. These results do not match the trends found in recent surveys of Mabisi by Moonga et al (2017). Here, samples taken from Mabisi where some form of back-slopping was applied had a lower diversity than those without back-slopping. The fact that the back-slopping method is standard practice in that type of Mabisi might cause the difference from the current study. Repeated backslopping will put certain selection pressures on the bacteria which changes the species composition in the communities of the Mabisi. Further studies could show the effect of back-slopping on microbial diversity as well as on aroma profile and consumer preferences, for different types of Mabisi.

\section{Implications of microbial diversity}

Mabisi is a tasty and nutritious product and part of the cultural heritage. Therefore, understanding the microbial community can add to the production of a fermented product of high quality. The microbial community active during fermentation shapes the aroma profile of the final product as well as produces antimicrobial factors and prevents contamination.

Low $\mathrm{pH}$ and acidification rates have important implications for product safety. Products with a low pH are generally considered safe because this inhibits the proliferation of pathogenic bacteria (160). Faster rates of acidification might also influence product characteristics, such as taste and thickness (161). In terms of acidification, the spontaneous fermentation of Mabisi shows similarities with fermentations performed in controlled environments using starter cultures. Moreover, the decrease in diversity 
is comparable to, for example, that in the production of parmesan cheese, where an undefined starter culture is mixed with raw milk and the microbial community becomes less diverse during fermentation (12).

A diverse community is hypothesised to result in a product with a bigger variety of aroma components (162) and therefore potentially a richer taste. Also the chance of health beneficial bacteria to be present is higher for more diverse communities than for a product that only contains a few bacterial species (9). These bacteria can produce vitamins that are of importance to the human body or have a positive effect on the gut microbiome, which both are important for health. A more diverse community is also less prone to invasions of pathogens and bacterial spoilers $(100,163)$. This, in combination with a low pH might make Mabisi a microbial safe product. With bacterial diversity alone we could not substantiate the general believe that the Mabisi produces in calabashes is different from that produced in buckets. Likely, the variation of species in the communities cause this difference.

All the effects of diverse communities mentioned above are highly relevant for the producer of a fermented product such as Mabisi because one needs a good product for home consumption as well as a product one can sell for a good price. Compared to fermented products produced using simple starter cultures with only one on two strains, Mabisi might have a clear added benefit compared to these products, in terms of taste, nutritional value, and safety, because of its diverse bacterial community. Knowing which production steps have an influence on final product characteristics is therefore important for producers. This is true for Mabisi, but also for other products from spontaneous fermentation in general.

\section{Limitations}

In this study we maintained the process steps as close as possible to the traditional way of producing Mabisi. While this ensures that our study aligned with actual Mabisi practice resulting in a valuable overview of what Mabisi can be and how it can be produced, it also increased the number of uncontrolled and uncontrollable variables that may influence our ability to draw firm conclusions on the effect of our treatments on the acidification and microbial community dynamics. Moreover, the number of experiments that could be performed was highly dependent on the availability of raw milk and fermentation vessels. Clearly, this had implications for the statistical power of the described experiments. The indication that active back-slopping results in a higher diversity in buckets than in calabashes might be confirmed when expanding the experimental set-up.

It is likely that besides bacteria also yeasts and fungi are members of the fermenting community in Mabisi. However, as this is a non-alcoholic milk fermentation we choose to focus on bacteria, and particularly lactic and acetic acid bacteria, as these were hypothesised to contribute most to the fermentation of Mabisi. In future research, also the roles of other organisms besides bacteria should be investigated. 


\section{Conclusions}

While taking all uncontrollable variables in account, fermentation in the field showed overall great similarity to a controlled fermentation in terms of $\mathrm{pH}$ trajectory and microbial diversity at the end of fermentation as well as during fermentation. Bacterial communities in general decreased in diversity over time, where the drop in $\mathrm{pH}$, irrespective of variation in rate of acidification, correlated with a decreasing Shannon Index. No difference was found in microbial diversity during and at the end of fermentation performed in plastic buckets or previously used calabashes. Besides small differences, all processing methods resulted in a microbial community dominated by Lactococcus lactis and a Shannon Index, between 0.6 and 2.0. The use of plastic buckets for Mabisi fermentation can be a valuable alternative to the use of calabashes as this study showed no biological and physico-chemical differences between Mabisi resulting from both fermentation vessels, although the reason for perceived differences should be further investigated. These results are likely to have important implications for the flavour, microbial safety, and nutritional aspects of Mabisi. Certainly, these aspects should be further investigated to meet the needs of Mabisi producers and other related products.

\section{Acknowledgements}

This study would not have been possible without the collaboration and encouragement of members of Mondake dairy association and the people from the local community in Chibwe, Zambia. Special thanks go to Sarah Majuru, who hosted Anneloes Groenenboom during the study and helped with the coordination of the study in the local community. The involvement of all farmers from the Mondake Dairy association who contributed their milk and knowledge on Mabisi production is highly appreciated. Frank Phiri and Djondoh Sikalangwe are gratefully thanked for their help during the field experiments as well as other collaborators from Heifer International Zambia. Also, we thank our colleagues at the University of Zambia who helped with the preparation and laboratory work of this study. The help of Joost van den Heuvel in the analyses of the sequencing data is highly appreciated. Funding was received from NWO WOTRO Global Challenges Programme [W08.250.2013.108]. 


\section{Supplementary information}
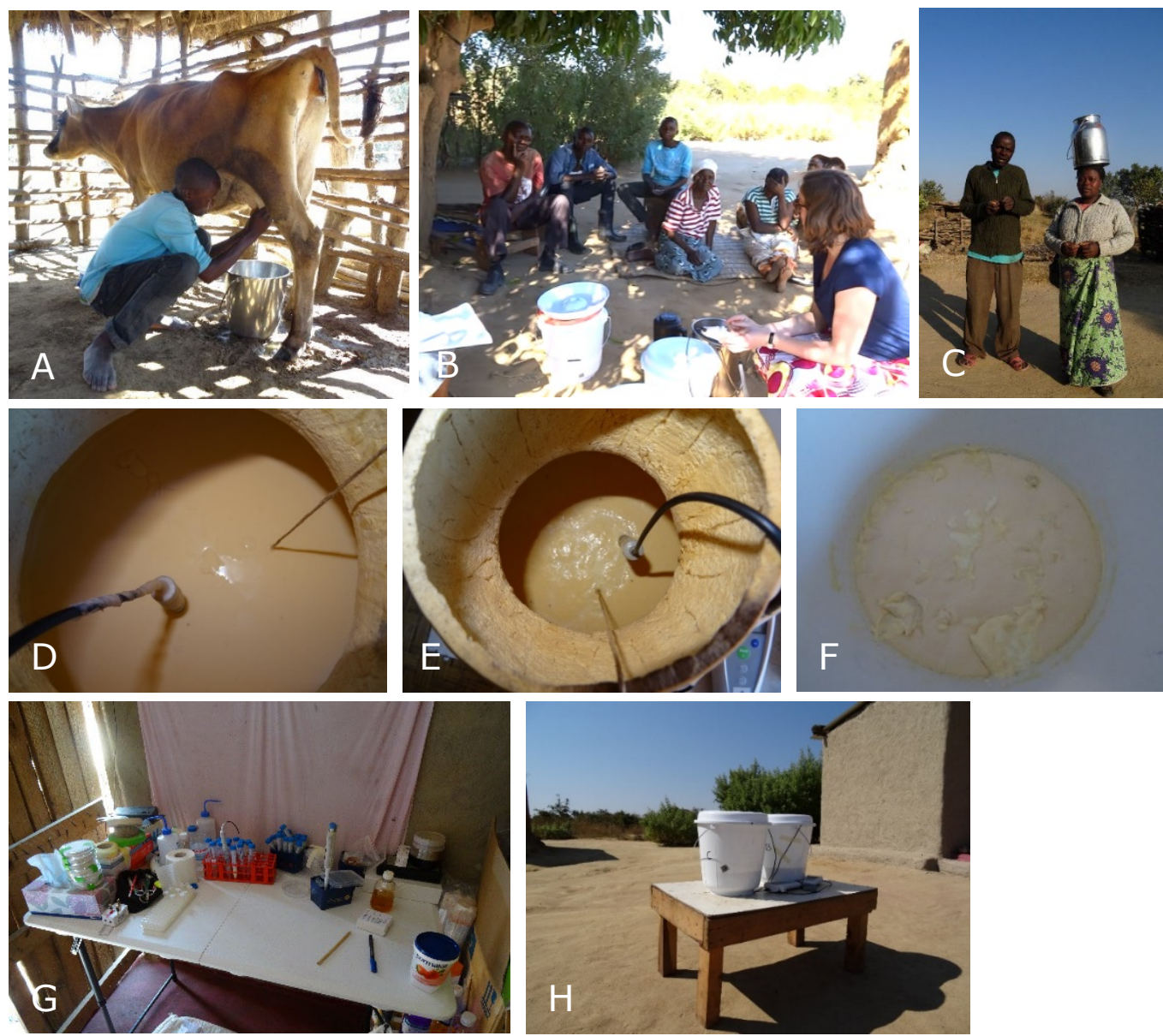

Supplement Figure 4.S1. Additional pictures of the field work. Panel (A) In the early afternoon the cows are milked for the second time that day. This milk was used for the fermentations described in this study. The time of milking differed per day and per farmer and was dependent on the other activities on the farm. As the milk was originating from different farmers, also the time between milking and 'start of fermentation' was variable. (B) During the experiments, aspects of the study, such as $\mathrm{pH}$ measurements, were explained to the interested local community. (C) Milk obtained from the morning milking was brought to the milk collection centre for shipment and sale to a nationwide operating dairy company. ( $D \& E$ ) Temperature and $\mathrm{pH}$ was monitored in the fermenting milk in the calabashes. Both temperature and pH probes were positioned in the centre of the fermenting liquid. (F) Fermented Mabisi in a bucket ready for mixing and consumption. Temperature and $\mathrm{pH}$ probes were removed and show thickening of the milk. (G) Field laboratory for performing simple analyses and sampling. Here the samples were transferred to the filter paper, the temperature and $\mathrm{pH}$ probes were cleaned. $(\mathrm{H})$ During the day all fermentation vessels were placed in the sun to allow temperature increase. 


\section{Supplement 4A: Information about Zambia and the importance of Mabisi}

In Zambia over 400 dairy cooperatives exist. The Mondake Dairy Association based in the rural part of Kabwe town in Zambia is a typical local farmer organisation. The Association expressed that local milk-processing and sales would present excellent opportunities for women entrepreneurship and financial self-reliance for women and their households. The Association has adopted an active policy towards improved gender equality and women's leadership.

Five years ago, the Association had around 100 members, of whom $20 \%$ were women. Currently, the membership has increased to 1274 and $60 \%$ are women. Members own 7 cows on average and milk 3 of their 7 cows, producing about 15 litres per day. Combined, the members of the Association produce 900 litres of milk per day. Around $75 \%$ of raw milk on a daily basis, especially the afternoon milk, is not collected at the milk collection centre by the commercial dairy company. The uncollected raw milk spoils and is discarded, leading to high postharvest losses at the very source of the milk value chain. Furthermore, the 900 litres are produced from milking only half of the available cows, as milking more cows would only increase the loss of fresh milk. Around $10 \%$ of milk is used to make Mabisi for household consumption, and excess Mabisi is sold to local customers. Fresh milk of grade $A$ sells at the local rural market or commercial milk collection centre, at 4 kwacha per cup (EUR 0.25) and Mabisi at 10 to 15 kwacha per cup (EUR 1 to 1.5 ).

Currently, there is no option to larger scale Mabisi production due to lack of facilities, training, and the lack of scaling up of technology. Members of the Association have been part of a project on traditional Mabisi. This project has generated basic knowledge on processing standards, on which the Association organised a workshop for its members jointly with the University of Zambia, Heifer International and Wageningen University. Furthermore, one of the members has visited the Netherlands for a twoweek workshop on dairy processing in the Netherlands, hosted at Wageningen University. This trip has been highly motivating and stimulating for the entire Association. The Cooperative is now looking into how to adopt new technology as well as develop skills in business development and marketing to transform themselves in a cooperative that can be an equal partner to other players in the value chain of agricultural produce. 


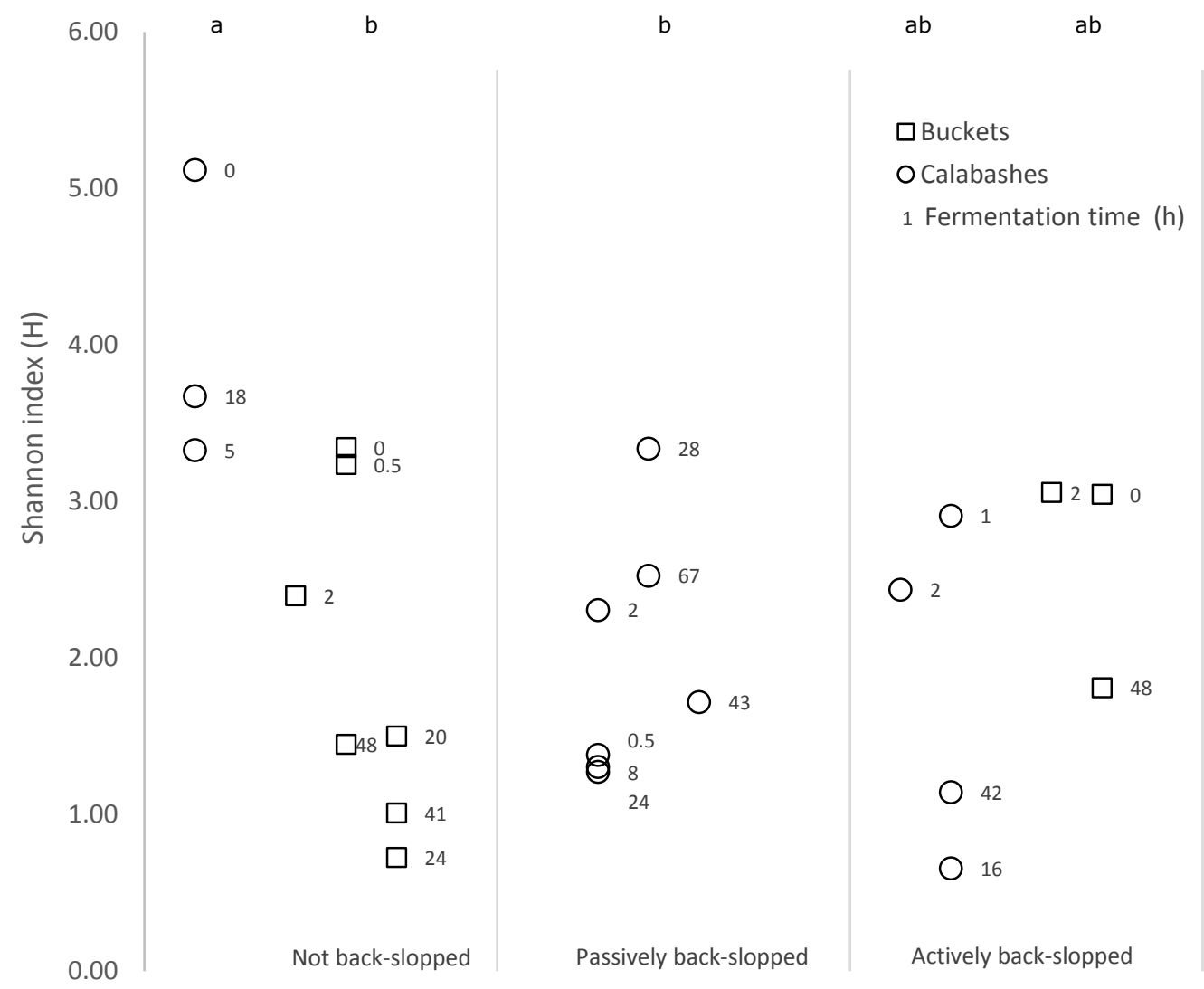

Supplement Figure 4.S2. Shannon index based on OTUs as a measure for bacterial diversity during fermentations in buckets $(\square)$ and calabashes $(\circ)$ with and without back-slopping. Number next to data points indicate time after start of fermentation in hours. Different fermentations from one processing method presented next to each other. Letters above the columns indicate significant difference at the 0.05 level taking all samples from one method together. Some samples could not be included because the field experiment did not allow regular sampling and some samples were lost due to contamination. 
Chapter 5. 


\title{
Does change in bacterial species composition of natural communities reflect adaptation to a new environment?
}

\author{
Anneloes E Groenenboom ${ }^{a, b}$ \\ Joost van den Heuvela
}

Bas J Zwaan ${ }^{a}$

Eddy J Smid ${ }^{\mathrm{b}}$

Sijmen E Schoustraa,c

\footnotetext{
a Laboratory of Genetics, Wageningen University and Research, Wageningen, The Netherlands b Food Microbiology, Wageningen University and Research, Wageningen, The Netherlands

c Department of Food Science and Nutrition, University of Zambia, Lusaka, Zambia
} 


\section{Abstract}

Background: Microbial ecosystems generally consist of communities with a complex composition. Repeated propagation cycles in a new environment can change species composition. Various factors, such as initial species composition and pressure from the environment, may influence this change.

Research questions: What happens when natural bacterial communities of different origin are brought in a novel, yet similar environment? Will comparable communities converge to the same composition, will they remain different or become even more different from each other over time, and what factors drive these changes?

Approach: Six comparable but different natural microbial communities were each split into five identical replicates, which underwent 16 repeated propagation cycles in a novel, common environment. We compared bacterial species composition at the start and the end of the experiment. The influence of four main factors on species composition, i.e. initial composition, impact of the environment, changes due to interaction between species, and random processes in species dynamics, was tested by modelling hypothetical species compositions. The observed and modelled species composition were compared to infer the driving factors behind this its dynamics.

Conclusion: Replicate communities that are from the same ancestral community changed in a similar way in response to the new environment. At the end of the repeated propagation cycles, communities were either dominated by Lactobacillus helveticus or by Lactobacillus delbrueckii. Initial species composition seemed to determine the direction of development, followed by random processes. The nature of the environment had the least influence on the change in species composition.

\section{Introduction}

In natural ecosystems various species co-exist thus forming communities. Species composition describes what species are present and the diversity of these species, in terms of number and distribution. Similar ecosystems have similar species diversity, for instance in the species diversity of Darwin's finches on similar islands and species diversity of cichlid fish in African lakes, showing that eco-systems are relatively stable in terms of their species diversity $(37,39)$. Biotic and abiotic factors are thought to stabilise eco-systems (104) and species composition can change due to selection pressures $(55,76,164,165)$. Selection pressures may shift the balance among the coexisting species favouring the species which are best adapted to the selection pressure, leading to a process of species sorting (166). Key questions in species sorting include whether species sorting would lead to parallel or divergent change when these communities encounter the same change in environment, given that similar species are present in ancestral communities. Also, analysing the level of repeatability of species sorting could give insights in how ecosystems are constructed (40).

Here we study how a change of environment can change species composition in a microbial eco-system derived from Mabisi. Mabisi is a product of spontaneous fermentation of milk, which is traditionally produced in Zambia and of which the bacterial community consists of six to ten main species of lactic acid and acetic acid 
bacteria (5). Production methods of Mabisi differ per region (8); in most cases, raw milk is filled in a container, either a calabash, bucket or milk can, and left undisturbed for 24 to 48 hours after which it is stirred and consumed. The resulting community is re-used for the production of the next Mabisi by addition raw cow milk to the containers in which the community is present. It is interesting that these communities have been co-cultured up to tens of years or maybe even more. In a food technology context the serial transfer of material is referred to as backslopping (13).

In this experiment six original Mabisi samples were used. Species composition of each sample is different, yet very similar. We characterized the samples in terms of their species composition at the start of the experiment and after 16 repeated transfers in a common environment. The central question we address is whether initially similar communities will either become more alike (convergence) or less alike (divergence) with respect to their species composition. Final species composition could be affected by the initial composition (history - species present and their relative abundance) and the selective pressures during the repeated transfers imposed by the environment (change). If the fact that we place communities in a new, slightly different from the original, environment is the main driver of change in species composition, the communities will become more alike. However, should the slight differences in species diversity between the six original Mabisi samples be the main driver, species diversity may diverge. By using five replicates of each of the six original Mabisi samples for the repeated transfers, we will assess how repeatable changes in species composition are when starting with communities with slight initial difference in species composition and place them in a new environment. This will show whether there would be an optimum in species composition in a given environment. This selection at the level of species is comparable to the process of natural selection acting on standing genetic variation within one species.

Two traits related to community dynamics, metabolic profile and species composition, are measured at the beginning and end of the experiment. We used a custom statistical model to test whether initial community composition and environment were significantly affecting community dynamics and if so, whether this happened according to an additive or interactive scenario. For this we used a log-likelihood ratio test with multinomial probabilities distributions.

\section{Materials and methods}

\section{Mabisi samples}

For these experiments, fermented milk products from Zambia were collected. This product, called Mabisi, was purchased on the market of Mumbwa, Kaoma, and Nangoma in February 2015. In Mumbwa, Mabisi was bought from three producers, and one producer sold two types of Mabisi. This resulted in six Mabisi products: four from Mumbwa, one from Kaoma and one from Nangoma. Upon collection, the samples were stored in the fridge and shipped to the Netherlands at ambient temperatures.

\section{Repeated propagation cycles}

After arrival in the laboratory the bacterial communities were incubated in $30 \mathrm{~mL}$ UHT milk (Milbona, Lidl) with $1 \mathrm{~mL}$ of Mabisi. The incubation period was 3.5 days at $27^{\circ} \mathrm{C}$. 
The resulting communities (considered T0) were used to inoculate the experiments. Five lines (replicates) were incubated per original Mabisi sample, $750 \mu \mathrm{L}$ Mabisi in 75 $\mathrm{mL}$ UHT milk, resulting in 30 lines. Also two blank UHT milk lines were transferred, but not initially inoculated. Every 3.5 days, $750 \mu \mathrm{L}$ of the fermented milk was transferred to $75 \mathrm{~mL}$ UHT milk, $\mathrm{pH}$ was measured and samples $(1 \mathrm{~mL})$ were taken for DNA extraction to allow full community profiling. In total 16 transfers were made, resulting in an average of 106 generations, assuming the bacterial diversity could increase a hundred fold after the dilution step during transfer $(\log (100) / \log (2)=6.64$ generations per propagation cycle, gives $6.64 * 16=106$ generations in total).

\section{DNA extraction}

The DNA extraction method was adapted from Ercolini et al. and Schoustra et al. $(5,140)$. For DNA extraction, $1 \mathrm{~mL}$ of fermented milk was spun down ( 2 minutes, 12000 RPM), after which the supernatant was removed. The cells were re-suspended in a mix of $64 \mu \mathrm{L} \operatorname{EDTA}(0.5 \mathrm{M}), 160 \mu \mathrm{L}$ Nucleic Lysis Solution, $5 \mu \mathrm{L}$ RNAse, $120 \mu \mathrm{L}$ lysozyme and $40 \mu \mathrm{L}$ pronase $\mathrm{E}$. After an incubation time of 60 minutes at $37^{\circ} \mathrm{C}$ and agitation of $350 \mathrm{RPM}, 400 \mu \mathrm{L}$ ice-cold ammonium acetate (5 M) was added and the mixture was cooled on ice for 15 minutes. The mixture was spun down and $750 \mu \mathrm{L}$ of supernatant was transferred to a tube containing $750 \mu \mathrm{L}$ phenol. This tube was vortexed and its content spun down ( 2 minutes, $12000 \mathrm{RPM}$ ) and $500 \mu \mathrm{L}$ of supernatant was transferred to a tube containing $500 \mu \mathrm{L}$ chloroform. This tube was vortexed and its content spun down ( 2 minutes, $12000 \mathrm{RPM}$ ) and $400 \mu \mathrm{L}$ of supernatant was transferred to a tube containing $1 \mathrm{ml} 100 \%$ ethanol and $40 \mu \mathrm{L}$ sodium acetate $(3 \mathrm{M})$. This DNA containing tube was left to precipitate at $-20^{\circ} \mathrm{C}$ overnight. The next day, the tube was spun for 20 minutes at $12000 \mathrm{RPM}$ at $4^{\circ} \mathrm{C}$. The supernatant was carefully aspirated, and the DNA pellet was washed by adding $1 \mathrm{~mL} 70 \%$ ethanol. The tube was spun for 10 minutes at 12000 RPM at $4{ }^{\circ} \mathrm{C}$, after which the supernatant was aspirated again. The DNA pellet was left to dry at room temperature and dissolved in $20 \mu \mathrm{L} 10 \mathrm{mM}$ Tris pH 7.5.

\section{Bacterial community profiling: Species composition}

The 36 extracts ( 6 original Mabisi and $6 * 5$ of samples at time point 16) containing DNA from all organisms in the community was sent for bacterial 16S rRNA gene amplicon paired-end sequencing of the V4 hypervariable region (341F-785R) on the MiSeq Illumina platform by LGC genomics (Berlin, Germany).

For further data processing and statistics the QIIME pipeline (141), modified from Bik et al (142), was used. Paired-end reads were joined using join_paired_ends.py (with minimum overlap 10 basepairs) after which sequences were trimmed and filtered using cutadapt (v1.11 -q 20, -m 400, Martin 2011) using the known primer sequences CCTACGGGNGGCWGCAG and GACTACHVGGGTATCTAAKCC. These trimmed sequences were then checked for chimera's, using uchime (v4.2.20, gold database)(155), with sequences with a lower chimera score than 0.28 were retained. After these trimming and filtering steps sequences were clustered into operational taxonomic units (OTUs) after quality check using pick_open_reference_otus.py (-s 0.1 , enable_rev_strand_match TRUE, -align_seqs_min_length 75, -pick_OTU_similarity 0.95). Taxonomy of the resulting OTUs was assigned to representative sequences using the Greengenes (v13.5) rRNA database. This algorithm gives a representative 
sequence for an OTU, which were used to perform a local blast using the gold database from uchime. The taxonomy from the top BLAST hit was used for further data processing. Shannon index $(\mathrm{H})$ accounts for both number and evenness of OTUs present and is calculated using:

$H=-\sum_{i=1}^{S}\left(p_{i} \ln p_{i}\right)$

in which $\mathrm{p}_{\mathrm{i}}$ is the proportion of reads belonging to category $\mathrm{i}$, and $\mathrm{s}$ is the total number of categories which can be OTUs, species, or genera depending on the level of clustering of the reads. The Shannon index can lie between 0 and In s (156), depending on the distribution of the categories (evenness).

A second sequencing run was performed including intermediate time points. Unfortunately, we experienced that the first batch of next generation sequencing could not be compared to the second batch. Samples that were sequenced in both batches showed much variation in species diversity and evenness. Also, when clustering the samples in a PCA, the two batches of sequencing clustered together leaving no room to interpret the data in a combined way. Other studies have experienced the same $(142,167)$ and the exact reasons for this technical artefact are unknown.

\section{Bacterial community profiling: Metabolic profile}

Frozen samples from time points 0 and 16 were defrosted for volatile metabolites profile analyses using GC-MS. After an incubation of $20 \mathrm{~min}$ at $60{ }^{\circ} \mathrm{C}$, volatiles were extracted using a SPME fibre (Car/DVB/PDMS, Suppelco) for $20 \mathrm{~min}$ at $60^{\circ} \mathrm{C}$. Volatiles were desorbed from the fibre for 2 minutes on a Stabilwax- DA-Crossbond-Carbowaxpolyethylene-glycol column (30 m length, $0.25 \mathrm{mmID}, 0.5 \mu \mathrm{m}$ df), PTV Split-less mode (5 minutes) at $250^{\circ} \mathrm{C}$, helium as carrier gas at $1.5 \mathrm{~mL} / \mathrm{min}, \mathrm{GC}$ over temperature at $40^{\circ} \mathrm{C}, 2 \mathrm{~min}$, raised to $240^{\circ} \mathrm{C}\left(10^{\circ} \mathrm{C} / \mathrm{min}\right)$ and kept at $240^{\circ} \mathrm{C}$ for $5 \mathrm{~min}$. Mass spectral data was collected over a range of $\mathrm{m} / \mathrm{z} 33-250$ in full scan mode with 3.0030 scans/seconds. Results were analysed with Chromeleon 7.2 CDS Software (ThermoFisher) where 32 signal peaks were identified as volatile metabolites according to their elution time and mass spectral data.

\section{Statistical Modelling}

To test whether and how the initial species composition impacts the change in species composition upon propagation we employed a statistical modelling approach. In this model we related the initial (TO) and end composition (T16) by a vector of transformation values that link these two communities. The transformation values link T0 and T16 composition as follows,

$O T U_{i, j, k, T 16}=O T U_{i, j, T 0} w_{i, j, k} / W$

where OTU $\mathrm{O}_{\mathrm{i}, j, k, T 16}$ is the frequency of $i$ th OTU from original Mabisi sample $j$ at timepoint $\mathrm{T} 16$, measured for the $k$ th replicate. The transformation values $\mathrm{w}_{\mathrm{i}, \mathrm{j}, \mathrm{k}}$ determine whether an OTU will increase or decrease. Note that the total sum of the frequencies was scaled by factor $W$ such that the total frequency of OTUs becomes 1 . We modeled five scenarios (S1-5); the transformation values were dependent on initial species composition (S1), time (S2), initial species composition + time (S3), the interaction 
between initial species composition and time (S4), and the effect of the interaction of initial species composition and time + stochasticity (S5). We compare these scenarios to a null hypothesis, where there are no differences between any samples (S0).

We determined the probability of each scenario by calculating the likelihood of sampling an OTU table $\left(S_{i, j, k, T 16}\right.$ and $\left.S_{i, j, T 0}\right)$ from the OTU table frequencies (OTU $U_{i, j, k, T 16}$ and $\left.\mathrm{OTU}_{\mathrm{i}, j, \mathrm{TO}}\right)$. To render it possible to calculate the likelihoods of the distributions, we used a rarefaction in qiime to obtain $S$ (rarefaction.py, $-\mathrm{m} \mathrm{100)}$ ). The probability of finding $S$ from a distribution of OTU frequencies is determined using the multinomial distribution function dmultinom() in $\mathrm{R}$ ( $\mathrm{R}$ base) for each of the 36 samples. From all possible OTU models we have taken the one with the highest likelihood, given the modeled scenario. The likelihood would be highly dependent on T16 samples as for each original Mabisi sample there are five times more propagated samples compared to non-propagated (TO) samples. Therefore, we weighted the log-likelihood of each of these samples by dividing it by 5 . We let $P(S ; O T U)$ be the probability that we sample $S$ out of frequency distribution OTU. Then the log-likelihood summed over all samples of a scenario is given by,

$L=\sum_{j=1}^{6} \ln \left[P\left(S_{j, T 0} ;\right.\right.$ OT $\left.\left._{j, T 0}\right)\right]+\frac{1}{5} \sum_{k=1}^{5} \sum_{j=1}^{6} \ln \left[P\left(S_{j, k, T 16} ;\right.\right.$ OT $\left.\left.U_{j, k, T 16}\right)\right]$

The frequency distribution with highest likelihood could be determined in all but one scenario, by averaging the frequencies found; i.e., the most likely scenario for frequency is the average frequency. For instance, for SO, where the scenario is that of no difference in distribution between any samples, the most likely OTU distribution is the average over all the OTU frequencies, i.e.,

$O T U_{M L, S 0}=\frac{1}{12}\left[\sum_{j=1}^{6} O T U_{j, T 0}+\frac{1}{5} \sum_{k=1}^{5} \sum_{j=1}^{6} O T U_{j, T 16}\right]$

In the second scenario we model the potential effect of initial species composition, without an effect of propagation (S1). The most likely OTU frequency distribution is the average over the OTU tables within a sample coming from one original Mabisi sample.

$O T U_{M L, S 1, j}=\frac{1}{2}\left[O T U_{j, T 0}+\frac{1}{5} \sum_{k=1}^{5} O T U_{j, k, T 16}\right]$

Third, we model only the effect of time point (S2), where the most likely distribution is that of the mean over all OTU frequencies within a timepoint. Therefore, this is

$O T U_{M L, S 2, t}=\overline{O T U_{t}}$

where $t$ stands for different timepoints, which can be T0 and T16. Then we modeled the effect of both initial species composition and time, but with similar changes in time for each original Mabisi sample (S3), i.e, $w_{i, 1, k}=w_{i, 2, k}=\ldots . w_{i, 6, k}$.

In this scenario, an evolutionary algorithm was used to find the most likely frequency distribution, as a similar analysis of averaging (eqs. 3-6) could not lead to the most likely distribution. This scenario started with the measured OTU tables frequencies at TO as the starting modeled OTU frequencies. Then we started all values of $w$ to be the 
mean values of OTU at T16 divided by T0, scaled in a similar way as in eq. [1]. Subsequently we varied the values for OTU at TO and all values for $w$ between generations by multiplying these by a random number taken from a normal distribution with mean 1 and standard deviation of a uniform distribution that varied between 0 and 0.05 . Therefore, nor the steps, nor the step size were equal every generation. For the obtained values of most likely OTU distribution eq. 2 was calculated and when this likelihood was higher than before, the values obtained in the current run became the newly inherited parameter values for OTU T0, OTU T16 and all values for $w$. This simulation was run for 10,000,000 generations, within which the most likely OTU values showed asymptotic behavior.

Then we modelled the scenario in which initial species composition affected how OTU frequencies changed during propagation (i.e., interaction, S4), which was again estimated by taking the mean of the OTU tables, but now per time point per original Mabisi sample. This was calculated using

$O T U_{M L, S 4, j, t}=\overline{O T U_{J, t}}$

Lastly, when we also allowed for stochastic variation (S5), each most likely OTU table is the sampled table.

Once we had obtained the likelihoods of the most likely distributions, we tested whether initial species composition significantly affected OTU tables by performing a log likely ratio test between S1 and S0. Similarly, we performed such tests between the likelihoods for S0 and S2 (for propagation effect), the interaction between initial species composition and propagation (S3 vs S4) and lastly whether stochasticity significantly affected the OTU tables by comparing S4 and S5. The value obtained was tested against $\mathrm{X}^{2}$ distribution with difference in parameters as degrees of freedom.

Then we also quantified the relative explaining power for the different factors by calculating McFaddens Pseudo $R^{2}$ (168). We first calculated these values by

$R_{S i}^{2}=1-\log \left(L_{S i}\right) / \log \left(L_{S 0}\right)$

The maximum $R^{2}$ is obtained from $R^{2}$ s5. However, this value is still below 1 . To calculate the relative contribution of explanatory power we divided all values of $R^{2}$ by the highest $R^{2}, R^{2} s 5$, thereby getting $r R^{2}$, the relative McFaddens pseudo $R^{2}$. Lastly, to obtain the added $r R^{2}$ of single factors, such as stochasticity, we had to subtract the value of $r R^{2} s 4$. We thereby got the relative contribution for each factor.

\section{Results}

\section{Species composition}

The experiment consists of six initial Mabisi samples. Upon analyses of the microbial communities of these Mabisi samples 461 different OTUs were identified which blasted as 47 different species in two main genera, Lactobacillus and Acetobacter (Figure 5.1). In two samples, those originating from Kaoma and Nangoma, $61-64 \%$ of the reads blast as Acetobacter species and $30-35 \%$ as Lactobacillus species. In the other four samples, that originate from Mumbwa, the microbial communities consisted for about 
$60-70 \%$ of Lactobacillus and $30-40 \%$ Acetobacter species. The main species present were Lactobacillus helveticus, Lactobacillus delbrueckii, Acetobacter pasteurianus and Acetobacter orientalis. Lactobacillus fermentum and Lactobacillus kefiri are present in lower amounts and not in all samples. Shannon's diversity index of the samples ranged from 1.75 (Mabisi 4) to 2.11 (Mabisi 3) (Supplement Table 5.S1).

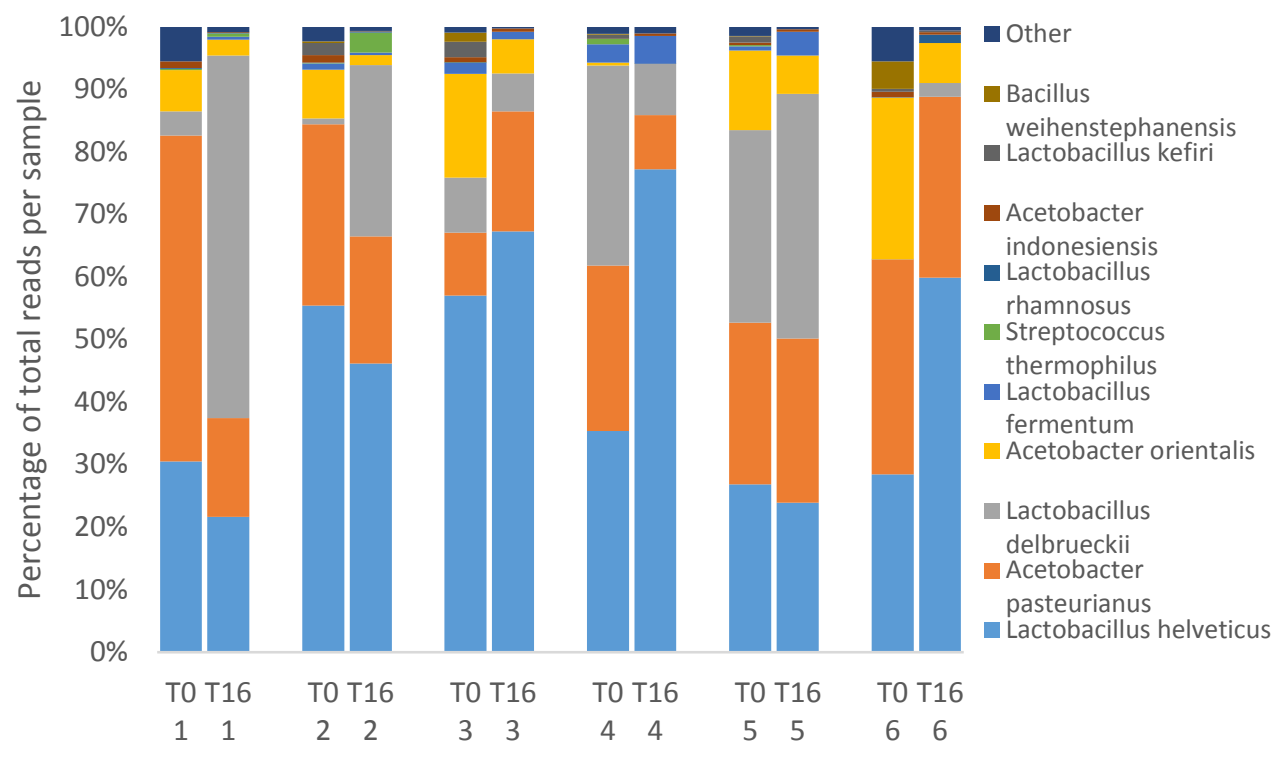

Figure 5.1 The microbial community structure of Mabisi samples at time point 0 and the average microbial community structure of the samples after 16 repeated propagation cycles (time point 16). Results of DNA sequencing in the $16 \mathrm{~S}$ region and shown on species level. Results shown for the samples after 16 repeated propagation cycles are the average of 5 replicates that were propagated independently. Microbial community structure of all 5 replicates can be found in Supplement Figure 5.S1.

The six Mabisi samples were split in five replicates and inoculated in a new milk environment. After $48 \mathrm{~h}$ of growth, we transferred $1 \%$ of the culture consisting of the mixed bacterial community to fresh milk and repeated the procedure for 16 repeated transfers. After 16 transfers the bacterial community structure was analysed again. The propagated Mabisi communities show differences from the original propagated samples in terms of species composition (Figure 5.1). On average the relative abundance of Lactobacillus delbrueckii and Lactobacillus helveticus increased while both Acetobacter pasteurianus and Acetobacter orientalis decreased in relative abundance. There is variation between groups of propagated lines. In propagated communities originating from Mabisi sample 4, for example, the abundance of Lactobacillus delbrueckii decreases instead of increases. Across all 5 propagated replicates that originated from this Mabisi sample (Supplement Figure 5.S1), this is a parallel change. Also, for the other replicates originating from one original community a parallel change can be seen. In most cases, except among propagated communities originating from Mabisi 5, the Shannon index was lower after propagation (T16) than before propagation (T0) (Supplement Table 5.S1). 
We used a principle component analysis to visualize shifts in species composition after repeated propagation cycles (Figure 5.2). This analysis shows that species composition in the five replicates originating from the same original Mabisi sample changed in a similar way. While replicates coming from the same Mabisi sample still show much similarities, the bacterial community structure over all the propagated microbial communities became less similar. Overall, species composition among the propagated communities shows a higher variability than overall species composition among the six starting communities. Two clusters are formed, representing two ecological states. One with Mabisi 1 and 5 (cluster A) and one with Mabisi 2, 3, 4 and 6 (cluster B). Compared to cluster $B$, cluster $A$ is characterized by a decrease in relative abundance of Lactobacillus helveticus and higher relative abundance of Lactobacillus delbrueckii.

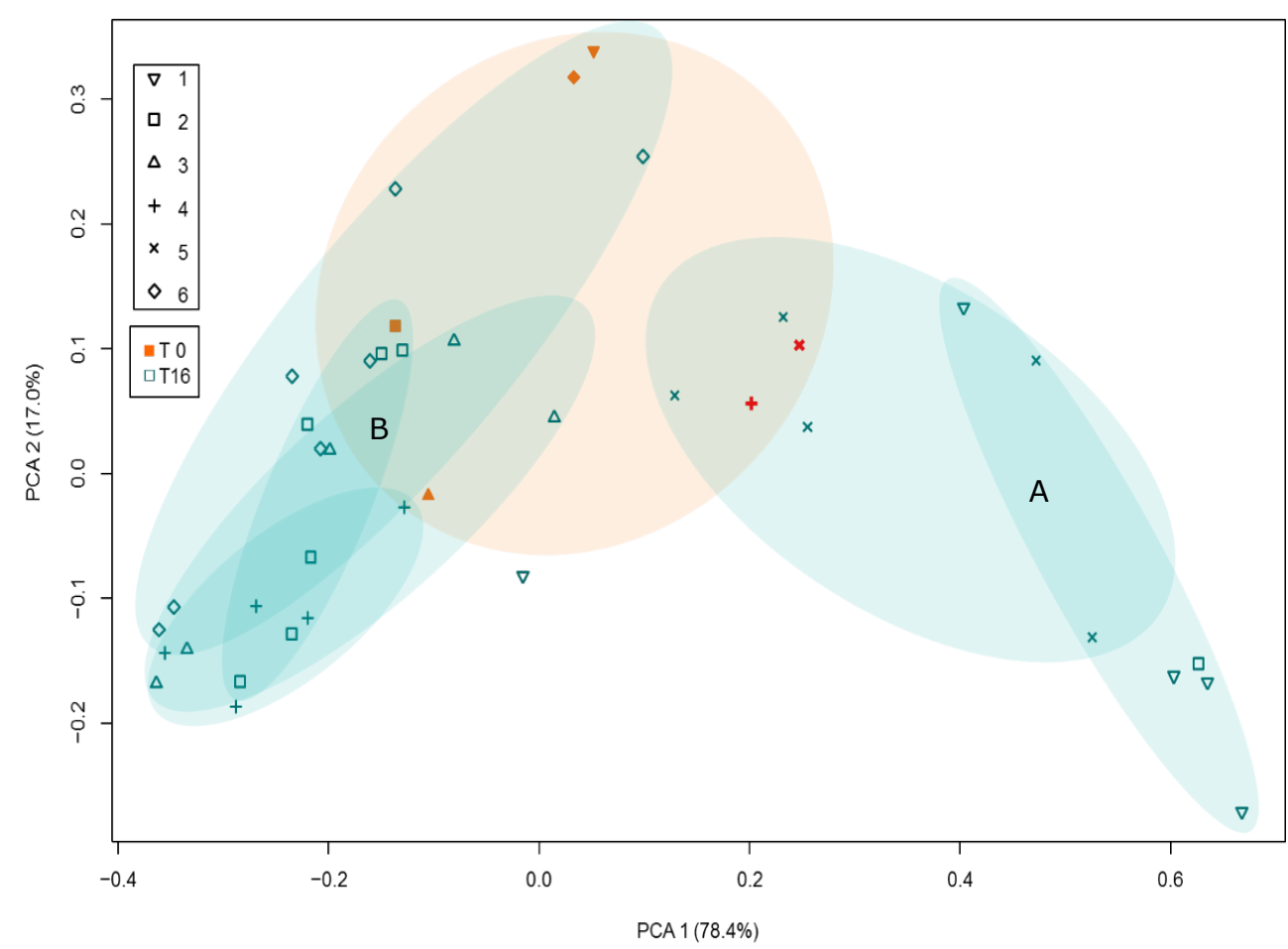

Figure 5.2 Principle component analyses (PCA) of bacterial community structure of all samples at T0 (orange) and T16 (blue). Shapes represent samples originating from the same original Mabisi sample. Oval orange shades show clustering of T0 samples, blue shades show clustering of T16 samples that had originated from the same T0 sample. Oval shades do not represent results of analysis and are for visual interpretation purposes. Two ecological clusters were observed among the T16 samples: cluster A characterized by a higher relative abundance of Lactobacillus delbrueckii, and cluster B characterized by a higher relative abundance of Lactobacillus helveticus. PCA analyses were based on OTU tables. 


\section{Metabolic profiles}

For overall metabolic activity, 32 volatiles were analysed as proxy. Analysing volatiles is a rapid and simple methods that, even though non-volatile metabolites are not analysed, gives us the possibility to compare metabolic activity between the different samples. Most volatiles belonged to the groups of esters, carbolic acids, ketones, and alcohols (Supplement Table 5.S2). We used a principle component analysis to visualize changes in metabolite profiles of communities before and after repeated transfers. In this case, the PCA analysis of the results did not show a clear distinction in clusters (Figure 5.3). Most samples, except for samples from Mabisi 6 show very similar volatile composition which is not, or only slightly, different from the volatile composition at T0. Other community characteristics such as $\mathrm{pH}$, phase separation, and product thickness also did not show a directional change (data not shown).

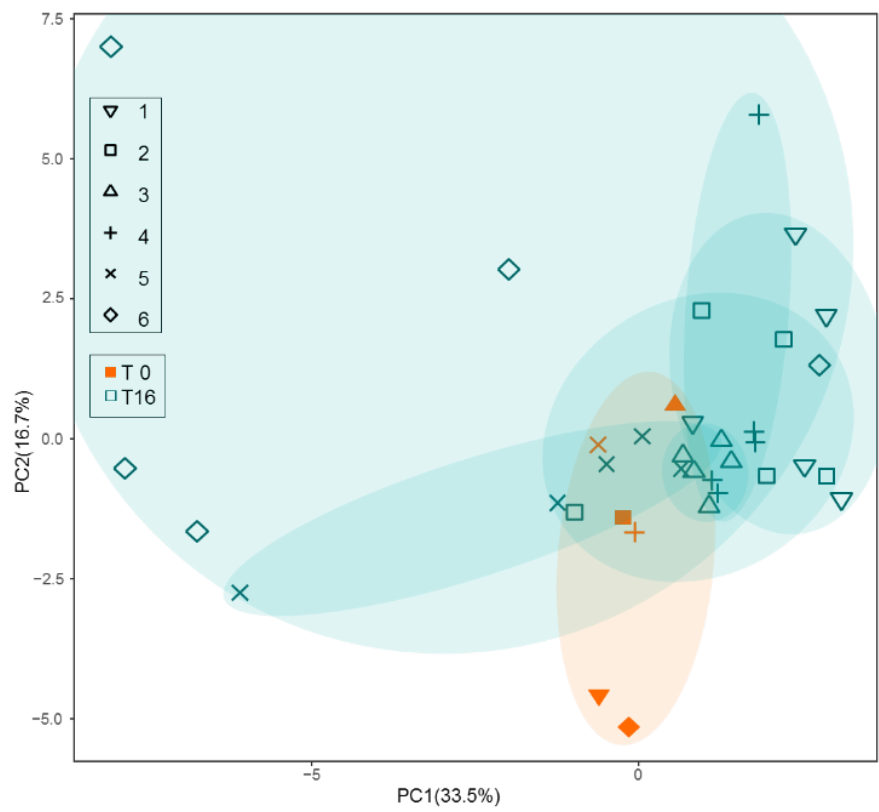

Figure 5.3 Principle component analyses of volatile compounds of all samples at T0 (orange) and T16 (blue). Similar shapes represent samples originating from the same original Mabisi sample. Oval orange shades show clustering of T0 samples, blue shades show clustering of T16 samples that had originated from the same T0 sample. Oval shades do not represent results of analysis and are for visual interpretation purposes. PCA analyses was based on the 32 most abundant volatiles in the GC-MS analyses as mentioned in Supplement Table 5.S2.

\section{Mechanisms that determine changes in species composition}

Changes in species composition after repeated propagation cycles can be due to four main factors: initial species composition, selection imposed by the environment, change caused by interaction between species, and random processes in species dynamics. To statistically test which factor has the biggest influence on the community structure, we developed a maximum likelihood approach where we add levels of 
complexity to a species composition model. Using the species composition of the 36 samples we modelled six different scenarios and calculated the log likelihood (Table 5.1). Visual representation of this step-wise model on species composition is in Supplement Figure 5.S2.

Every step increases the log likelihood. Allowing differences between the six initial samples (S1, Initial species composition) explains most of the variation (46.5\%) and significantly affected the species composition $\left(X^{2} d f=60=396.03, p<0.001\right.$, Table 5.2). While time also significantly affected the species composition (12.0\% of the variation explained, $\mathrm{X}^{2} \mathrm{df}=12=101.87, \mathrm{p}<0.001$ ), allowing for interactions between Mabisi sample and time points (Initial species composition * Time) explains $18.6 \%$ more of the variation then without interaction (Initial species composition + Time, $X^{2} \mathrm{df}=6=158.20$, $\mathrm{p}<0.001$ ). The addition of stochasticity (Initial species composition * Time + Stochasticity) resulted in explaining $100 \%$ of the variation, as all possible sources of variation are incorporated, however due to the many parameters included in this model (d.f. $=288$, Table 5.2), the addition of stochasticity did not significantly explain more of the OUT table variation $(p=1.0)$.

Table 5.1 Log likelihood of scenarios in species composition. The description of the scenarios and log likelihood are listed. OTU cutoff 0.02 , likelihood of time point 16 samples averaged. Input = rarefaction_100_1.biom. 100000 iterations for estimating S3.

\begin{tabular}{|c|c|c|}
\hline SCENARIO & DESCRIPTION & $\begin{array}{l}\text { LOG } \\
\text { LIKELIHOOD }\end{array}$ \\
\hline SO - ALL EQUAL & $\begin{array}{l}\text { No initial differences, } \\
\text { No composition changes over time }\end{array}$ & -562.18 \\
\hline $\begin{array}{l}\text { S1 - INITIAL SPECIES } \\
\text { COMPOSITION }\end{array}$ & $\begin{array}{l}\text { Initial differences, } \\
\text { No composition changes over time }\end{array}$ & -364.16 \\
\hline S2 - TIME & $\begin{array}{l}\text { No initial differences, } \\
\text { Equal composition changes over time }\end{array}$ & -511.24 \\
\hline $\begin{array}{l}\text { S3 - INITIAL SPECIES } \\
\text { COMPOSITION + TIME }\end{array}$ & $\begin{array}{l}\text { Initial differences, } \\
\text { Equal composition changes over time }\end{array}$ & -310.83 \\
\hline $\begin{array}{l}\text { S4 - INITIAL SPECIES } \\
\text { COMPOSITION * TIME }\end{array}$ & $\begin{array}{l}\text { Initial differences, } \\
\text { Different composition changes over time }\end{array}$ & -231.73 \\
\hline $\begin{array}{l}\text { S5 - INITIAL SPECIES } \\
\text { COMPOSITION } * \text { TIME + } \\
\text { STOCHASTICITY }\end{array}$ & All samples at T16 a different distribution & -134.35 \\
\hline
\end{tabular}


Table 5.2. Statistics of maximum likelihood approach using likelihood ratio tests. The log likelihood ratio test statistic $(2 * \Delta L L)$, number of degrees of freedom and $p$ value (from chi square distribution) and relative McFadden pseudo R2 are listed. OTU cutoff 0.02 , likelihood of time point 16 samples averaged. Input = rarefaction_100_1.biom. 100000 iterations for estimating S3.

\begin{tabular}{l|l|l|l|l}
$\begin{array}{l}\text { SCENARIOS } \\
\text { TESTED }\end{array}$ & $\begin{array}{l}\text { LR TEST } \\
\text { STATISTIC }\end{array}$ & AD.F. & P & REL. MCF PSEUDO R \\
\hline S1 VS S0 & 396.03 & 60 & 0 & $46.5 \%$ \\
S2 VS S0 & 101.87 & 12 & $6.7 \mathrm{e}-16$ & $12.0 \%$ \\
S4 VS S3 & 158.20 & 6 & $9.8 \mathrm{e}-10$ & $18.6 \%$ \\
S5 VS S4 & 194.75 & 288 & 1.000 & $22.9 \%$
\end{tabular}

\section{Discussion}

\section{Changes in species composition and metabolic profile}

We used six Mabisi samples from Zambia as starting point for repeated propagation cycles into fresh milk, splitting each original sample into five replicates. At the genus level, the six original microbial communities show much similarity, however, when comparing the communities at the level of species and OTUs, differences between the communities can be observed (Supplement Figure 5.53). The level of similarity at the genus level, in combination with a variation in OTUs, made the different bacterial communities a suitable starting point to study potential change in species composition upon repeated propagation cycles. The original communities harboured similar species (although each species could be present at a different frequency) and thus contained the potential to become the same. The environment used for the repeated propagation cycles is simpler than the Mabisi environment the bacterial communities originated from. The new environment might have been selective for only those bacteria that were most fit for this specific environment. This potentially caused a loss in species diversity towards retaining only a few species in the microbial community. This is however not what we observed. The milk environment appeared to provide enough niches to the bacterial species to maintain species diversity.

We observed a high level of parallel changes in species composition among the replicates of the same initial sample. Some divergence was observed, but in comparison with propagated communities originating from other original Mabisi samples the changes were parallel in direction. Our results showed that due to the new environment, species composition in communities changed. After 16 repeated propagation cycles two clusters, potentially representing two ecosystems, emerged among our propagated communities (Figure 5.2). The two ecological clusters were characterized by the fact that either Lactobacillus delbrueckii or Lactobacillus helveticus was the most abundant species. This implies these two species share the same ecological niche and seem to be interchanging their functionality. Interestingly, both species remained present in the population; the least abundant species was not completely outcompeted. They might have an additional role, or we are not at the endpoint of changes in species abundance yet. The differences we observed could 
potentially also be different stages of the same transition from the ancestor towards a most optimal species composition. Our results suggest that the change in species composition towards one of the two ecological states is dependent on the original Mabisi bacterial community. Predicting which cluster the species composition would belong to after repeated propagation cycles does not seem straightforward. For example, original Mabisi 4 and original Mabisi 5 show clear similarities (Figure 5.2 and Supplementary Figure 5.S1), while the communities after 16 propagation cycles belong to different clusters.

This dependence on the original bacterial community was apparent from our tests on the species composition model. The scenario that allowed for differences in initial species composition showed the biggest increase in likelihood. The environment was found to have the least influence on the change in species composition of the four tested mechanisms. It is interesting to see that allowing interactions between initial species composition and time explained an extra $18.6 \%$ of the variation, compared to the additive effect of initial species composition and time. This indicates that in one species composition a certain OTU increased, while in another species composition the same OTU decreased. We therefore hypothesise that biotic interactions within the community have a bigger influence on the fitness of a certain OTU than the selection of the abiotic environment.

One could hypothesize that the differences in the microbial community composition of all Mabisi translate into differences in metabolic activity. In contrast to the species composition, the metabolic profiles of the communities did not show two clusters representing the two ecological states. The metabolites produced in a microbial community might be more dependent on the environment than on initial and current species composition. The metabolic pathways resulting in the formation of the volatiles measured might be either present in species which are represented in both ecological states or be carried by different species but expressed in a similar way. Also, in $\mathrm{pH}$, phase separation and product thickness no directed change during the repeated propagation cycles were found (results not shown). We conclude that despite clear differences between the species composition after repeated propagation cycles, the metabolic functionality of the communities as a whole remained similar.

\section{Factors defining changes in species composition after repeated propagation cycles}

This experiment can also be seen as an analogy to an experimental evolution with one species starting with standing variation $(91,92)$. In our case, however, we study the sorting of species rather than the sorting of genotypes. Due to selection pressures, one or a few individuals with the highest fitness can be selected in an experiment with standing genetic variation. In case of communities we cannot speak about fitness of the individual, as the community does not reproduce as one organism, but rather consists of multiple organisms which all reproduce on their own and therefore have their own fitness. However, although we cannot use fitness as defined for individual genotypes within a species, we can study how whole communities may adapt to a new environment. Although species are often studied in isolation, in nature they interact 
with many other organisms. Therefore, this study is focussed on the dynamics of whole communities to complement findings of studies focussing on individuals.

New environments can cause communities to change in species composition and function. These changes are influenced by adaptation, chance, and evolutionary history (169). Trait that are strongly related to fitness (such as bacterial growth rate) are more influenced by history, while traits that are weakly related to fitness (such as cell size) are more influenced by environment and chance. In this study we analysed two traits of a bacterial community: species composition and metabolic profile. Species composition was more influenced by the initial species composition, while for metabolic profile this was not the case. It might therefore be that species composition in itself is not important for community 'fitness' or success, where success is reached by the highest efficiency in utilisation of the resources by the community. The selection might be on the level of active genes, independent on the bacteria in which these genes are present. This can be tested in future research by analysing the RNA profile of the community and check whether that is more related to the bacterial composition or the aroma profile. Potentially there are fewer optimal solutions for resource use which can be achieved by variations in species composition. This makes that community metabolism and community composition are not directly linked.

\section{Limitations}

We assumed that lactic acid bacteria (LAB) are the most abundant species in the context of milk fermentation. Therefore, in analysing species composition we only analysed bacteria by sequencing DNA encoding 16S rRNA. As Mabisi is produced by spontaneous fermentation, the natural community used in this experiment very likely contained organisms from other taxa. The possible interactions between taxa, such as with yeasts, fungi, or bacteriophages, might indicate why in some communities Lactobacillus delbrueckii had the highest presence, while in other communities Lactobacillus helveticus is most abundant. Further sequencing using primers that capture this diversity of taxa can give insight in these hypothesis. Another way of sequencing, such as deep sequencing or sequencing using targeted primers, can also give more insight in the microorganisms present to a subspecies or perhaps even lineage level. As subspecies are substantially different in their biology and functionality, grouping bacteria by their species level is not detailed enough to determine their function in a certain environment.

\section{Conclusion}

When put in a new environment, different natural microbial communities from Mabisi keep their diversity and did not show simple convergent change towards a microbial community with only a few species. Even though the species composition from the original Mabisi communities seem very similar, small differences made that the final species composition differed between samples from different origin. These changes were parallel in all 5 replicates of the same original Mabisi community. Initial composition and interactions were determining factors in species composition. However, final species composition could not be predicted by initial bacterial species composition. Despite the changes in species composition, a directed change in function 
was not found. Indicating that different groups of bacteria might have the same function in this system.

In summary, we saw that bacterial species composition was highly dependent on initial species composition and to a lesser extent depended on changes caused by the new environment. The reproducibility in the way these communities changed in their new environment opens a door for further research towards finding specific causes within the initial community for the specific compositional dynamics we observed. This would be an important step towards predicting community structure and function in novel environments.

\section{Supplementary material}

Supplement Table 5.S1 Shannon index as measure for bacterial diversity on OTU level of all six Mabisi samples, at the beginning of the transfers (T0) and the average after 16 transfers (T16).

$\begin{array}{lll} & \text { T0 } & \text { T16 (average) } \\ \mathbf{1} & 1.92 & 1.48 \\ \mathbf{2} & 2.00 & 1.84 \\ \mathbf{3} & 2.11 & 1.86 \\ \mathbf{4} & 1.75 & 1.45 \\ \mathbf{5} & 1.85 & 1.95 \\ \mathbf{6} & 2.03 & 1.75\end{array}$



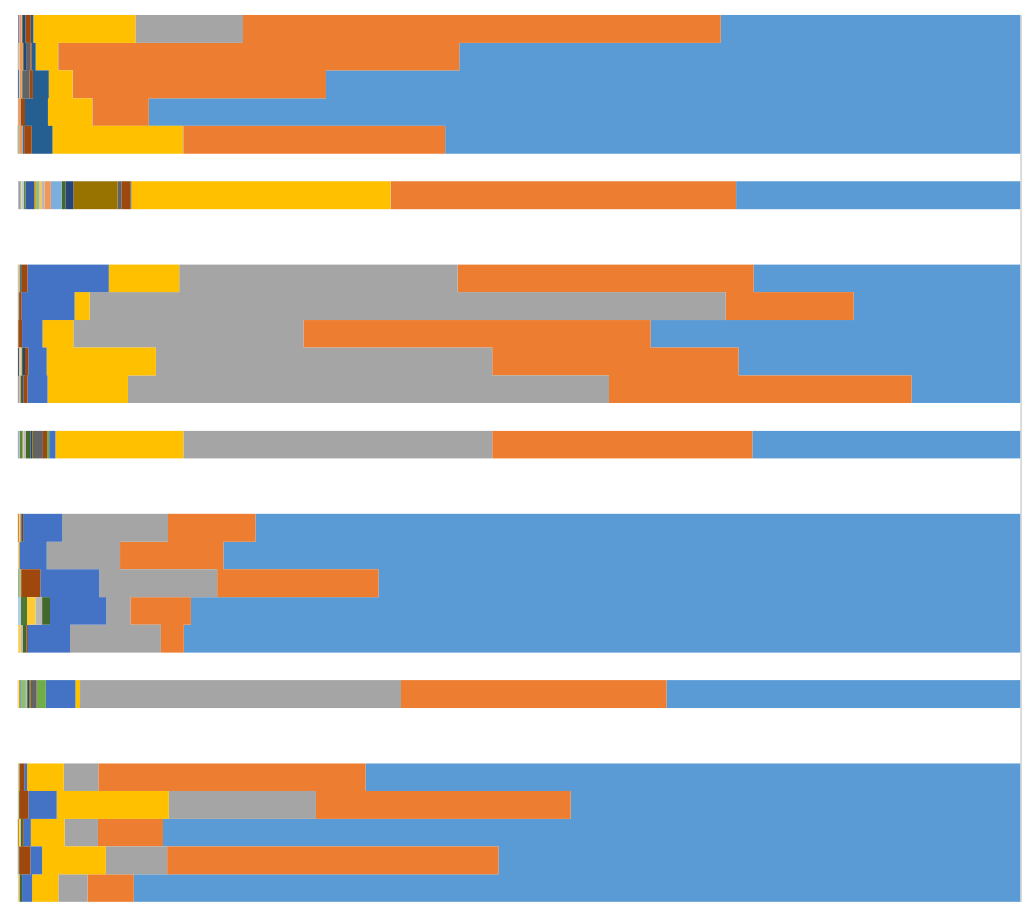

TO 4

T16 3E

T16 3D

T16 3C

T16 3B

T16 3A

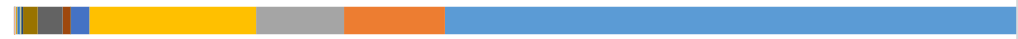

TO 3
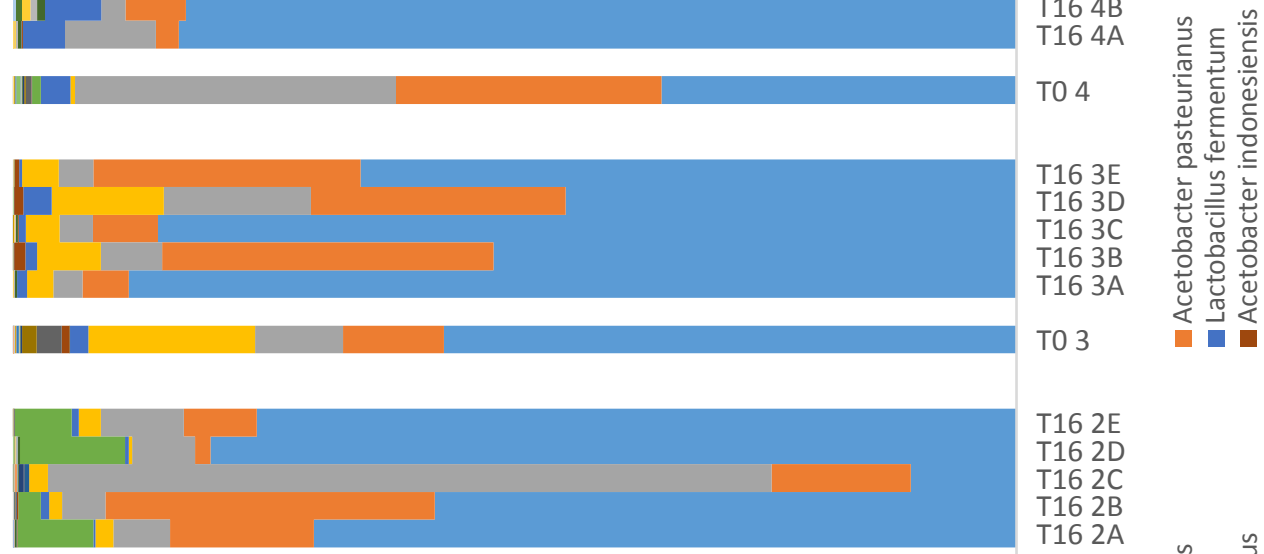

T16 2E

T16 2D

T16 2C

T16 2B

T16 2A

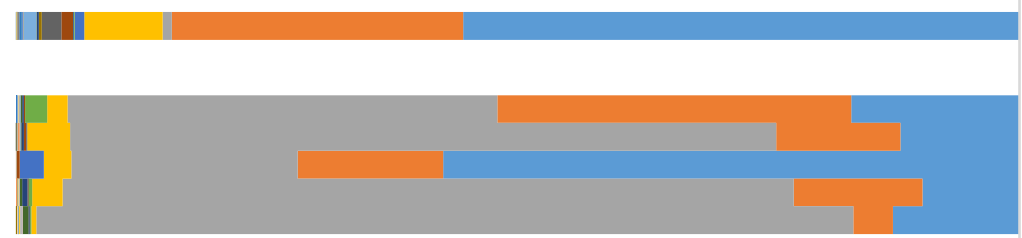

TO 2

T16 1E

T16 1D

T16 1C

T16 1B

T16 1A

||

TO 1

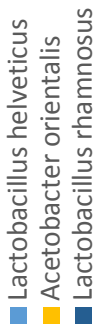

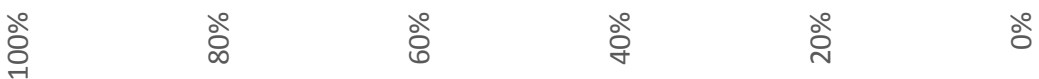

Supplement Figure 5.S1 Community structure on species level of all Mabisi samples at time point 0 and 16. Different colours indicate different species which could consist of multiple OTUs. Vertical axes indicate percentage of total reads within the sample. The nine species with highest abundance are indicated in the legend. The aim of the figure is to show the diversity found in the microbial communities of Mabisi in terms of species variation. For specific abundances of species and OTU, please refer to the sequencing data provided with this publication. 


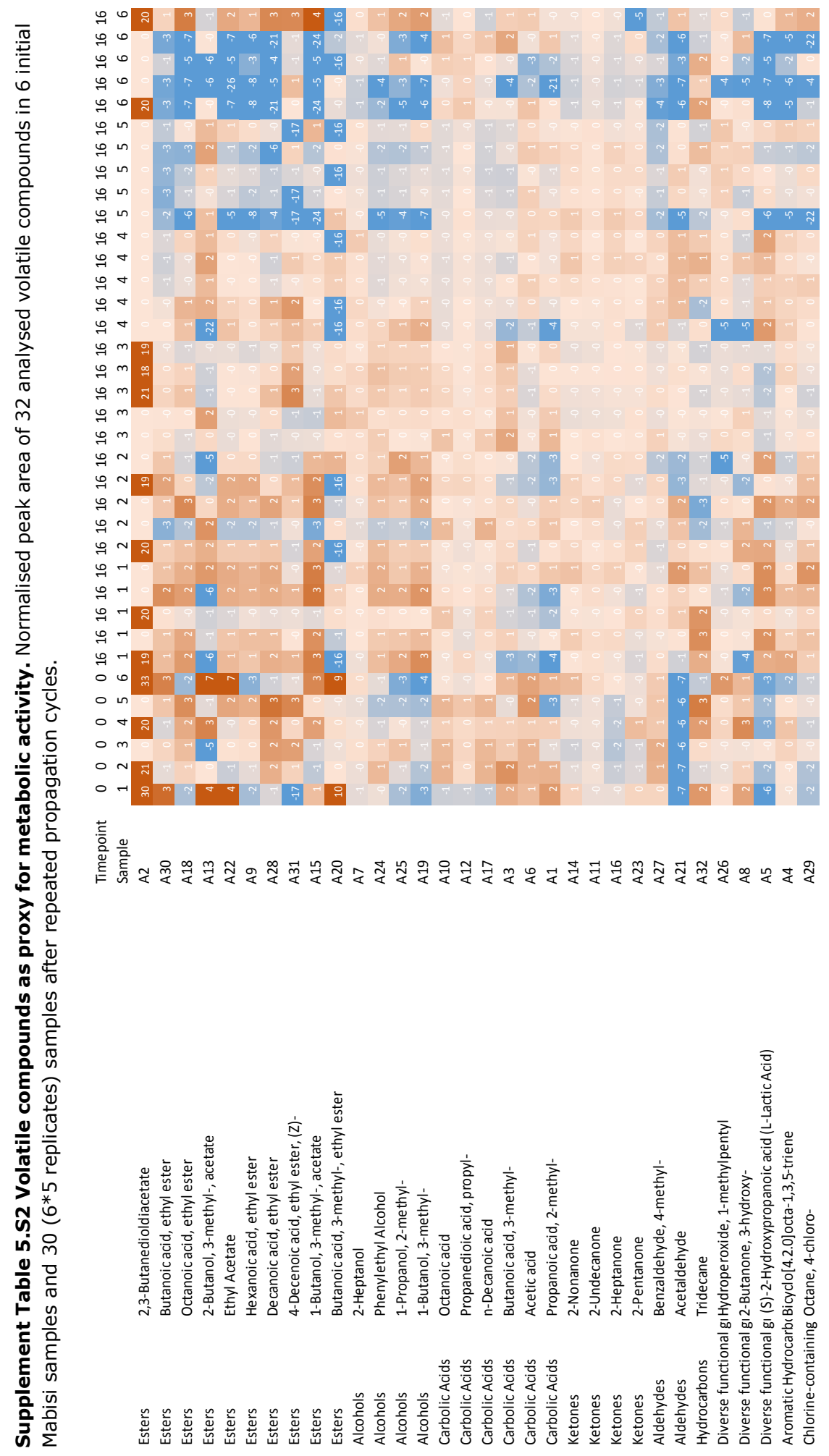



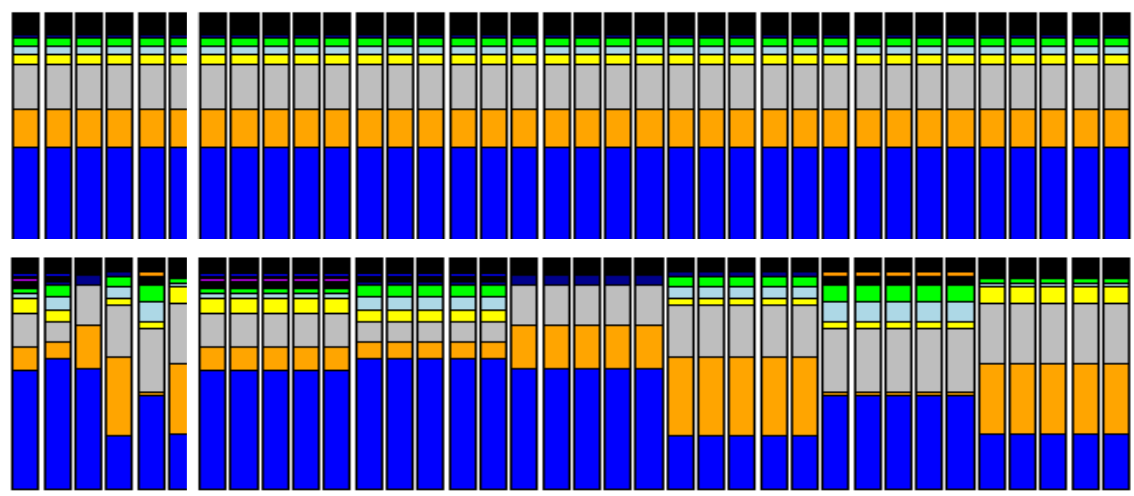

S1
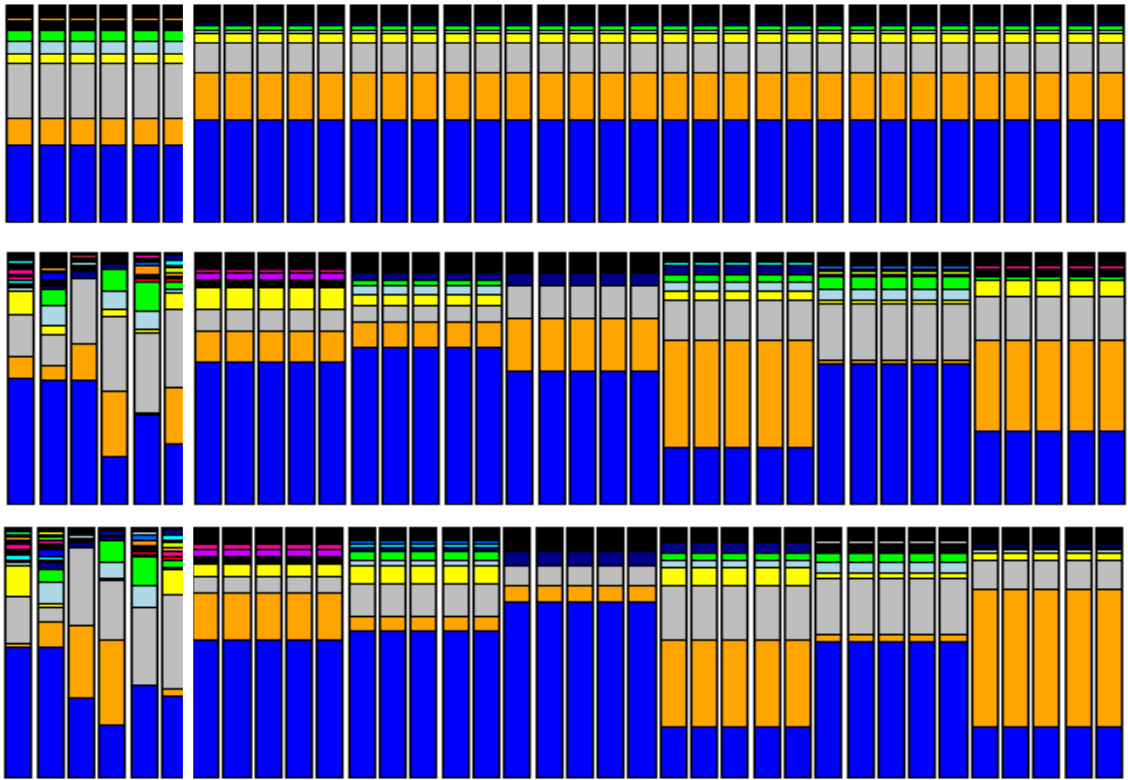

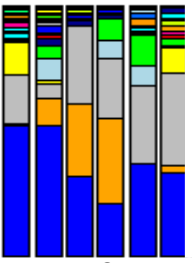

TO
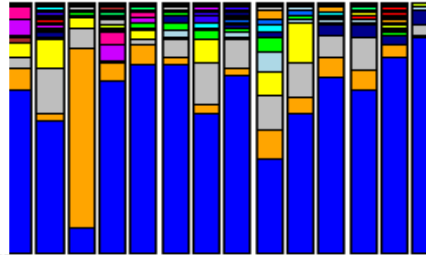

T16

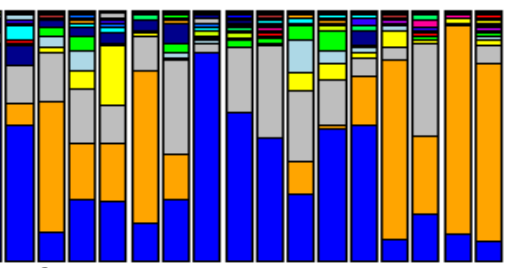

Supplement Figure 5.S2 Hypothetical community structures when allowing various factors in the construction of the communities from the total OTU pool. S0, all samples equal, no initial differences no frequency changes; S1, "Initial species composition", differences between sample, no changes in frequency; S2, "Time", similar frequency changes, no differences initially; S3, "Initial species composition + Time", equal frequency changes and initial differences; S4, "Initial species composition * Time", different frequency changes and initial differences; S5, "Initial species composition * Time + Stochasticity", all samples at T16 a different distribution. 


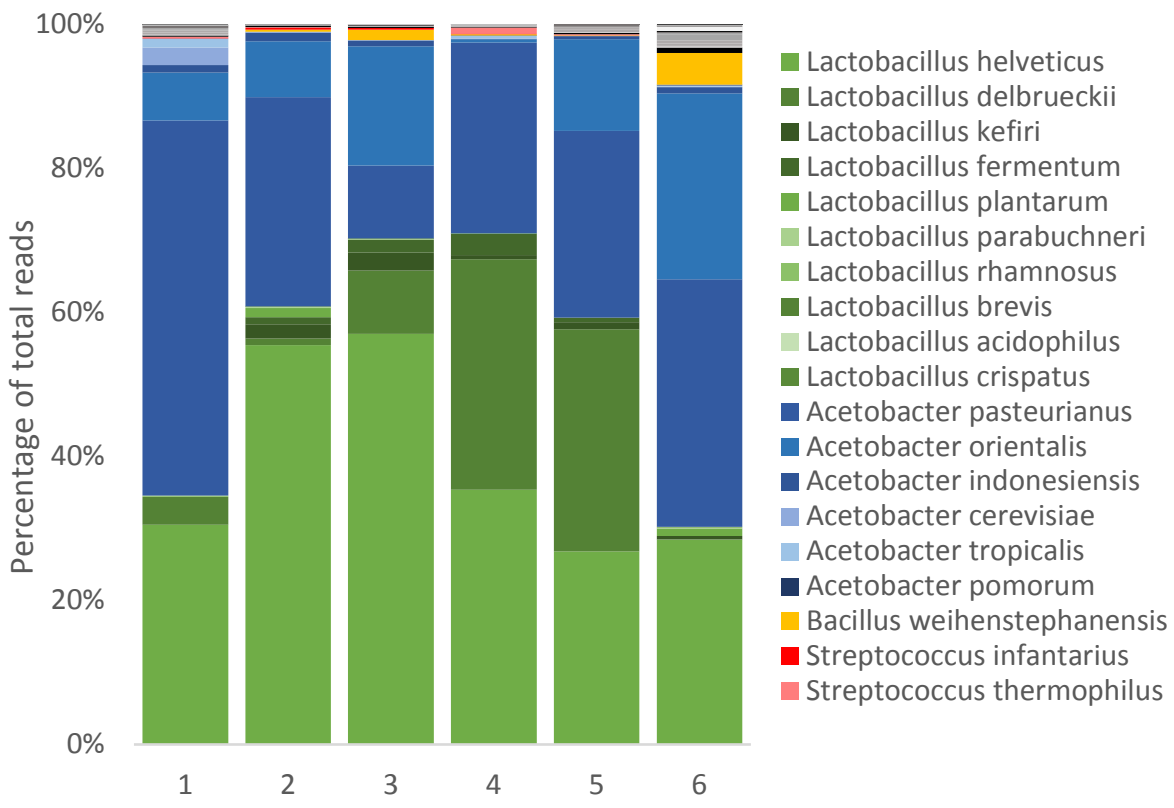

Supplement Figure 5.S3 Initial composition of natural communities from Mabisi. The aim of the figure is to show the diversity found in the microbial communities of Mabisi in terms of genus and species variation. Colours differentiate between the four most abundant genera: Lactobacillus (green), Acetobacter (blue), Bacillus (yellow) and Streptococcus (red). Shades of the colour indicate different species within that genera, which can consist of different OTUs. Shades of grey represent different species of other genera. 
Chapter 6. 


\title{
Bacterial community dynamics in Lait caillé, a traditional product of spontaneous fermentation from Senegal.
}

\author{
Anneloes E Groenenboom a,b \\ Megan E Parker ${ }^{c}$ \\ Anne de Vries ${ }^{c}$ \\ Suzette de Groot a \\ Stephanie Zobrist ${ }^{c}$ \\ Kimberly Mansen c \\ Peiman Milani ${ }^{c}$ \\ Remco Kort d,e,f,g \\ Eddy J Smid b \\ Sijmen E Schoustra ${ }^{a, h}$
}

Published in PloS ONE (2019)

\footnotetext{
a Laboratory of Genetics, Wageningen University and Research, Wageningen, The Netherlands

${ }^{b}$ Laboratory of Food Microbiology, Wageningen University and Research, Wageningen, The Netherlands

c PATH, Seattle, Washington, United States of America

d Microbiology and Systems Biology, TNO, Amsterdam, The Netherlands

e Yoba for Life Foundation, Amsterdam, The Netherlands

f Department of Molecular Cell Biology, VU University Amsterdam, Amsterdam, The Netherlands

${ }^{g}$ ARTIS-Micropia, Amsterdam, The Netherlands

h Department of Food Science and Nutrition, University of Zambia, Lusaka, Zambia
} 


\section{Abstract}

Spontaneously fermented food products contain a complex, natural microbial community with potential probiotic activity. The addition of a health-promoting, probiotic bacterium to these products ensures the delivery of that probiotic activity to consumers. Here, we assess the microbial community of a traditional Senegalese milk product produced by spontaneous fermentation, called Lait caillé. We produced the Lait caillé in a traditional way and added a probiotic starter containing Lactobacillus rhamnosus yoba 2012 to the traditional process. We found various species that are known for their ability to ferment milk, including species from the genera Lactobacillus, Acetobacter, Lactococcus, and Streptococcus. Our results show that the addition of $L$. rhamnosus to the inoculum, can result in detectable levels of this strain in the final product, ranging between 0.2 and 1 percent of the total bacterial population. Subsequent rounds of fermentation using passive back-slopping without the addition of new L. rhamnosus led to a loss of this strain from the community of fermenting bacteria. Our results suggest that the addition of probiotic strains at every fermentation cycle can enrich the existing complex communities of traditionally fermented Lait caillé while traditional bacterial strains remain dominant in the bacterial communities.

\section{Introduction}

Fermented foods are consumed worldwide and are of increasing interest to the field of public health. They include (complex) microbial communities of mainly lactic acid bacteria that are considered beneficial in stimulating a healthy gut microbiota and that promote food safety $(120,170)$. Most traditional fermented foods are produced by spontaneous fermentation, meaning that no starter culture is added to ferment the raw ingredients. Spontaneous fermentation results in products containing diverse microbial communities. Bacterial strains in these communities can be similar to probiotics (9). The probiotic properties of these products may be further enhanced by enriching the microbial communities through the addition of known probiotic strains to ensure probiotic activity.

In this study, we characterized the microbial community of Lait caillé, a fermented milk product from Senegal (171-174) using repeated rounds of traditional preparation as well as preparations including a probiotic starter culture. Lait caillé ("curdled milk") is traditionally produced by the spontaneous fermentation of cow's milk. The milk is first boiled and subsequently cooled before it is poured into a lahal-a wooden bowl that serves as the container for fermentation. Because lahals are used in succession, they contain a biofilm layer with the microbial culture from the previous fermentation cycle which then starts fermentation of the next batch.

First, we analyzed the composition and batch-to-batch dynamics of the microbial community in Lait caillé. The microbial community in other spontaneously fermented milk consist of various lactic acid bacteria (LAB) in combination with other bacteria, as well as yeasts and molds $(5,81-84,139,175,176)$. In the current study we focused on the bacterial composition of the final product in relation to the composition of biofilms in the lahals and the dynamics over multiple rounds of fermentation. Second, we studied the possibility of enhancing the probiotic properties of the final product, through addition of a probiotic starter culture to the initial microbial community. This 
starter culture, called "Yoba for Life", was developed for the production of probiotic yogurt (120) and consists of a mixture of two bacterial strains, Lactobacillus rhamnosus yoba 2012 and Streptococcus thermophilus C106. We applied the Yoba starter culture at the start of fermentation, into lahals containing their own natural, complex microbial communities and tracked the presence of the two added strains. Additionally, in some lahals we added millet porridge, as an extra fermentable substrate for the invading probiotic bacteria, which might give them an advantage over the existing fermenting community. This laboratory-based research is complementary to the fieldwork of Parker and colleagues (172), who analyzed microbial communities of Lait caillé produced in Senegal. The aim of this research was to increase the availability and accessibility of health-promoting fermented foods in regions with a high prevalence of undernutrition. Furthermore, we intended to contribute to the general knowledge on bacterial community stability, and the factors influencing bacterial invasion in existing microbial communities.

\section{Materials and methods}

\section{Bacterial strains and growth media}

We used bacterial invaders from the Yoba for Life starter culture for probiotic yogurt (CSK food enrichment, Leeuwarden, The Netherlands). This starter culture consists of Lactobacillus rhamnosus yoba 2012 and Streptococcus thermophilus C106 (120). We used sachets containing one gram of dried starter culture, containing at least $1 * 10^{\wedge} 9$ $\mathrm{CFU} / \mathrm{g}$ of each strain (177), to inoculate $1 \mathrm{~L}$ Ultra-high temperature (UHT) processed milk (Milbona, Lidl). MRS (Sigma-Aldrich, St. Louis, Missouri, United States) agar plates were incubated anaerobically at $28^{\circ} \mathrm{C}$ for the CFU count of L. rhamnosus. M17 (Fluka, Sigma-Aldrich) agar plates were incubated anaerobically at $42{ }^{\circ} \mathrm{C}$ for the CFU count of the thermophile $S$. thermophilus.

\section{Fermentation in the lahals}

Eight lahals were imported from different regions in Senegal (locations are reported in Supplement Table 6.S2) and their biofilm was sampled upon arrival in the laboratory (Supplementary Table 6.S1). From every lahal, $4 \mathrm{~cm}^{2}$ of the inside of each lahal was superficially scraped using a spatula. The obtained wood sample containing the microbial biofilm (inoculum for the fermentations) in these lahals was suspended in 1 $\mathrm{mL}$ of saline $(0.8 \%$ (wt/vol) $\mathrm{NaCl}$ solution). The lahals were filled with $1 \mathrm{~L} \mathrm{UHT}$ milk (Milbona, Lidl) and incubated at $28{ }^{\circ} \mathrm{C}$ for 3 days to allow the first round of fermentation. Samples $(1 \mathrm{~mL})$ of the resulting fermented milk were stored at $-20{ }^{\circ} \mathrm{C}$ and in glycerol $(0.5 \mathrm{~mL})$ at $-80^{\circ} \mathrm{C}$. After use, the lahals were cleaned with sterile water and tissue paper. Immediately after the cleaning, a biofilm samples was taken from the inside of the lahals. The lahals were used for fermentations and regularly sampled for 4 months. MRS (Sigma-Aldrich, St. Louis, Missouri, United States) agar plates were incubated anaerobically at $28^{\circ} \mathrm{C}$ for the total count of lactic acid bacteria as measure for total bacterial count.

\section{Standard Yoba yogurt}

A standard Yoba yogurt was produced by adding $1 \mathrm{~g}$ Yoba for Life starter culture to 1 L UHT milk in a sterile glass bottle. This bottle was incubated at $28^{\circ} \mathrm{C}$ overnight to allow fermentation. At different time points during fermentation samples were 
incubated anaerobically on M17 agar at $42{ }^{\circ} \mathrm{C}$ and MRS agar at $28{ }^{\circ} \mathrm{C}$ to count respectively $S$. thermophilus and $L$. rhamnosus. Resulting counts were used to calculate average growth rates in standard Yoba yogurt. The DNA was extracted from the resulting yogurt for analyses of $L$. rhamnosus and $S$. thermophilus concentrations, which were used as the standard for probiotic characteristics.

\section{Probiotic invasion}

In the eight lahals, the following propagation environments were tested: 1 ) the addition of $1 \mathrm{~g}$ Yoba starter culture to $1 \mathrm{~L}$ UHT milk at fermentation cycle 1 and cycle 7 (for three lahals); 2) the addition of $1 \mathrm{~g}$ Yoba culture starter to $1 \mathrm{~L} \mathrm{UHT} \mathrm{milk} \mathrm{at} \mathrm{fermentation}$ cycle 1 and cycle 7 and a one portion of millet porridge at all twelve fermentation cycles (for three lahals); and 3) controls consisting of only 1 L UHT milk (two lahals). To produce one portion of millet porridge, $50 \mathrm{~g}$ millet flour was mixed with $400 \mathrm{~mL}$ water and heated in a microwave (750W). Heating was alternated with stirring every 30 seconds, until the porridge was boiling (typically after 4.5 minutes). The porridge was left to cool at room temperature for 30 minutes. Lahals were covered with aluminum foil and incubated at $28{ }^{\circ} \mathrm{C}$ for two days and used for fermentation twelve times consecutively. Community dynamics were analyzed with non-culture based sequencing methods targeting the $16 \mathrm{~S}$ rRNA region of the bacterial DNA.

\section{DNA extraction}

The DNA extraction method was adapted from Ercolini et al. 2001 and Schoustra et al. 2013 (5,140). For DNA extraction, $1 \mathrm{~mL}$ of fermented milk was spun down (2 minutes, 12000 RPM), after which the supernatant was removed. The cells were re-suspended in a mix of $64 \mu \mathrm{L}$ EDTA $(0.5 \mathrm{M}), 160 \mu \mathrm{L}$ Nucleic Lysis Solution, $5 \mu \mathrm{L}$ RNAse, $120 \mu \mathrm{L}$ lysozyme and $40 \mu \mathrm{L}$ pronase $\mathrm{E}$. After an incubation time of 60 minutes at $37^{\circ} \mathrm{C}$ and agitation of $350 \mathrm{RPM}, 400 \mu \mathrm{L}$ ice-cold ammonium acetate (5 M) was added and the mixture was cooled on ice for 15 minutes. The mixture was spun down and $750 \mu \mathrm{L}$ of supernatant was transferred to a tube containing $750 \mu \mathrm{L}$ phenol. This tube was vortexed and its content spun down ( 2 minutes, $12000 \mathrm{RPM}$ ) and $500 \mu \mathrm{L}$ of supernatant was transferred to a tube containing $500 \mu \mathrm{L}$ chloroform. This tube was vortexed and its content spun down ( 2 minutes, 12000 RPM) and $400 \mu \mathrm{L}$ of supernatant was transferred to a tube containing $1 \mathrm{ml} 100 \%$ ethanol and $40 \mu \mathrm{L}$ sodium acetate $(3 \mathrm{M})$. This tube containing DNA was left to precipitate at $-20^{\circ} \mathrm{C}$ overnight. The next day, the tube was spun for 20 minutes at 12000 RPM at $4{ }^{\circ} \mathrm{C}$. The supernatant was carefully aspirated and the DNA pellet was washed by adding $1 \mathrm{~mL} 70 \%$ ethanol. The tube was spun for 10 minutes at $12000 \mathrm{RPM}$ at $4^{\circ} \mathrm{C}$, after which the supernatant was aspirated again. The DNA pellet was left to dry at room temperature and dissolved in $20 \mu \mathrm{L} 10$ $\mathrm{mM}$ Tris $\mathrm{pH}$ 7.5.

\section{DNA sequencing and sequence analyses}

The extract containing DNA from all organisms in the community was sent for pairedend sequencing of the $\mathrm{V} 4$ hypervariable region of the bacterial 16S rRNA gene amplicon (341F-785R) on the MiSeq Illumina platform by LGC genomics (Berlin, Germany).

For further data processing and statistics the QIIME pipeline (141), modified from Bik et al. (142) was used. Paired-end reads were joined using join_paired_ends.py (with 
minimum overlap 10 basepairs) after which sequences were trimmed and filtered using cutadapt (v1.11 -q 20, -m 400 (154)) using the known primer sequences CCTACGGGNGGCWGCAG and GACTACHVGGGTATCTAAKCC to trim both sides of the sequence. These trimmed sequences were then checked for chimera's, using uchime (v4.2.20, gold database (155)). Sequences with a chimera score lower than 0.28 were retained. Next, sequences were clustered into operational taxonomic units (OTUs) after quality check using pick_open_reference_otus.py (-s 0.1 , -enable_rev_strand_match TRUE, -align_seqs_min_length 75, -pick_OTU_similatiry 0.95). Taxonomy of the resulting OTUs was assigned to representative sequences using the Greengenes (v13.5) rRNA database. This algorithm gives a representative sequence for an OTU, which were used to perform a local BLAST using the gold database from uchime (146). The outcome of the top BLAST hit was used for further data processing. Shannon index (H) accounts for both abundance and evenness of OTUs present and is calculated using $\mathrm{H}=-\Sigma \mathrm{pi}$ In $\mathrm{pi}$ in which $\mathrm{pi}$ is the proportion of species i relative to the total number of species(178).

\section{Results and discussion}

\section{Bacterial composition of Lait caillé}

\section{Initial composition of microbial communities in lahals}

Upon arrival of the lahals in the laboratory, we analyzed the bacterial composition of their internal surface. Because the lahals are wooden, they exhibit a rough internal surface that facilitates the formation of biofilms and the accumulation of residues from in each fermentation cycle. Species of Lactobacillus and Streptococcus are the most abundant across all community structures found on the lahals' surfaces; $56.8 \%$ and $16.8 \%$ of the total reads respectively (Figure 6.1 ). However, substantial variation between lahals was found both in terms of types of species present as well as overall species diversity. The Shannon index $(\mathrm{H})$ is a measure of diversity based on operational taxonomic units (OTUs). The Shannon index varied from 1.98 to 3.20 and can be found per lahal in Supplement Table 4.S1. The community structures we find here are in line with the findings of Parker et al. (172), who analyzed the same type of lahals in a field study in Senegal. We found various species that are known for their ability to ferment milk, such as Lactobacillus helveticus $(179,180)$ and Streptococcus thermophilus $(181,182)$. Not all species are likely to contribute to the milk fermentation. We found species that are known for their presence in the environment and animals, including Pseudomonas libanensis (183) and Buttiauxella warmboldiae (184), which have a high presence in lahal 1 (18.9\% and $10.6 \%$, respectively), and Kurthia gibsonii (185), which has a high presence in lahal 2 and 4 (33.9\% and $11.6 \%$ respectively). Presence of species like Lactobacillus delbrueckii confirmed that the communities in the lahals can contain probiotic strains $(186,187)$. However, with the analyses used in this study, the identification of the bacteria at the level of subspecies or lineage is not possible and therefore no conclusions regarding the presence of probiotic strains can be made.

The spontaneous nature of the fermentation explains our finding that the seven lahals contain microbial communities of varying compositions. A cluster analysis did not reveal a relationship between the community structure and the origin, frequency of use, or age of the lahals (Supplement Table 6.S2). However, the lahals were used by different producers, who might have different methods, which may have influenced the 
influx of various bacteria as well as the selection pressure on the various bacterial species.

\section{Similarity between lahal biofilm community and microbial composition in the resulting Lait caillé}

Spontaneous fermentation of Lait caillé differs slightly from the process of active backslopping, where a sample from a previous fermentation round is used to start a new fermentation $(13,188)$. We therefore refer to this process as "passive back-slopping", because an external starter culture is not intentionally added. The outcome of the first cycle of spontaneous fermentation is difficult to predict, as it is highly influenced by chance. Over time, through back-slopping, the fermenting community can stabilize and generate stable product characteristics $(77,79)$. In some traditional fermented dairy products, such as Parmigiano Reggiano (12), the back-slopped community must compete with a microbial community present in the raw milk. For Lait caille this is not the case, as the raw milk is boiled before fermentation. We hypothesize that the communities in the lahals are the inoculum for the milk. Thus, the microbial community in Lait caillé will be a modified subset of the bacterial community in the lahals' biofilm.

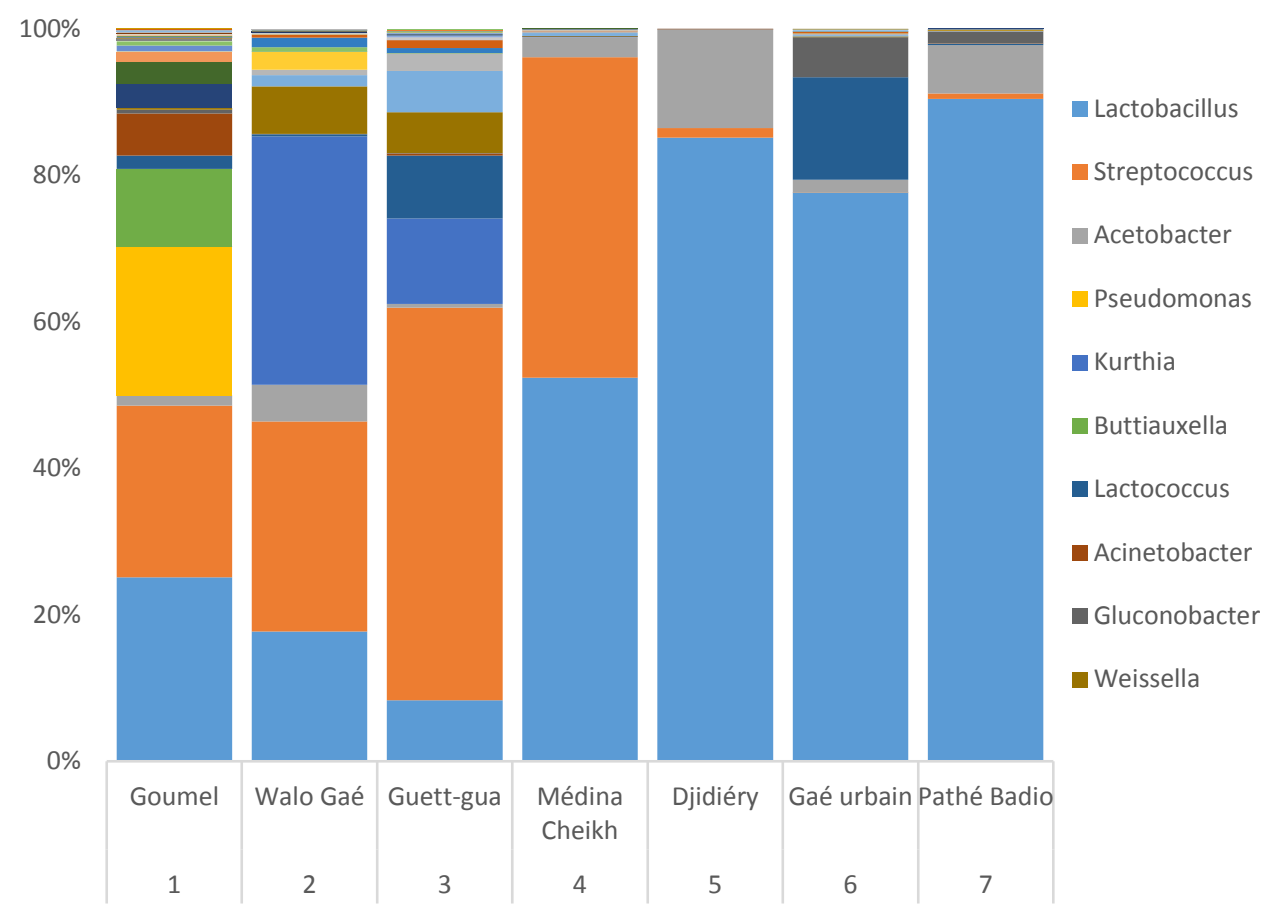

Figure 6.1 Bacterial composition of biofilm samples of all lahals upon arrival at the laboratory. Different colors indicate different genera and can consist of different species and different OTUs. The ten genera with highest abundance are indicated in the legend. The aim of the figure is to show the diversity found in the microbial communities of Lait caille in terms of genus and species variation. For specific abundances of species and OTU, please refer to the sequencing data provided with this publication. Lahal numbers are used consistently throughout the experiments. Locations indicate the origin of the lahals. 
Upon arrival at our laboratory, the lahals were used for several rounds of fermentation. Each round was initiated by adding $1 \mathrm{~L}$ of sterilized whole milk to each lahal, which was subsequently incubated at $28^{\circ} \mathrm{C}$ for 48 hours. We compared the microbial composition of Lait caillé (the final product) after one fermentation cycle with the microbial composition of the biofilm in the lahal. Figure 6.2 shows a cluster analysis of the bacterial biofilm and final product communities of lahals 2, 3, 4, 6, and 7 . This shows that biofilm samples and Lait caillé samples cluster more often together than each Lait caille sample clustering with the corresponding biofilm sample. Considering that the biofilm is the only inoculum in the sterilized milk, we might expect some more overlap. However, multiple strains present in the biofilm do not grow in milk. The most abundant OTUs were different for every Lait caillé sample, but for every individual Lait caillé, the three most common OTUs in could be traced into the corresponding lahal biofilm samples. However, some OTUs from Lait caillé are not detected in the biofilm samples. It is plausible that these bacteria were present in the biofilm at a level below the detection limit and have the chance to grow significantly when milk is added to the lahal (3).

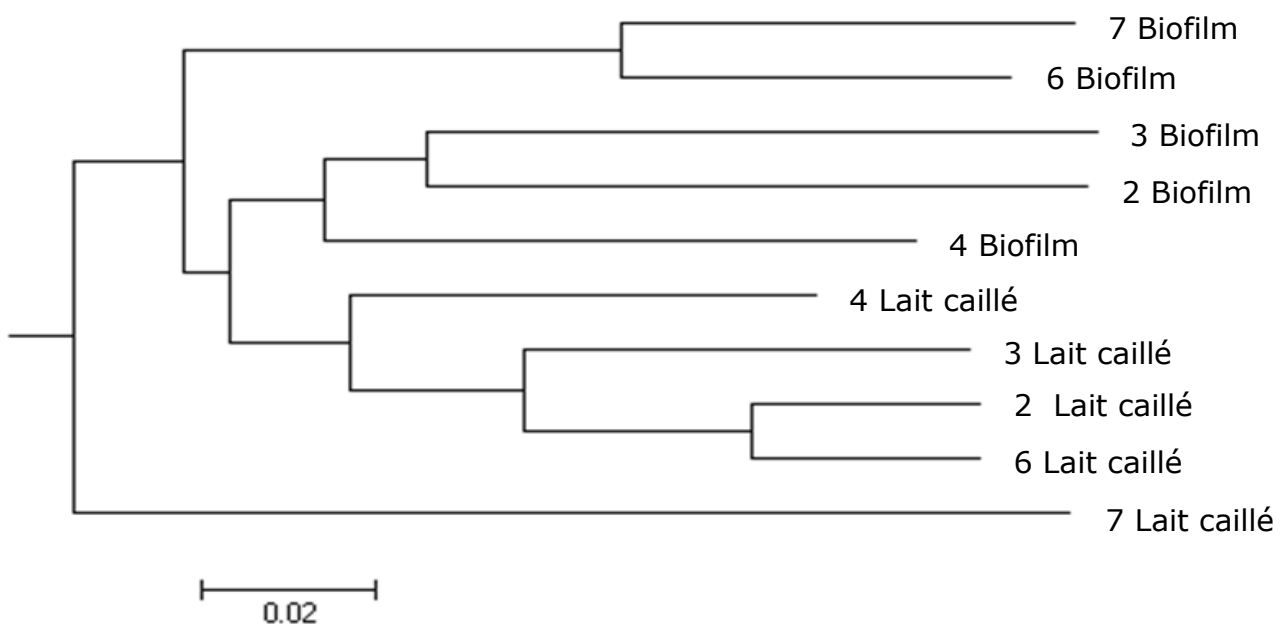

Figure 6.2 Hierarchical clustering of bacterial communities from lahal surface and Lait caillé. Lahal surface samples as well as Lait caillé samples of lahal 2,3,4,6, and 7 are clustered using Unweighted Pair Group Method with Arithmetic Mean based on beta-diversity of the bacterial communities.

\section{Changes in the microbial composition of Lait caillé over multiple rounds of fermentation}

To assess the temporal stability of the bacterial communities in the final products of three lahals, a selection experiment was conducted. These lahals were used in Senegal to propagate the communities over many cycles, allowing them to adapt to specific growth conditions. We may assume that the bacterial communities are in dynamic balance in terms of their species composition. Then, not taking erratic passersby into consideration, the bacterial frequencies will not change as much between fermentation cycles, provided that the incubation conditions in the laboratory are not very different from those in Senegal. The results (Figure 6.3) show that the communities of Lait caillé 
produced in lahals 2, 4, and 7 maintain a high diversity throughout the fermentation cycles (25 transfers in case of lahal 2). Over time, we see a change in the bacteria present in the Lait caillé samples. Lait caillé from both lahal 2 and lahal 9 is mainly dominated by Lactobacillus helveticus, Acetobacter pasteurianus, and Lactococcus lactis. Lait caillé from lahal 4 is mainly dominated by Streptococcus thermophilus, with Lactobacillus delbrueckii as the main Lactobacillus species after the first fermentation. After six fermentation rounds, Lactobacillus helveticus has the highest presence, together with Acetobacter pasteurianus and Lactococcus lactis. As this is also the main species present in Lait caillé from lahals 2 and 7, these strains might form a stable community. When comparing these data to the results reported by Parker et al. (172), we see that the percentage of Acetobacter species is higher, while that of Streptococcus species is lower in these Lait caillé samples. This might be due to the difference in fermentation time; each fermentation cycle in our experiment was 48 hours, compared to 12-24 in Parker et al. (6). Streptococcus thermophilus is known for its proteolytic activity and is therefore able to grow from the start of a fermentation $(27,52)$. Acetobacter species grow through oxidation of the fermentation products from the rest of the community (189), and will have their growth at a later stage in the fermentation. During this stage, the relative abundance of Streptococcus species might decrease due to a relative decrease in growth.

The difference between this selection experiment and most laboratory selection experiments using bacterial cultures (experimental evolution), is the mode of bacterial transfer between fermentation cycles $(41,190-192)$. Rather than transferring the liquid phase of the final product the lahals themselves are "transferred" to the next round of fermentation, in the sense that each lahal contains a biofilm that acts as the starter culture. This mode of transfer represents passive back-slopping, that is part of traditional Lait caillé production methods. As a result, bacteria are under selection not only to grow fast in milk, but also attached to the lahals' inner surfaces, either by formation of a biofilm, or through residing in a deep crevice in the wood of the lahal.

\section{Community diversity}

The bacterial diversity of the communities did not decrease throughout the fermentation cycles in the laboratory (Figure 6.3), which suggests that niches in the system have all been occupied in a temporally stable way. This may limit the opportunity for other bacteria such as probiotic bacteria, but also pathogens, to invade the communities. High diversity can result in a stable functionality of the community due to a back-up function $(17,68,99)$, causing a lower number of unoccupied niches, for example due to unused nutrients (100). Under such circumstances, those niches are not available for any invader, which makes the whole system more likely to keep its functionality and not destabilise due to a non-cooperator (69). However, the exact relationship between diversity and stability is still under debate $(98,193)$. 


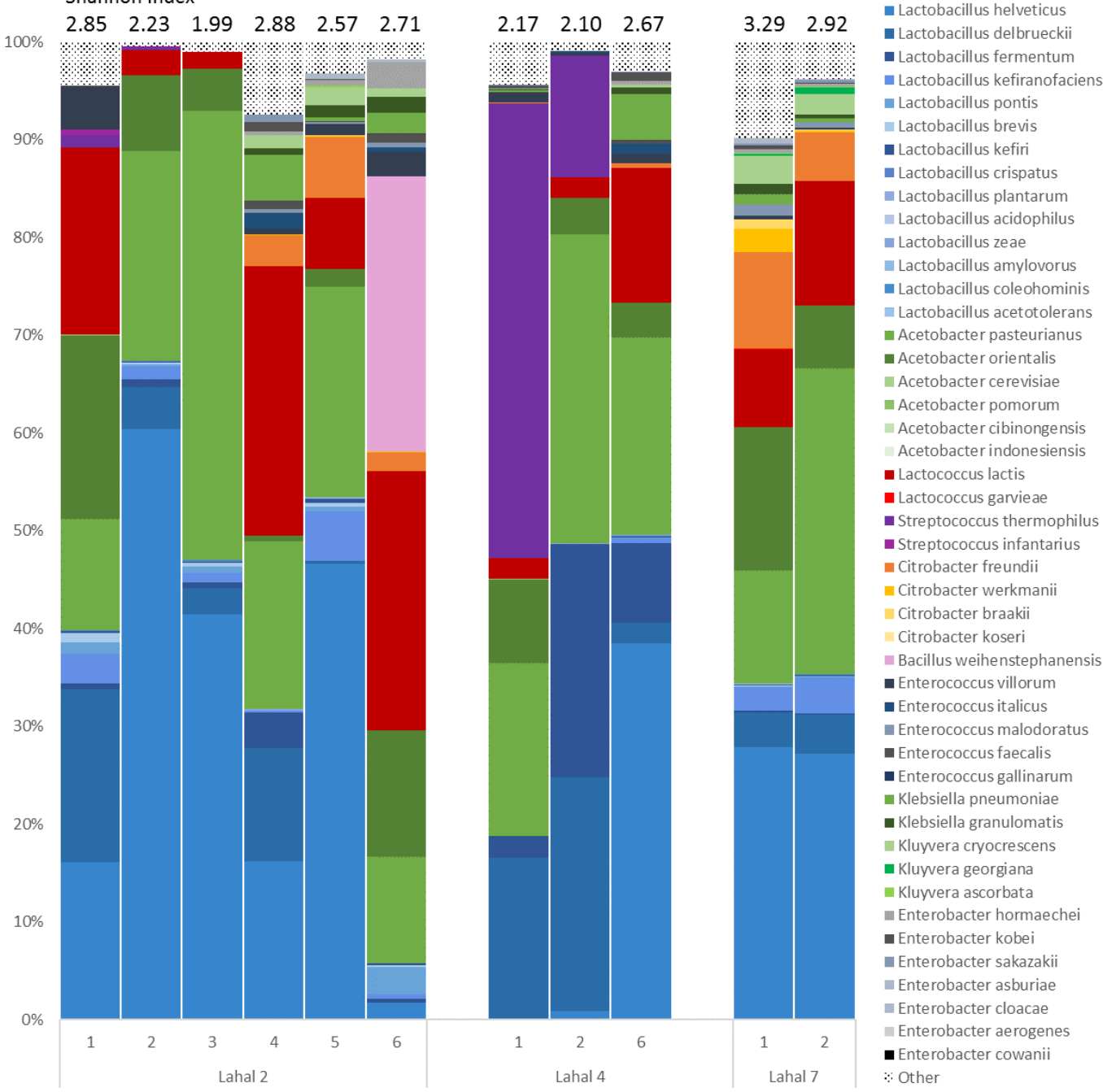

Figure 6.3 Community composition of bacterial species in Lait caillé of lahals 2, 4, and 7 over time. These lahals were used for fermentation by only adding sterile milk. Data points of lahal 4 and 7 are not shown as there were other ingredients added in these fermentation. Different colors indicate different species and can consist of multiple OTUs. Vertical axes indicates percentage of total reads within the sample. The aim of the figure is to show the diversity found in the microbial communities of Lait caillé in terms of genus and species variation. For specific abundances of species and OTU, please refer to the sequencing data provided with this publication.

\section{Addition of Yoba starter culture}

In the second part of this study, we attempted successful invasion by probiotic bacteria that were not present in the original community. If these probiotics were to become a permanent member of the bacterial community of a lahal, all products fermented in this lahal could have probiotic characteristics. 
Probiotics were added in a dried form; the Yoba for Life starter culture (120), containing Lactobacillus rhamnosus yoba and Streptococcus thermophilus. This starter culture is used in Uganda and other African countries to produce Yoba yogurt, a probiotic yogurt, and has been used before in studies on nutritional enrichment of traditional foods (194). The probiotic activity of Yoba yogurt and other foods that are enriched with the Yoba starter culture, is mainly attributed to L. rhamnosus, while $S$. thermophilus is necessary because of its proteolytic activity (177).

\section{Presence of $L$. rhamnosus and $S$. thermophilus in traditional Lait caillé}

Before adding a starter culture, we screened the bacterial communities of traditional Lait caillé for the presence of $L$. rhamnosus and $S$. thermophilus strains. The resolution of the 16S rRNA encoding DNA amplicon is too low to distinguish between wild $L$. rhamnosus strains and the $L$. rhamnosus yoba 2012 from the Yoba starter culture. The presence of OTUs representing $L$. rhamnosus and $S$. thermophilus in traditional Lait caille therefore has an influence on the trustworthiness of the findings in Lait caillé produced with the Yoba starter culture. All Lait caillé without Yoba starter culture tested negative for $L$. rhamnosus OTUs, while the samples from standard Yoba yoghurt and Yoba-enriched Lait caillé tested positive (Table 6.1). This implies that the OTUs that did blast as a L. rhamnosus indeed originated from the Yoba starter culture as this strain is below detection limits in traditional Lait caillé. S. thermophilus was found in some traditional Lait caillé, which needs to be taken into account when analyzing bacterial communities of enriched Lait caillé.

Table 6.1 Wild type strains of $L$. rhamnosus and $S$. thermophilus in Lait caillé. Percentages and total numbers of OTUs found in Lait caille before the addition of Yoba starter culture, standard Yoba yogurt, and Lactobacillus rhamnosus Yoba isolated from the standard Yoba yogurt that blasts as $L$. rhamnosus or S. thermophilus. Column A: average, column S: standard error *values based on one measurement.

\begin{tabular}{|c|c|c|c|c|c|}
\hline & & $\begin{array}{l}\% \\
\text { L. rhamnosus }\end{array}$ & $\begin{array}{l}\% \\
\text { S. thermophilus }\end{array}$ & $\begin{array}{l}\text { \# OTUs } \\
\text { L. rhamnosus }\end{array}$ & $\begin{array}{l}\text { \# OTUs } \\
\text { S. thermophilus }\end{array}$ \\
\hline \multirow{2}{*}{$\begin{array}{l}\text { Lait caillé from } \\
\text { Lahal } 3\end{array}$} & A & 0 & 0.23 & 0 & 1 \\
\hline & $\mathrm{S}$ & 0 & 0.43 & 0 & 1 \\
\hline $\begin{array}{l}\text { Lait caillé from } \\
\text { Lahal } 4\end{array}$ & * & 0 & 21.96 & 0 & 26 \\
\hline \multirow{2}{*}{$\begin{array}{l}\text { Lait caillé from } \\
\text { Lahal } 6\end{array}$} & A & 0 & 19.65 & 0 & 46 \\
\hline & $\mathrm{S}$ & 0 & 19.67 & 0 & 38 \\
\hline $\begin{array}{l}\text { Lait caillé from } \\
\text { Lahal } 8\end{array}$ & * & 0 & 0 & 0 & 0 \\
\hline \multirow{2}{*}{$\begin{array}{l}\text { Lait caillé from } \\
\text { Lahal } 9\end{array}$} & A & 0 & 0.03 & 0 & 1 \\
\hline & $\mathrm{S}$ & 0 & 0.03 & 0 & 1 \\
\hline $\begin{array}{l}\text { Standard Yoba } \\
\text { yogurt }\end{array}$ & * & 0.29 & 99.26 & 3 & 286 \\
\hline $\begin{array}{l}\text { Isolated L. } \\
\text { rhamnosus Yoba }\end{array}$ & * & 99.73 & 0 & 99 & 0 \\
\hline
\end{tabular}

\section{Yoba starter culture concentration after systematic addition of invaders}

As the next step, we performed an invasion experiment to assess if the concentration of Yoba bacteria in Lait caillé can become as high as in standard Yoba yogurt, in this 
way reaching levels for the product to be considered probiotic. Standard Yoba yogurt has a bacterial concentration of $10^{6}$ to $10^{7} \mathrm{CFU} / \mathrm{mL}$, and contains mostly $S$. thermophilus with approximately $0.3 \%$ L. rhamnosus. Because most of the bacterial communities found in the lahals are relatively diverse, invasions into these communities will likely be difficult. To mitigate this, we added the invading bacteria to the community in very high amounts, so the invaders initially outnumber the biofilminhabiting bacteria by a factor of 10 . We measured $6.36 \pm 2.47 \times 10^{5} \mathrm{CFU} / \mathrm{mL}$ bacteria released from the biofilm 10 minutes after the start of fermentation and $6.61 \pm 0.13 \mathrm{x}$ $10^{6} \mathrm{CFU} / \mathrm{mL}$ invading bacteria. A higher starting frequency of invaders improves their chances of integrating into the community before being outcompeted for nutrients, and increases their odds of outcompeting others by drift processes and chance.

After the addition of the Yoba starter culture at the start of the first and seventh fermentation cycle, we performed multiple rounds of fermentation in the same lahals without the addition of Yoba starter culture. In this way, we assessed the success of establishing the probiotic starter bacteria in the lahals' biofilms, which serves as the inoculum for all following Lait caillé fermentations in that lahal. The presence of invading strains was measured after completion of the fermentation cycle during which they were added (cycles 1 and 7), as well as some of the subsequent fermentation cycles (cycles 2 and $6 ; 8$ and 12, see Figure 6.4). The average percentage of $L$. rhamnosus in Yoba yogurt was used as a threshold baseline value to classify the Yobaenhanced Lait caillé as probiotic.

In fermentations where Yoba starter culture had been added, the two Yoba species made up between 4 and 80 percent of the total microbial population after 48 hours. In these fermentation cycles (cycles 1 and 7), L. rhamnosus reached concentrations similar to those found in standard Yoba yogurt (blue dashed line, Figure 6.4), while the concentrations of $S$. thermophilus stayed below the standard (orange dashed line). In the fermentation cycles that followed (cycles 2, 6, 8, and 12), the abundance of both L. rhamnosus and $S$. thermophilus had dropped to the level of the negative control lahals (black dotted line). Lahal 6 underwent 23 fermentation cycles. At this point, both L. rhamnosus and $S$. thermophilus concentrations were below the detection limit (indicated by * in Figure 6.4).

In order to provide the invading bacteria an advantage over the existing community in Lait caillé, another fermentable substrate, cooked millet porridge, was added at the beginning of every cycle in two lahals. Both L. rhamnosus and $S$. thermophilus are known to grow well when provided with millet porridge (195). The bacteria in the lahal are not adapted to millet as a substrate. When the community encounters a disturbance, this might open up the possibility for an invader to take a place in the community $(69,196)$. Addition of millet porridge is common practice in Lait caillé production in Senegal, although it is usually added prior to consumption rather than at the start of fermentation. In Figure 6.4, we see that the addition of millet porridge does not seem to affect the invasion by these bacteria. The concentrations either of the two species from the Yoba starter culture are comparable to the invasion without addition of millet porridge. Further research could investigate other nutrient sources which provide a more selective environment that benefits the added strains. 
In the field work of Parker and colleagues (172), the addition of Yoba starter culture to various lahals with Lait caillé resulted in a 20- to 60-fold increase in the total number of probiotic bacteria in the Lait caillé. These findings are in line with ours. Upon the addition of Yoba starter culture, we found a concentration of $L$. rhamnosus strains similar to the concentration found in standard Yoba yogurt. However, the invading strains were unable to successfully establish themselves in the lahals in order to classify the Lait caille produced in the following fermentation rounds as a probiotic product. L. rhamnosus was found in some biofilm samples, but in very low concentrations (results not shown). It is therefore unlikely that this strain established itself in the biofilm to provide a $L$. rhamnosus inoculum for the next batch of Lait caillé. When adding Yoba to a Zimbabwean milk product called mutandabota, Mpofu et al. (194) found that the concentration of $L$. rhamnosus yoba at the end of fermentation was $8.8 \mathrm{log} \mathrm{CFU} / \mathrm{ml}$, a considerably higher concentration than found in all samples of Lait caillé. An important difference between Lait caillé and mutandabota is that in the latter no original community is present with which the added strains would have to compete. Further research could include a selection step, to obtain probiotic bacteria that have the ability to form biofilms.

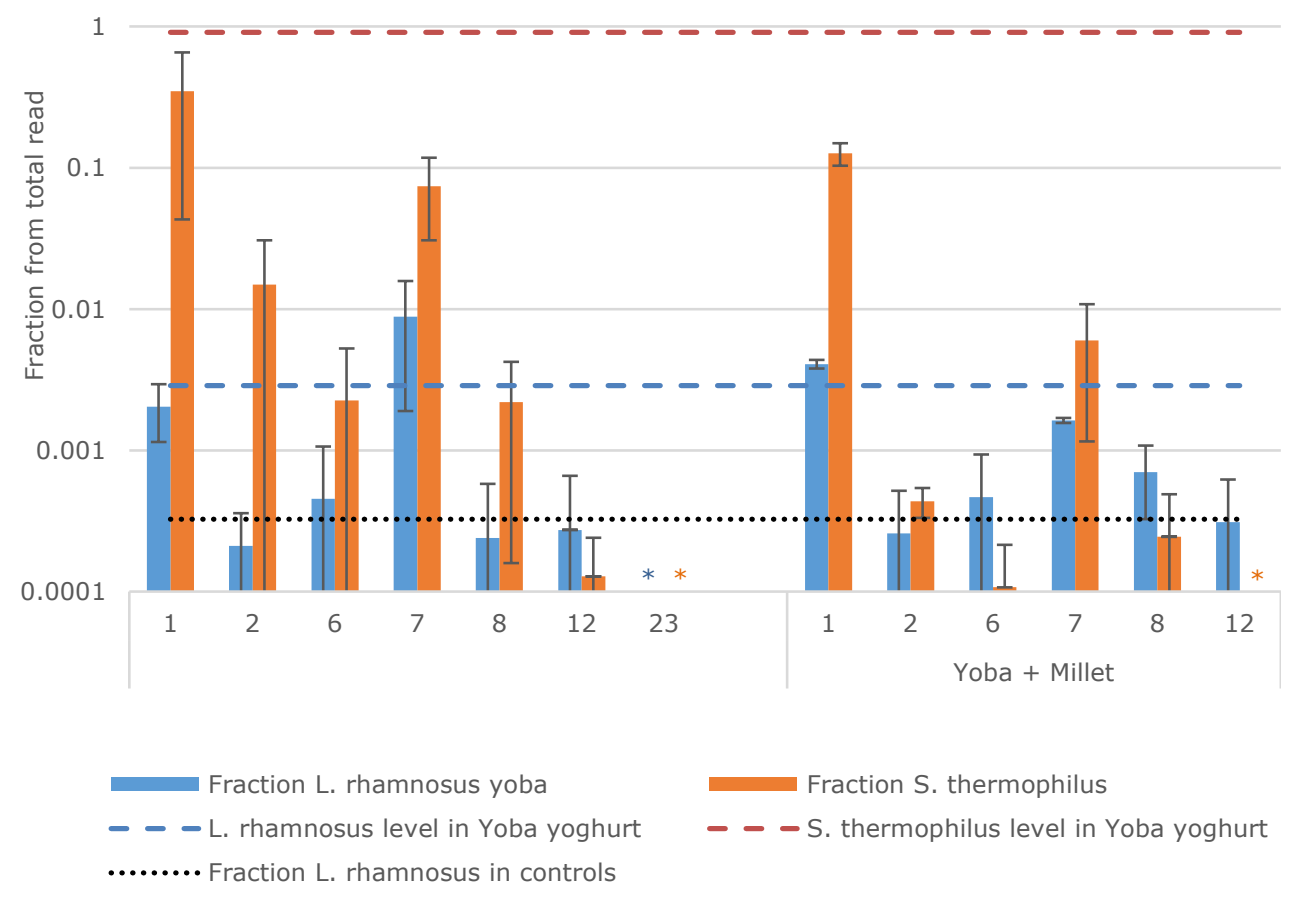

Figure 6.4 Fraction of S. thermophilus and L. rhamnosus in total microbial community in Lait caillé. Yoba: Yoba starter culture addition in lahal 3, 6 and 7 at time points 1 and 7. Millet: Yoba starter culture and millet porridge addition in lahal 2 and 8 at time points 1 and 7 and addition of millet porridge at all 12 time points. The striped lines show concentration of $\mathrm{S}$. thermophilus (orange) and $\mathrm{L}$ rhamnosus Yoba (blue) in the Yoba yogurt (produced in standard sterile conditions). The dotted line shows L. rhamnosus Yoba levels found in controls. * indicates samples where invader levels were below the detection limit. Standard error is indicated with error bars. 


\section{Influence of Yoba starter on bacterial composition of Lait caillé}

After the invasion experiment, the community structures of these five lahals were analyzed. There was no significant change in the Shannon index following the Yoba culture addition with or without millet porridge (Figure 6.5). Nevertheless, we see a trend of increased bacterial diversity as a result of the addition of Yoba starter culture. In the lahals where millet was added along with the Yoba starter culture, this effect seems to be greater. Possibly some strains benefit from the disturbance caused by the invaders and the addition of millet which can lead to greater diversity.

2.50

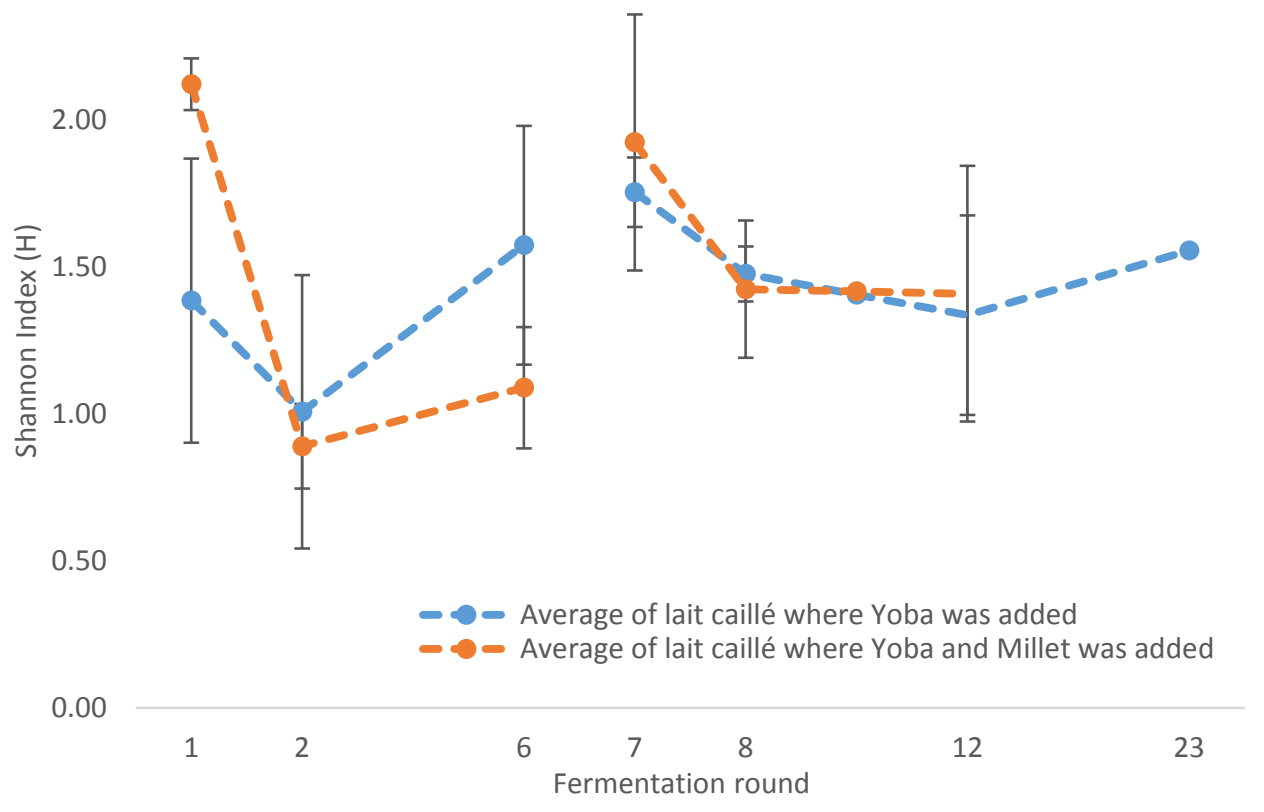

Figure 6.5 Microbial diversity in Lait caillé during and after the addition of the Yoba starter culture. Average Shannon index of lahals inoculated with Yoba (lahals 3,6, and 7) and lahals inoculated with Yoba starter culture in combination with millet porridge (lahals 2 and 8). Error bars indicate standard deviation. Yoba starter culture is added to all lahals at fermentation round 1 and 7 , millet was added to lahals 2 and 8 at all fermentation rounds (fermentations rounds $3,4,5,9,10,11$, and 13 up to 22 were not measured).

Upon the addition of invaders, the community structure of a lahal can change (Figure 6.6). The increase in one species can cause the abundance of another species to change (197). Because the lahals all contain a different microbial composition in the biofilm, also the effects of the Yoba starter culture can be different. As expected, we see an increase in $S$. thermophilus in fermentation rounds 1 and 7 . In addition to a diversity change, in lahals 6 and 8 we observe a decrease in L. helveticus. Without the addition of the Yoba starter culture, this strain has the highest abundance. Besides this change, the addition of Yoba starter culture seems to influence the presence of Acetobacter species. In lahal 6 the addition was followed by an increase in Acetobacter orientalis and Acetobacter tropicalis. As the results shown are all relative abundances, 
we cannot conclude whether the increase or decrease of a certain species was also absolute. Comparing the community structures of lahals before and after the second addition of Yoba starter culture at fermentation cycle 7 reveals that the specific bacterial community of these lahals does not change greatly upon the addition of Yoba starter culture. Aroma composition analysis and other sensory analyses can be carried out to determine whether this translates to stable product characteristics.

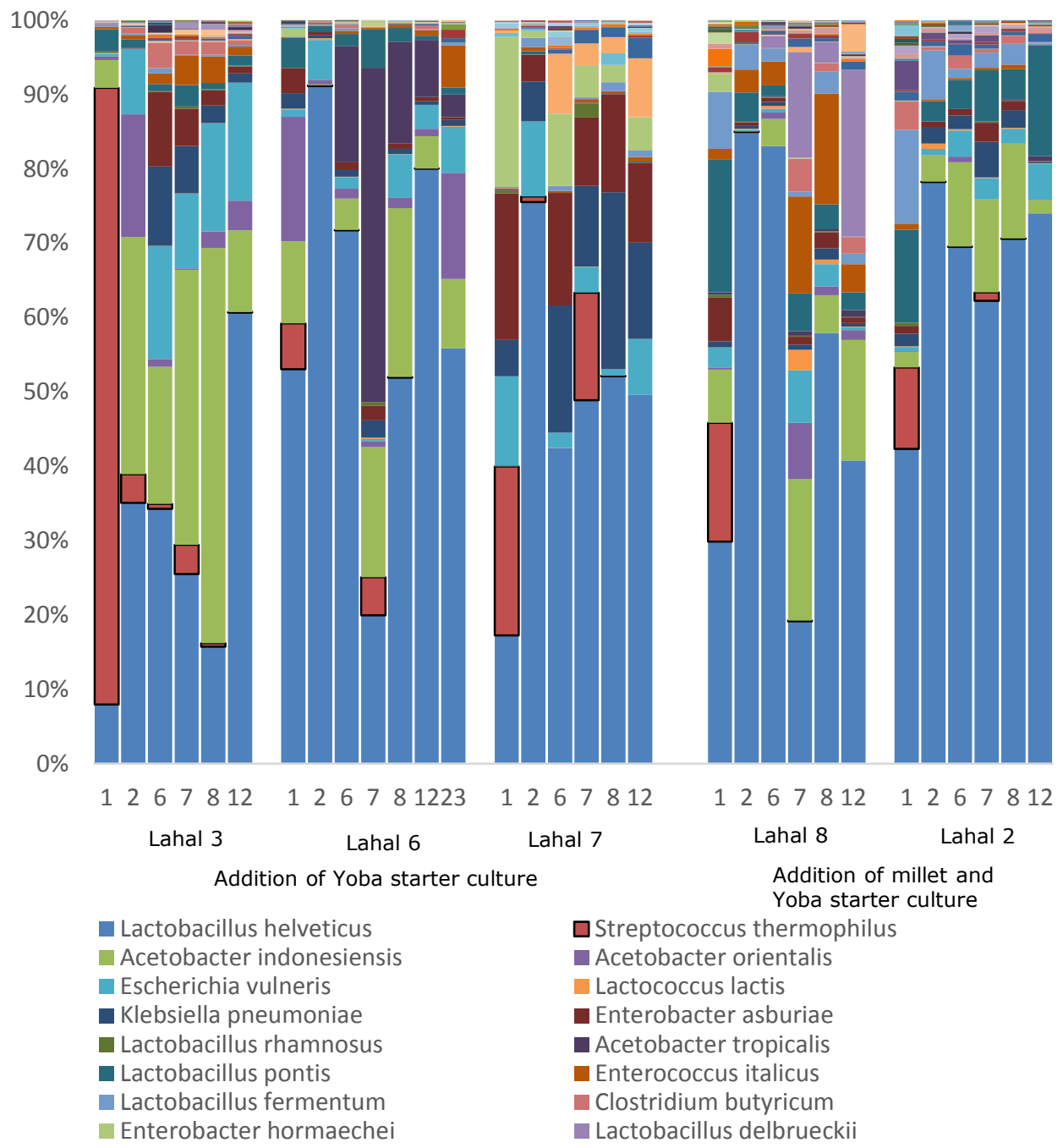

Figure 6.6 Community composition of the bacterial species in Lait caillé of lahal 2, 3, 6, 7, and 8 during the invasion experiment with Yoba starter culture. Numbers indicate fermentation rounds. Vertical axes indicates percentage of total reads within the sample. The thirteen species with highest abundance are indicated in the legend. The aim of the figure is to show the diversity found in the microbial communities of Lait caille in terms of genus and species variation. For specific abundances of species and OTU, please refer to the sequencing data provided with this publication. 
Overall, both strains from the Yoba starter culture could grow in the lahals and remained present in the resulting Lait caillé. The values of L. rhamnosus Yoba in Lait caille are similar to those found in standard Yoba yogurt. The values of $S$. thermophilus are lower than in standard Yoba yogurt. As other bacteria in the traditional Lait caillé community can perform the proteolytic activity, the presence of $S$. thermophilus might not be essential.

The strains in the Yoba starter culture were not able to become members of the Lait caille community remaining in the lahals and were unable to successfully invade the biofilms. The strains might not be able to become part of any biofilm, due to their own characteristics pertaining to biofilm formation; and/or they were not able to become part of these specific biofilms due to a combination of competition with other bacteria and environmental factors. The diversity found in the lahal communities is very high. This decreases the possibility that an invader will find an unoccupied niche where no competition is present, or a niche in which it is better adapted than the original inhabitant. To successfully invade, the invader either must take over the niche of another player in the community or break up the stability of the community by interfering with existing stable interactions. For the added strains their niche in the biofilm is unknown. Species of the Lactobacillus and Streptococcus genera are present in the biofilm, which suggests the possibility for the added Yoba starter strains to become part of the community. However, the strains found in the lahals most likely have had many generations in that environment giving them the opportunity to optimally adapt to their environment. Furthermore, the growth rate of the starter culture is much lower than the growth rate of the original bacterial community in the lahals. Although we added the Yoba starter culture in very high amounts, the time the starter strains were given to adjust to the environment might have been insufficient to for them to outcompete the traditional community. The chance of successful invasion might be increased by selection of Lactobacillus rhamnosus Yoba that have gained the ability to form a biofilm without losing their probiotic characteristics due to genome instability (131). Another solution can be sought in back-slopping higher amounts of Lait caillé produced with Yoba starter culture or standard Yoba yogurt. Alternatively, probiotic strains originating from the natural community of Lait caillé could be characterised, isolated and used to enhance the probiotic properties.

\section{Scientific relevance}

We studied bacterial community dynamics inside wooden lahals over time to observe the naturally occurring variations in community composition. In batch fermentations the fermenting bacteria undergo nutrient cycles. Nutrient levels decrease from a high level while the environment becomes more harsh, due to a lowering of the $\mathrm{pH}$. Nutrient cycling occurs often in nature, for example due to tidal or seasonal changes or in predator-prey interactions. As research on community dynamics is often performed using synthetic communities (70), adding an element of natural variation can give further insight into bacterial population dynamics. In this study, we demonstrate that natural variation is maintained in Lait caillé over several fermentation rounds, while no bacteria are added to the lahal. The lahal therefore must contain the inoculum for the Lait caillé, even though the microbial communities found in the lahals do not show great similarities between to the microbial communities of the Lait caille they produce. 
Aside from nutritional benefits, the addition of a probiotic strain to an existing natural community can give insight into bacterial community stability and resilience. Spontaneously fermented milk products can serve as a model system for research into microbial ecology and evolutionary adaptation. In this study, we see that the initial Shannon index is not a good predictor of the success of invasion of the Yoba starter culture strains. This is not in line with the expectation that a higher initial microbial diversity comes with fewer unoccupied niches and therefore a lower chance of successful invasion into the community (100). Most likely, natural communities have such diverse structures that the Shannon diversity alone is not an accurate indication for invasion success. Other factors, such as the presence of certain bacterial strains or interaction networks between species, might be more relevant indicators. Stability of the microbial community might result in the stability of product characteristics like taste, nutritional composition, and safety.

\section{Societal relevance}

Lait caillé can successfully be enhanced to a probiotic product when Yoba starter culture is added at the start of every fermentation cycle. The addition of Yoba starter culture can result in a product with different organoleptic properties and may have positive effects on the health of consumers in Senegal (120). Probiotic strains can positively influence the composition of the gut microbiota, leading to diverse health benefits, especially for vulnerable groups (198). In regions with widespread undernutrition, increased consumption of traditional fermented food products can provide an opportunity to improve health.

The lahals used in this study are very important for the households or families that owns them. Typically, lahals are a wedding gift and provide a household with a unique, personalised Lait caillé. Due to this, using traditional Lait caillé to enhance nutritional standards is valuable as well as risky. It is valuable because this product is widely consumed and accepted, especially compared to other probiotic products. Other products may be too expensive and/or not well accepted by consumers (83). In contrast, enhancement is also risky because the producers could fear unwanted changes in the product characteristics and be unwilling to change their production method.

To protect the original organoleptic properties of the Lait caillé, changes to the microbial community should be kept to a minimum. Other important attributes to local fortified products are safety, cost, and ease of local production. Due to the potential health benefits of probiotic enhanced Lait caillé, producers of the enhanced Lait caillé might also benefit economically. However, the addition of probiotic strains will change production methods and may increase costs. The addition of a starter culture in every fermentation cycle might not be feasible for a sustainable business model in low- and middle-income countries. Ideally, this probiotic bacterium should be able to survive and proliferate in the natural microbial community. Spontaneous fermentation has lower costs and can result in product characteristics that cannot be attained by fermentation with one or two strains in a starter culture (199). 


\section{Limitations}

Bacterial communities in the lahals are unique. This causes the reproducibility of the experiments performed in this project to be relatively low. To have the ability to perform the experiments in duplicates, pieces of the same lahal which include a biofilm could be distributed over different sterile fermentation vessels. In this way inoculating various fermentations with the same starter.

The DNA analyses method used was only able to detect a wide variety of bacterial DNA. However, it is very likely the microbial community consists of different classes of microorganisms such as yeasts and viruses. Also, this method does not in all cases allow determination of bacteria to a subspecies level. Other techniques, such as mRNA analyses and PCR techniques using specific primers for target organisms, such as lactic acid bacteria, could be used to get a broader view of the total community present in the lahals and the Lait caillé.

\section{Conclusion}

In conclusion, we found that Lait caillé harbors a diverse bacterial community of lactic acid bacteria that is stable in terms of composition over multiple rounds of fermentation. Although the biofilm of the lahals is the only inoculum for the Lait caille produced in this study, the bacterial composition of Lait caille differs from that in the biofilms as some strain in the biofilm grow much better in milk than others. The addition of Yoba starter culture to every fermentation cycle of a spontaneously fermented milk product like Lait caillé can result in a probiotic product. It is remarkable that even a diverse community such as the bacterial community found in the lahals allows for the temporary inclusion of another species. This knowledge can inform further research aimed at leveraging the benefits of other traditional and widely consumed products supplemented with Yoba starter culture. Future research on traditionally-produced Lait caillé may generate evidence of probiotic properties of the microbiota in it and hence of Lait caillé itself.

\section{Acknowledgements}

We gratefully acknowledge the families in Senegal who provided us with their family lahal. The help of Joost van den Heuvel in the analyses of the sequencing data is highly appreciated. Finally, we thank Connor Edick for critically reviewing the manuscript. 


\section{Supplementary material}

Supplement Table 6.S1 Community structure of wall scraping of lahals upon arrival in the laboratory.

\begin{tabular}{|l|l|l|l|l|l|l|l|}
\hline Iahal & original & & \multicolumn{3}{l|}{ Blast } & \multicolumn{3}{l|}{ Shannon index } \\
\hline & & \#OTUs & \#Species & \#Genera & OTUs & Species & Genera \\
\hline 1 & 2 & 237 & 87 & 47 & 3.20 & 2.57 & 2.06 \\
\hline 2 & 3 & 122 & 43 & 30 & 3.05 & 2.20 & 1.74 \\
\hline 3 & 4 & 112 & 49 & 35 & 2.83 & 2.06 & 1.66 \\
\hline 4 & 6 & 70 & 28 & 18 & 1.98 & 1.20 & 0.87 \\
\hline 5 & 7 & 65 & 18 & 7 & 2.03 & 1.19 & 0.47 \\
\hline 6 & 8 & 73 & 27 & 18 & 2.24 & 1.22 & 0.78 \\
\hline 7 & 9 & 90 & 29 & 16 & 2.35 & 1.27 & 0.42 \\
\hline 8 & 0 & New lahal, not previously used & & & \\
\hline
\end{tabular}

Supplement Table 6.S2. Details available of the origin and previous use of lahal 1 to 7 . Hierarchical cluster tree is based on OTUs of the biofilm samples of the lahals.

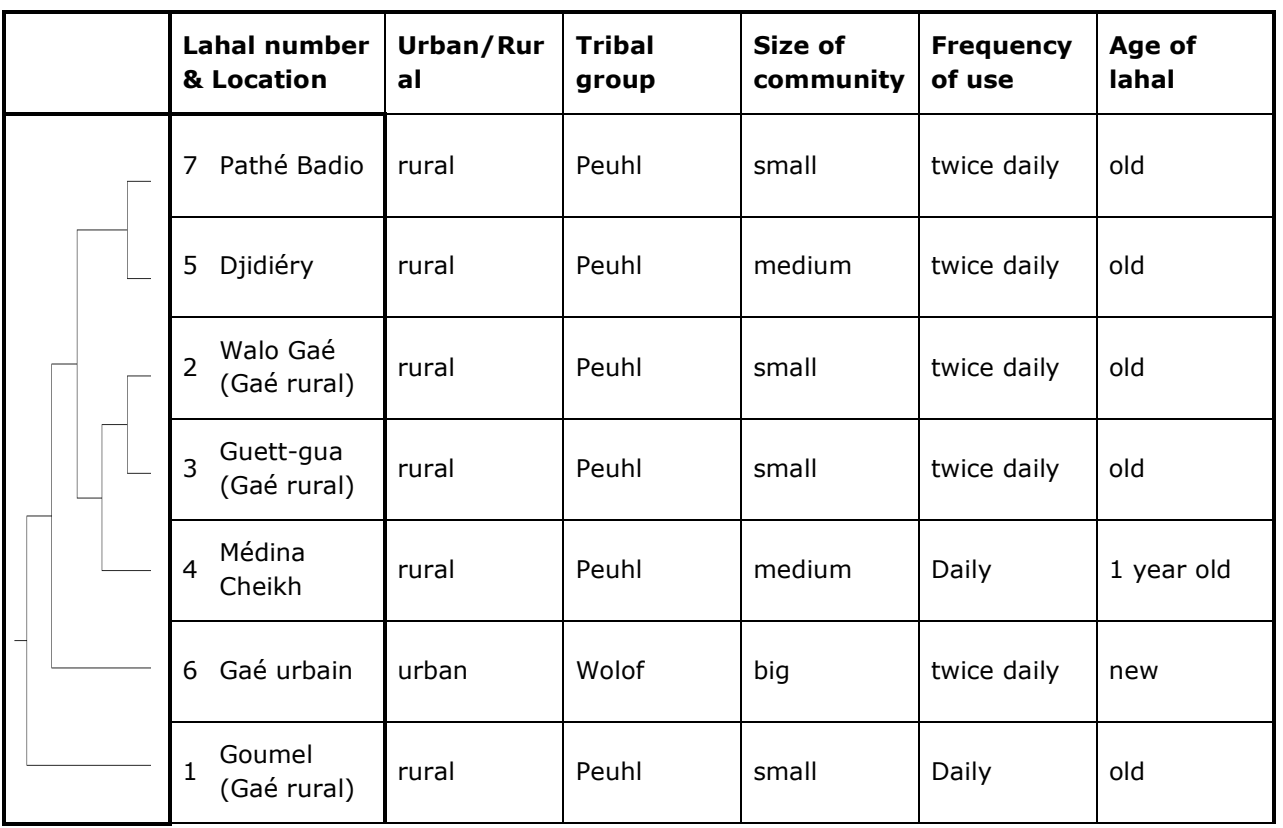


Chapter 7. 


\section{General discussion}

Anneloes E Groenenboom 


\section{Introduction}

In this thesis a first step is made towards the use of microbial communities from spontaneously fermented milk as model systems for ecological and evolutionary experiments. These communities are unique model systems as they represent a natural community with limited complexity and a clear function. Working with natural communities has its challenges, and while I have overcome several of these, after completion of this thesis questions remain. In this general discussion I reflect on what we now know about bacterial communities from Mabisi and Lait caillé and their community dynamics. Moreover, I share my ideas about how to interpret adaptations and diversity in natural communities. The introduction (Chapter $\mathbf{1}$ ) to this thesis and Chapter 2 highlighted a list of research questions. My thesis described fundamentbuilding experiments that are essential for future investigations. In this general discussion I give suggestions for further work, both towards a better understanding of eco-evolutionary dynamics and towards applied research in the field of science for development.

In the introduction I presented three disciplines, food fermentation, ecology, and evolution, which I combined in this thesis. The disciplines can now be further specified to represent the work that was performed (Figure 7.1). From the side of food fermentation, I focussed on the spontaneous fermentation of two traditional African dairy products, Mabisi and Lait caillé. The bacterial community structure of these dairy products, in terms of species composition and diversity, was the main ecological characteristic analysed. Changes in species composition over time under the influence of various biotic and abiotic changes in the environment was the first step towards analysing evolutionary influences on bacterial community structure.

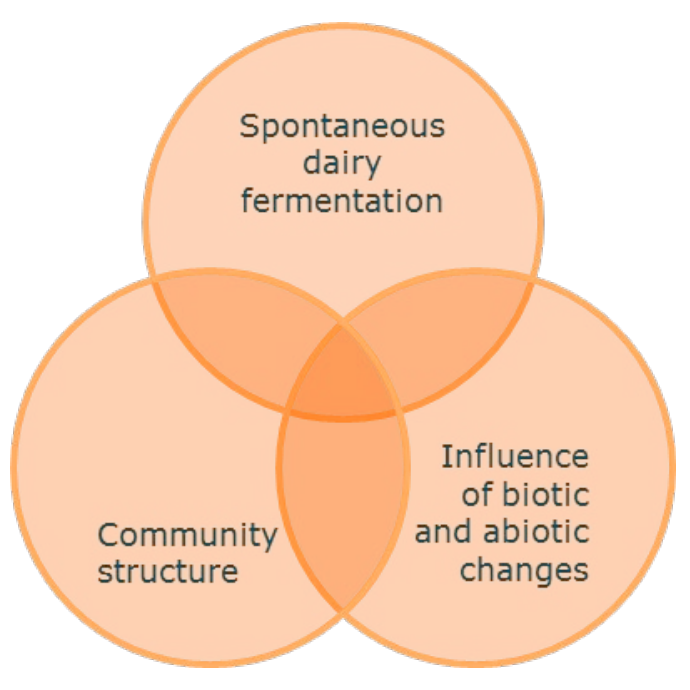

Figure 7.1 Linking three disciplines in one study. Adapted figure from the introduction; the three study fields are defined more specifically here. Food fermentation is narrowed down to spontaneous dairy fermentation, ecology to community structure and evolution to influence of biotic and abiotic changes. 


\section{This thesis}

\section{Main findings and conclusions}

This thesis starts with a perspective on the use of microbial communities of spontaneously fermented products as model system for eco-evolutionary dynamics (Chapter 2). In the first part of this perspective we outline what model systems have been used for experimental testing of evolutionary theory so far, ranging from simple microbial communities in the laboratory and, more recently, to complex (natural) communities. In the second part we explain what research questions with an evolutionary background can be addressed using these microbial communities from fermented foods. These microbial communities are a suitable model system to study eco-evolutionary dynamics as they combine the complexity of a natural community with the ease of analyses of a synthetically defined community.

The focus of this thesis is on the bacterial community of spontaneously fermented Mabisi. As a first step the natural communities of this traditional fermented product were analysed in a field experiment (Figure 7.2). A method was developed using filter paper disks for the preservation of DNA from diverse microbial communities for further analyses on a later moment (Chapter $\mathbf{3}$ ). The microbial community structure obtained via the filter paper method showed sufficient resemblance to the structure obtained via traditional DNA extraction from the liquid milk sample. This method could successfully be used to analyse diverse microbial communities from Mabisi in our remote field sites in rural Zambia. Field experiments were conducted to determine the effect of variations in fermentation vessels and types of back-slopping procedures on acidification and microbial community composition during fermentation (Chapter 4 ).

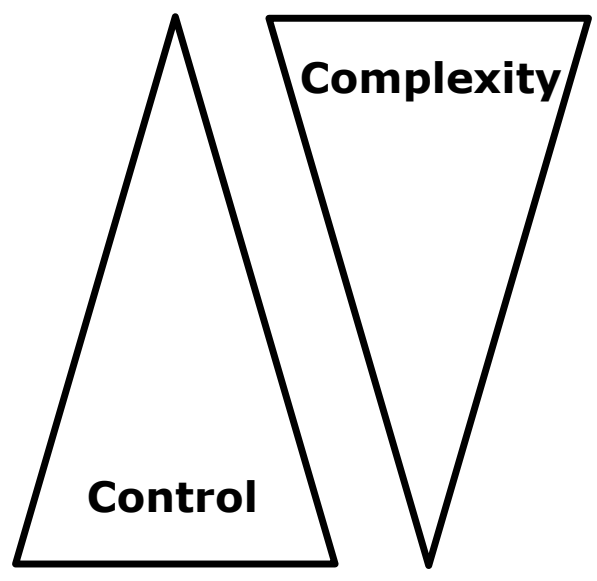

(Complex natural systems)

1) Traditional fermentation $>$ Chapter 4

2) Natural communities in the lab

$>$ Chapter 5

3) Synthetic ecosystems

(Single strain experiments)

Figure 7.2 Set-up of experiments to cover translation between single strain experiments and complex natural systems. Including thesis chapters. 
Due to high costs and a reduced availability of the traditionally used calabashes, nowadays more and more plastic containers are used for Mabisi production. However, the effect of this change in production practice on the properties of the product is unknown. In our work no significant difference was found in terms of microbial diversity during and at the end of fermentation between fermentations performed in buckets or previously used calabashes. In case of "active back-slopping", the pH drop started right after inoculation. In the "no back-slopping" and "passive back-slopping" fermentations, there was a clear lag phase before acidification started. All experimental series resulted in a microbial community dominated by Lactococcus lactis. After the natural communities of traditional spontaneous fermentation were analysed, the second step was to bring the natural communities to the laboratory. Here, experiments were performed with lower complexity and an increased level of control (Figure 7.2). Species composition and metabolic profile of six comparable but different Mabisi samples were analysed before and after repeated propagation cycles (Chapter 5). These starting communities had high similarity in their bacterial species composition and therefore had the potential to converge towards the same final species composition upon repeated propagation in a common environment. However, at the end of the repeated propagation cycles, communities were either dominated by Lactobacillus helveticus or by Lactobacillus delbrueckii. Replicate communities that are from the same ancestral community changed in a similar way in response to the new environment. By modelling hypothetical species compositions, we tested the influence of four main factors on the species composition: initial species composition, selection imposed by the environment, change caused by interaction between species and random processes in species dynamics. We found that the final species composition is mostly dependent on initial species composition, followed by random processes. The environment showed to have the least influence on the change in species composition.

We had the opportunity to work with a second spontaneously fermented dairy product due to a collaboration with Path, an NGO from Seattle, USA. This traditional product, called Lait caillé (Figure 7.5C), originates from Senegal and is produced in wooden bowls, called lahals (Chapter $\mathbf{6}$ ). In terms of complexity and control, this experiment would position in between step 1 and 2 (Figure 7.2), as traditional fermentation vessels were used, containing natural biofilms with bacterial communities. The experiments were performed in the laboratory. Therefore, many variables, such as temperature and time in the fermentation process, could be controlled. However, the mode of propagation was traditional, meaning that the bacteria were transferred via the inside of the lahals that were used for repeated fermentations. This decreased the control compared to the laboratory experiment with Mabisi (Chapter 5), where glass bottles were used. This method also made replication impossible; the microbial community within one lahal is not the same as the microbial community in another lahal. Nevertheless, the traditional production method allowed us to study the natural communities in the lahals and translate our findings to production practices in Senegal. We analysed the species composition of Lait caillé over time and added a probiotic starter containing Lactobacillus rhamnosus yoba 2012 to the traditional process in an attempt to enrich the bacterial species community in the final product. We found detectable levels of $L$. rhamnosus in the final product, ranging between 0.2 and 1 percent of the total bacterial population, which is comparable to the concentration found in probiotic yoghurt. Subsequent rounds of fermentation using passive back- 
slopping without the addition of new L. rhamnosus led to a loss of this strain from the community of fermenting bacteria. Just as Mabisi it is an important part of the daily diet of men, women, and children in rural and urban areas. The addition of a healthpromoting, probiotic bacterium to these products ensures the delivery of that probiotic activity to consumers. The addition of probiotic strains at every fermentation cycle can enrich the existing complex communities of traditionally fermented Lait caillé while traditional bacterial strains remain dominant in the bacterial communities.

\section{Microbial communities of spontaneously fermented milk}

Although coming from the same product, the microbial communities found in different Mabisi samples show clear variation. Where in the field experiment (Chapter 4) the finished Mabisi is clearly dominated by an OTU that was identified by BLAST as a Lactococcus lactis, in the laboratory experiment (Chapter 5), the communities at the end of fermentation had a more even distribution and were dominated by one of two OTUs that came out of the BLAST as Lactobacillus helveticus and Lactobacillus delbrueckii.

Lactococcus lactis is known to be mesophilic (200), while L. helveticus and L. delbrueckii are thermophilic (201). Therefore, the difference we see in the microbial communities could be caused by differences in fermentation temperature. Indeed, the temperatures in the field experiment (Figure 4.2) are on average below $28^{\circ} \mathrm{C}$, which was the set temperature in the experiment in Chapter 5. Also, temperature fluctuations in the field fermentation may have significant influence on the community structure given the mesophilic character of L. lactis. Probably these three species fulfil about the same ecological function within the community, but OTU data do not provide enough detail to determine the exact particular species function in the community. If these two species have the same function in a different community, Lactococcus lactis leaves less ecological space for other species to become more abundant. In the communities where $L$. helveticus and $L$. delbrueckii have the highest abundance, we see an more even distributed species composition, indicating that $L$. helveticus and $L$. delbrueckii might be more successful in a relatively low abundance. Alternatively, these three species compositions could represent three robust clusters in Mabisi communities. This observation might be analogous to the enterotypes that can be identified in the human gut microbiome (202).

Another cause of the difference might be the variation in experimental set-up, such as sampling method or the moment of transfer. Due to dependencies in the communities, the moment of transfer can have a substantial influence on species sorting during community propagation (Figure 7.3). In case of milk fermentation, there is often a succession of bacteria during the process, where bacteria that have a high growth rate dominate at the beginning of fermentation, while others grow on a later moment, as they use the metabolites of the first bacteria as resources $(12,27)$. An example is the abundance of acetic acid bacteria (AAB), as these bacteria gain their energy from metabolising fermentation products, such as lactic acid (203). AAB only start growing at a later stage of the fermentation cycle after lactic acid bacteria (LAB) have produced lactic acid. Doing transfers at the early stages of the fermentation process (i.e. the moment that $L A B$ are proliferating) will select for $L A B$, while transfers at a later stage will select for $A A B$ as well as other bacteria that can remain in acidic environments. 
This effect of preferential selection during propagation can also happen between LAB, when there are dependencies at the level of metabolite secretion or alternatively due to different levels of acid tolerance. The result is that the moment of transfer determines the bacteria that are preferentially transferred, i.e. the bacteria that will be selected for. Possibly the number of Lactococcus lactis was higher after 24 or 48 hours of fermentation in the laboratory, but as the transfers were only done after 84 hours, the relative abundance of $L$. lactis might have decreased already, increasing the relative abundance of other species, including species that start growing later and species tolerant to high acidity.

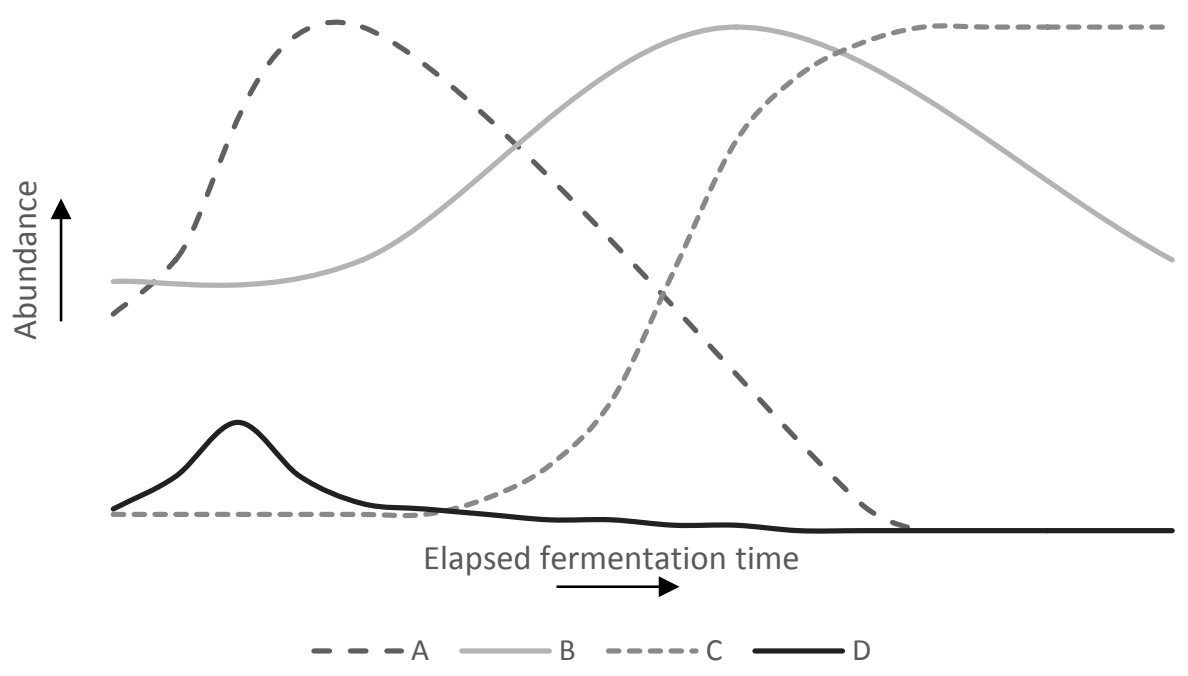

Figure 7.3 Transfer moment determines species selection in a hypothetical fermenting community. Elapsed fermentation time from the start of fermentation of raw ingredients. Fast growers will increase in abundance at the beginning of fermentation $(A)$. Other species can use the metabolites of the fast growers as nutrient source ( $B$ and $C$ ). Increased acidification over time can cause some species to decrease in abundance ( $A$ and $B$ ) while others can persist in acidic environments (C). Some species might have a very narrow moments of proliferation (D), which makes the moment of transfer an essential factor in species selection and community composition, as observed by Spus et al. (unpublished).

The largest difference between the production of Mabisi and that of Lait caille is the treatment of the milk prior to fermentation. Where in Lait caille the milk is boiled leading to almost sterile environment before fermentation commences, in Mabisi raw milk is used and the natural communities present in the milk are still present at the start of fermentation. This makes it possible to perform the fermentations in clean vessels, such as plastic buckets and bottles or metal cans. Even without the inoculum of the passive back-slopping via the inside of the calabash, the bacteria present in the milk will start the spontaneous fermentation process. When pasteurised milk is poured in the clean vessels, the initial acidification rate might be too low, allowing invaders to 
spoil the milk and cause food safety issues. Assuming that the microbial communities in lahals and calabashes have co-evolved, they have done so in different environments. Where the influx of new bacteria in the lahals is minimised and the community present in the lahal can co-evolve without new competition, the raw milk added to the calabashes can contain many bacteria that will compete with the co-evolving community. This difference in the level of competition could have an effect on the coevolution in both environments. The communities of Mabisi might be more evolved to compete for resources and produce antimicrobials. The Lait caillé community might have co-evolved stronger interactions and the ability to grow in milk of higher temperatures.

Strong co-operation and dependencies within microbial communities might be a poor evolutionary strategy as it makes a species dependent on another and therefore more vulnerable to changes (21). Competition might be the main interaction in microbial communities and cause it to stabilise $(204,205)$. However, due to the complexity of the resources in milk, bacteria might need to use each other's metabolites to increase their growth, as is the case in many fermentations $(206,207)$. As the communities in spontaneously fermented foods have been together for long periods of time and in a relative stable transferring system, the evolution of collaborations might be more prevalent compared to other natural systems, which experience more fluctuations such as the human gut and the soil.

Some of the pilot experiments that were performed during this study suggested that the addition of peptides made the proteolytic bacteria redundant and disappear from the community. When the metabolites produced by a certain species are not needed by others anymore, these bacteria cannot maintain themselves in a community. This raises the question whether species could protect themselves against this, in order to have a stable existence in a variable environment. In these explorative experiments we tested these ideas using a synthetic community, meaning that these bacteria were artificially combined in this community. The bacteria did form a stable community in normal milk fermentation but did not co-evolve as the natural communities in Mabisi could have done. During co-evolution, multiple interactions could evolve, safeguarding the species' presence in the community. Also due to the low diversity (a combination of five species), the community might be less stable compared to natural communities.

\section{Community adaptation, is there such a thing?}

The changes we observed in microbial community structure, diversity, and composition when a community is brought in a new environment, are sometimes describes as community adaptation. However, the meaning of adaptation can be differently interpreted by scientists from different areas of biology. Nowadays the focus of our research is more and more on combining different fields of biology to come to a complete understanding, such as the combination of ecology and evolution. Clear communication, including an encompassing definition per concept, is necessary in multidisciplinary research (proposition in (208)). Deciding what this one definition will be, especially in words that are embedded in various research fields as well as in society, is challenging nevertheless very much worth trying and do-able. 
When looking at the results of Chapter 5, one might assume that the communities in their new environment adapted towards a species composition that is most beneficial for the community - where most of the available nutrients can be converted to energy. For Mabisi in this particular environment, we seem to have two ecological states in which nutrient utilisation would then be most optimal. This is very similar to a fitness landscape (64) with two optima, where communities have a species distribution towards one of the two optima dependent on their origin. Defining adaptation as the ability to survive and multiply due to natural selection acting in random genetic variation makes that communities cannot adapt, as they cannot multiply as a whole. Although, through interference of the producer, some communities can be used for further fermentation while others are discarded due to unwanted characteristic, such as the production of an off-taste. Taking adaptation in a more general context, adaptation can include changes in function, structure, or behaviour by which the chance of survival in a certain environment is maximised. Possibly this is what happened in the experiment in Chapter $\mathbf{5}$.

A trait in one species cannot be selected solely for the benefit of another species. However, in a community with interactions, a trait of a species might be a result of its interactions with other species' traits. Therefore a part of the genes that are responsible for a specific trait might not be present in the species that has the certain trait, but in another species (33). Selection in this case is not on the individual that carries both the trait and has the benefit. In this way the selection is not only at a species level but at a higher level, at least those of the two species combined. Assuming there are many metabolic dependencies in the community, selection might be on the whole community (Figure 7.4). Also the interference of the Mabisi producer changes the selection pressures on the community.

When combining ecology and evolution, a separation is needed to connect what we observe in the community in terms of shifts in species composition to underlying dynamics caused by changes in individual species (evolution) and by ecological interactions (and changes in those interactions) (209). We made a start in dissecting which factors influence changes in a community over time in Chapter 5. To find out details about what share is caused by evolution, we need to sequence our bacteria and see what mutations we see in the community and whether they are similar in all communities.

\section{Community diversity and its functions}

For industrial fermentation of milk to yoghurt only two species of bacteria are necessary. Nevertheless, communities in spontaneously fermented milk are very diverse. This diversity is possibly a result of the selection pressures on the community by the producers as it can have an influence on product characteristics and functional stability.

In this thesis (Chapter 4, 5 and 6), I analysed the Shannon Index of various communities as a means to compare their relative diversity. Moreover, the analysis was done to assess effects of the treatments used to obtain a sense of the stability in terms of species composition and abundance in the communities. 
A

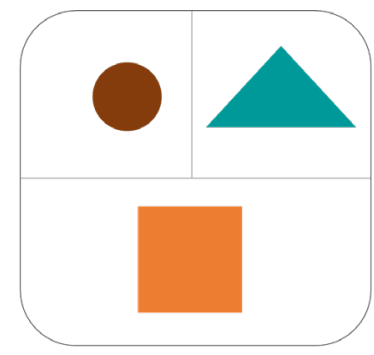

B

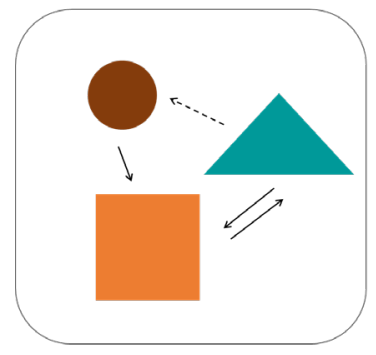

$\mathrm{C}$

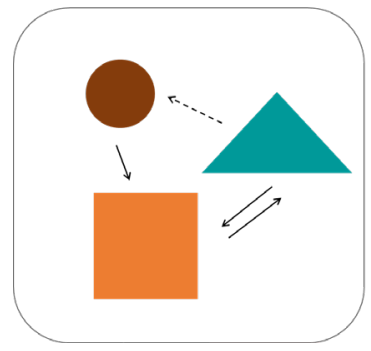

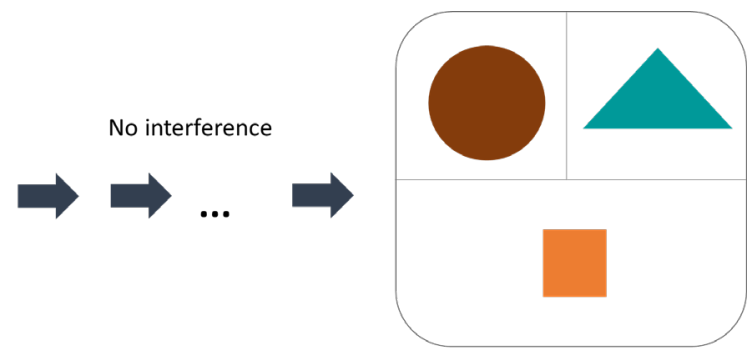

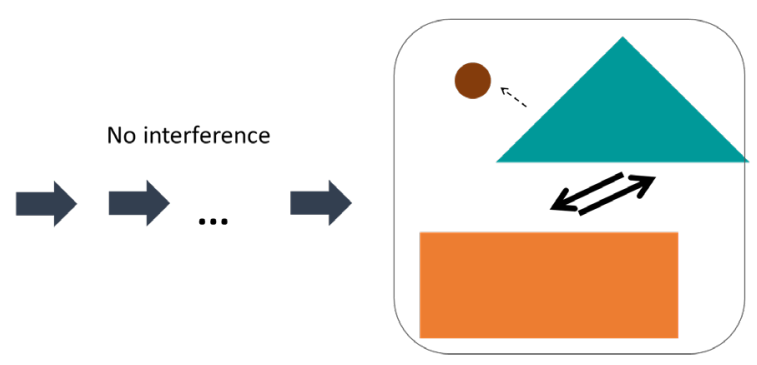

Producer controls quality

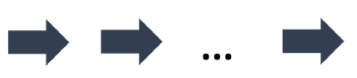

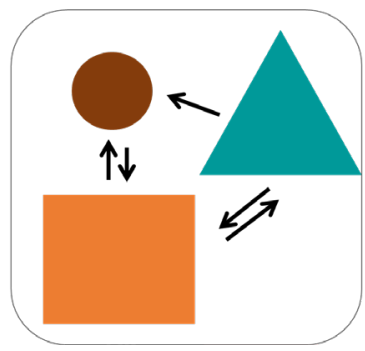

Figure 7.4 Influences on adaptation during production of spontaneously fermented food. The cartoon shows hypothetical eco-systems, composed of different bacterial species represented by the different shapes. Arrows within the community represent interactions. Thick blue arrows represent repeated propagation cycles. Bacteria adapt to the same environment in repeated propagation cycles, without (A and B) or with (C) interference of the producer to ensure a certain metabolic output (preferred product characteristics). Panel A shows how single species in isolation will adapt to a (new) environment, by optimizing the use of available resources. Panels B shows how in communities the biotic environment can be shaped by interaction between species, having an influence on an individual. Upon repeated propagation cycles, individual species may adapt to the conditions, which will not only lead to more efficient growth of some individual species, but to an increased productivity of the overall community. In communities where a producer interferes and acts as a quality controller, this interference may alter the selective pressures towards species adaptation within this community. One might expect differences in final community structure and interactions due to a combination on selection on bacterial growth rate and effective use of available recourses and selection on a community that produces a product with preferred characteristics, such as a pleasurable aroma and viscosity. 
Overall the number of different species present in these communities remained at the same high level throughout the experiments. We experienced a slight drop in bacterial species diversity during fermentation (Chapter 4) or during repeated propagation cycles (Chapter $\mathbf{5}$ and $\mathbf{6}$ ). The total collapse of a community, where only a few species were left while the Shannon Index decreased towards zero, was not observed in any of the experiments. This is an understandable outcome of the repeated propagation cycles, as they were performed in a relatively simple environment. The maintained diversity can be explained by negative frequency dependence, where a species with the highest abundance is also most likely to have enemies such as bacteriophages. This is also referred to as the 'kill the winner' principle $(3,101)$. Stability in a certain diversity can also be a necessity of a system because the addition of species as well as the removal of species can destabilize the system due to the (evolved) bacterial interactions (210).

Community diversity is in many studies directly linked to stability, where stability is defined as resilience of function and diversity against perturbations (see Chapter $\mathbf{2}$ ). The diversity we find in Mabisi, Lait caillé, and other spontaneously fermented products is much higher than in the communities frequently used to investigate the diversity stability relationship, which typically add up to a few dozen species (55). It might well be that at this level of diversity the exact number of species has no influence on community function (17). A minimal community could be constructed, where all redundant species and functions are removed, analogues to the construction of a minimal cell (211). When constructing a minimal community that should represent the characteristics of the diverse and stable microbial community, the individual effects of species on community functioning prevail and should therefore be selected carefully (212). In this context, diversity in itself might not be meaningful. Rather both diversity and function are a result of a similar cause, namely presence of species or functional groups $(98,213)$. Also, there can be a difference between compositional diversity and functional diversity (193). When we see diversity as a starting point, we can use it to continue to investigate community function. Microbial diversity is only the beginning.

\section{Challenges}

Conducting experiments using natural communities, in the field and in the laboratory, results in many uncontrolled variables. This might present a challenge and raise questions at the end of an experiment when a high degree of variation is observed that cannot be accounted for by the controlled variables. High levels of variation that we cannot explain directly, most likely results from natural variability linked to circumstances. Opposed to being a challenge, useless and annoying, this variation can increase our understanding of natural variability and its impact on experimental outcomes. That is, just dismissing this variation as a flaw of the experiment would be a loss, as it can contain much biologically relevant information.

The samples of the various experiments described in this thesis, as well as experimental results generated in the entire project group, were obtained by high throughput sequencing in three batches using the same MiSeq Illumina sequencing method. The only difference between these batches was the size of subunits available, resulting in slight differences in number of reads per sample. However, the two batches of sequencing appeared to be incomparable. Samples that were sequenced in two 
different batches did not result in a similar species distribution, and clustering all samples to see methodological effects only resulted in a clustering of the samples from the same batch. This phenomenon is observed more often $(142,167)$, but it is unknown what causes this strong batch effect of MiSeq sequencing. This finding has two consequences for interpreting the data: 1 ) only samples from the same sequencing run can be compared, and 2) differences found between samples of one run only have relative value.

In my studies I focussed on the analyses of bacteria using 16S rRNA gene sequencing as lactic acid bacteria are known to be most abundant and important for milk fermentation. The changes in bacterial compositions analysed in this thesis might be dependent on organisms of other taxa present in the microbial communities (96). Analyses of the presence of other taxa, including species such as fungi and yeasts, should be done to get a broader overview.

\section{Future work}

In my set-up of experiments (Figure 7.2), I differentiate between three levels of complexity, with increasing control and decreasing resemblance with nature. In this thesis I could only work up until the second level. Nevertheless, for the third level, the one with most control and least complexity, I performed some preliminary experiments as well. Here, I will share the results and interpretation derived from these experiments and discuss follow-up research that would build on these results.

\section{Dissecting community functions}

Changes in community structure, such as those observed in Chapter 5, could be better understood, and therefore predicted, when community functions would be known. General functions and capabilities of species such as Lactobacillus helveticus, Lactococcus lactis, Streptococcus thermophilus, and Acetobacter pasteurianus have been extensively studied. However, as there are many differences between subspecies, as well as phenotypic plasticity regulating what metabolic networks are active, the characteristics of similar species in isolation do not translate directly to the specific role of these species in a natural community.

One way to start dissecting community functions is by looking at the volatiles that are formed as a proxy for metabolic activity. In Chapter $\mathbf{5}$ I used this method to analyse the metabolic profile of the full community, but this method can be further extended to analyse contributions of certain bacteria to this metabolic profile. Reconstructed communities with a limited number of species can be used to ferment milk as well as similar communities where one of the species is left-out (single omissions). The difference in metabolic profile of these communities gives information about the role of the omitted species. Another way to assess the functions and activities in the community is to analyse the messenger RNA in the community showing what genes are expressed (RNA-seq (214)). Combining RNA-seq data with DNA sequencing data of a community can give insight into what pathways are active in the community and what bacteria are present. However, when the active genes are present in multiple bacteria in the community, one cannot always pinpoint which species actually has this function. 
The functions in a community are essential for the formulation of a starter culture. This starter culture should produce a Mabisi that is safe, nutritious, and tasty. The bacteria of the culture should form a stable community, even when back-slopping in sterilised milk is performed, where the metabolic output is unchanged over time. This functional stability results in stable Mabisi characteristics. Compositional stability is linked to functional stability and can be used as indication for functionality as it is less complicated to determine. Compositional stability is in itself less important for function, as communities with various compositions can attain the same function and produce Mabisi of similar characteristics. If the starter culture can maintain its functional stability over repeated propagation cycles, this could decrease the cost for Mabisi producers as they do not have to invest in starter culture for every fermentation round.

I hypothesise that it is necessary to form a community with more than two species. In that way the flavour richness of traditional Mabisi can be maintained. Also, a more diverse community can be more stable in terms of resistance against invasions and maintaining function. Pilot studies have shown that communities of up to five bacteria can form a stable community, in which all species are still present at the end of fermentation. This suggests that cross feeding and resource competition can stabilise co-existence in a community. Industrial fermentations with defined starter cultures of more than two species are uncommon. However, fermentations using a multi-species community might decrease contamination and enhance product characteristics (215).

Pinpointing which bacterial species can be designated as key-species - necessary for typical Mabisi characteristics - requires further investigations. Besides a safe and consistent product, the bacterial starter culture for Mabisi should produce the right aroma profile and acidity as well as the right product texture. The desired taste and consistency of Mabisi can differ from consumer to consumer. We might have to produce various starter cultures to address the various wishes of consumers. Bacteria could therefore be selected for one or more of the following characteristics: acidification rate, aroma formation (direct and indirect via excreted metabolites), exopolysaccharide formation and proteolytic activity as well as other stabilising factors such as interactions that can increase or decrease the growth of other bacteria. Species isolated from Mabisi might be the best starting point as those species have adapted themselves to the Mabisi environment by co-evolution. Therefore, these bacteria may have developed the capability to produce the characteristic Mabisi flavour. The outcomes of competition experiments with small sets of species can be used to predict the compositional stability of a more complex system (71). This can be used to formulate a functionally stable starter culture when the necessary functions of the community are known.

Some key species can be present in the biofilm of a fermentation vessel and cause Mabisi produced in calabashes to have its district taste. This might be the reason for Mabisi produced in other vessels to be perceived as less flavourful. As showed in Chapter 4, no clear difference between Mabisi communities produced in calabashes or buckets could be found. The question remains whether the perceived difference is a technical product characteristics or consumer perception. A hypothesis is that these specific bacteria can only persist through passive back-slopping and the biofilm is their "basecamp" (207) from which they grow in the milk. Further analyses of the biofilm 
could shed more light on its function. My research on Lait caillé clearly shows that the biofilm in the lahals provides the inoculum for fermentation (Chapter 6). However, the biofilm community does not represent the resulting Lait caillé community, which is also seen in the species distribution in kefir and kefir grains (207).

\section{Community resilience}

An aspect of community stability is its resilience after perturbations (204). A resilient community has the ability to return to the original composition and function after a perturbation. These perturbations can be of all sorts, from fluctuations in abiotic factors, such as temperature and nutrient availability, towards biotic factors, such as an invader. When an invader enters the environment, it needs to either occupy a vacant niche or take over a niche from another species in order to establish in that community. In diverse communities all niches will be occupied, resulting in a high chance of resilience against invasion. As microbial communities found in spontaneously fermented foods are diverse, they are hypothesised to be rather resilient against pathogenic contaminations.

Preliminary experiments have shown repeatedly that various pathogens are not likely to survive in Mabisi. During Mabisi fermentation, the concentration of pathogens decreased until below detection levels. This could be caused by the acidic environment, production of antimicrobials by the original Mabisi community, a competitive environment due to microbial diversity, or a combination thereof. Further research to ensure the safety of Mabisi should include challenge tests of potential pathogenic bacteria into raw milk at the start of fermentation and the identification of critical control points in the processing together with critical limits. With this information, production can be standardized and formal approval of relevant authorities can be requested. This will allow scaled-up production and sale of Mabisi.

Mabisi is a safe, tasty, and nutritional product and part of cultural heritage. The production of Mabisi is therefore of high importance for the family of the producer, who can consume a filling and healthy product on a regular basis. If the producers would have the ability to produce Mabisi on a larger scale and find a proper market for their product, this could also increase their economic status. However, finding the right market is difficult. Moreover, transport of Mabisi to regional and urban markets is challenging. On top of that, the local authorities (i.e. the Zambian Bureau of Standards) currently do not accept the sale of an unpasteurised milk product, which is mostly the case with Mabisi.

\section{Co-evolution}

As explained in the Introduction and Chapter 2, the microbial communities of spontaneously fermented foods are a useful model system for studies of ecoevolutionary dynamics. Now that we know more about those communities, we can start using them for all sorts of experiments, such as those suggested in Chapter 2. Also, we could repeat the experiment in Chapter $\mathbf{5}$ with more propagation cycles and analyses of active genes and mutations to analyse similarities in species adaptations and determine the proximity towards the evolutionary 'end-point'. The selection pressure on this system could be increased, for instance by minimising the available nutrients, in order to see how selective the environment needs to be to overcome the 
influence of the initial community composition. In previous experiments, as part a of student project, we observed a total collapse of a community, where only one species was left after repeated propagation cycles (unpublished data). Possibly the selection pressures in this experiment were much higher, causing the observed difference.

The same experiment can also be executed with natural Mabisi samples of which the origin is known; produced in calabashes or buckets, using back-slopping or not. This can give insight into how different levels of co-evolution (co-culturing) before the controlled experiment influence the species composition at the end of the experiment. Also diluted natural communities or reconstructed Mabisi communities can be used, in order to study the stability, functionality, and dynamics in less diverse communities.

\section{Science for development}

Mabisi is an important subject for science for development. It is a safe, popular, affordable, traditional fermented dairy product. Fermented products can provide much of the needed proteins, vitamins, and micronutrients to its consumers, including young children $(216,217)$. Besides, traditional fermented products are rooted in the local context and have high social and cultural value (218) (Figure 7.5).

Nutritional studies with Mabisi analysed by Optifood linear programming (219) showed that the addition of this fermented milk to the diet of children between 0 and 5 years old would increase the intake of essential vitamins to the daily recommended levels (220). Effects of the diet on the composition and metabolic profile of the microbial community in the human gut can be analysed using an artificial gut system, called SHIME (221). When Mabisi was included in the artificial diet of the SHIME in a MSc student project, the gut microbial communities showed an increase in short chain fatty acid (SCFA) production. Clinical studies showed a similar increase in SCFAs in stool samples of children that consumed Mabisi (222). As SCFAs are important metabolites in maintaining intestinal homeostasis (223), Mabisi is hypothesised to have a positive effect on human gut health.

In this thesis, science for development goes hand in hand with more fundamental studies in ecology and evolution. Findings in community dynamics and functional stability are important both for understanding ecological interactions as well as characteristics of the fermented product. Being able to predict community composition in a new environment and over time can be used to increase our understanding of ecoevolutionary dynamics in general along with outcomes of traditional fermentation with passive back-slopping. In other words, the headings above could have been different. Instead of "dissecting community functions", it could have said "starter culture development", and "community resilience" could have been called "product safety and stability". The research would have been the same, but the interpretation of the results would have differed.

During the PhD research conducted at the University of Zambia and Wageningen University, we have actively collaborated with local communities, NGOs, and institutional stakeholders in Zambia to design and carry out the research. 


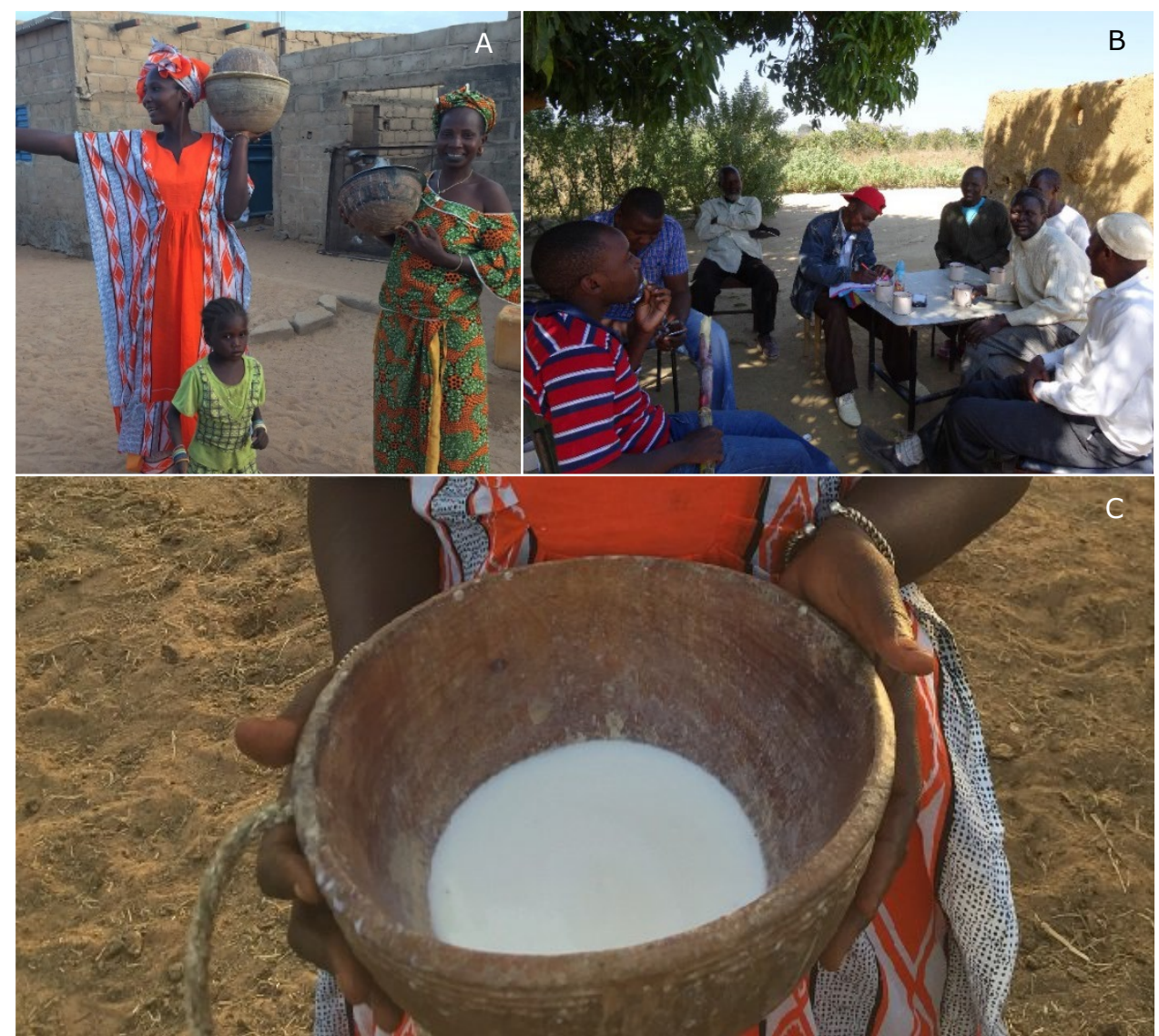

Figure 7.5 Producing and consuming your own fermented milk. In Zambia, Senegal and many other African countries the production and consumption of spontaneously fermented milk is embedded in the culture. A) Two women in Senegal hold the lahals that they use for Lait caille production. These lahals are often part of a wedding gift. B) Family and friends in Zambia join together to discuss business and pleasure over a cup of Mabisi. C) A lahal filled with milk will be covered and left to ferment to become Lait caillé. Pictures $A$ and $C$ by Megan Parker, Path.

The potential of Mabisi to alleviate poverty, food insecurity at household level, and malnutrition is currently underutilised due to a non-optimised food value chain (224). Optimizing the Mabisi value chain provides a promising means to overcome problems of hunger and malnutrition of both rural and urban low-resource consumers by promoting their nutrition security and health, while generating opportunities for women entrepreneurship. As a follow-up of the current project, I intend to:

(1) disseminate our scientific results and insights to both institutional stakeholders and local farmers who have been involved in the research;

(2) translate previous research findings to practical applications together with local producers and discuss options to promote women entrepreneurship. Optimisations in 
Mabisi production should be amendable for use by current small-scale producers, usually women, and can result in a definition of best practices;

(3) perform pilots of up-scaled and standardized Mabisi production, thereby implementing technological innovations, which lead to a higher quality product and a more efficient production process for local producers. A start will be made for the production of starter cultures that result in the nutritionally best and most appreciated Mabisi;

This three-month project is to be performed in collaboration with the Zambian partners. The focus will be on collaboration with two focal dairy cooperatives with which we have long-standing relationships. The main deliverable of this project will be an action plan on the possibilities of improving current practices and implementation of improvements.

\section{Concluding remarks}

The first aim of the thesis was to provide the first step towards the use of microbial communities of Mabisi as model system in studies towards eco-evolutionary dynamics. Now that the bacterial communities in Mabisi are known, including insights in diversity and changes in species composition over time, further studies towards the function and co-evolution of the species can be carried out.

The second aim was to provide ecological and evolutionary insights in Mabisi fermentations as part of a research for development project. We increased our knowledge of traditional spontaneous milk fermentation and gained knowledge about effects of common practices, such as active back-slopping and change of fermentation vessel, and uncommon practices, such as the addition of a probiotic starter culture. Furthermore, we gained understanding about the resilience of microbial communities against invasion of a pathogen. Improving production and increasing consumption of traditional, spontaneously fermented products will contribute towards tackling several sustainable development goals, in particular nutrition security, public health as well as woman entrepreneurship from a bottom-up approach. 


\section{References}

1. Schoustra SE. GCP-1 Zambian traditional fermented foods [Internet]. Food and Business Knowledge Platform. [cited 2019 Mar 5]. Available from: https://knowledge4food.net/researchproject/gcp1-zambian-traditionalfermented-foods/

2. Wolfe BE, Dutton RJJ. Fermented Foods as Experimentally Tractable Microbial Ecosystems. Cell. Elsevier Inc.; 2015;161(1):49-55.

3. Spus M. PhD thesis: Mixed culture engeneering for steering starter functionality. Wageningen University; 2016.

4. Fussmann GF, Loreau M, Abrams PA. Ecoevolutionary dynamics of communities and ecosystems. Funct Ecol. Wiley/Blackwell (10.1111); 2007 Jun 1;21(3):465-77.

5. Schoustra SE, Kasase C, Toarta C, Kassen $\mathrm{R}$, Poulain AJ. Microbial community structure of three traditional zambian fermented products: mabisi, chibwantu and munkoyo. PLoS One. 2013;8(5):e63948.

6. Kassen R. Experimental Evolution and the Nature of Biodiversity. Roberts and Company Publishers; 2014. 277 p.

7. Odunfa $S$ a. Review: African fermented foods: from art to science. MIRCEN J. 1988;4:259-73.

8. Moonga $H B$, Schoustra $S$, Linnemann $A$, Shindano J, Smid EJ. Influence of Production Methods on Communities of Lactic Acid Bacteria in Traditional Fermented Milk Mabisi. In: Short lectures, presented at the 12th Symposium on Lactic Acid Bacteria. 2017. p. 1.

9. Marco ML, Heeney D, Binda S, Cifelli CJ, Cotter PD, Foligné $B$, et al. Health benefits of fermented foods: microbiota and beyond. Curr Opin Biotechnol. 2017;44:94-102.

10. Ven $L$ van de. Consumer perspective on traditional Mabisi - Food \&amp; Business Knowledge Platform. Wageningen university and Research; 2018.

11. Spus M, Li M, Alexeeva S, WolkersRooijackers JCMCM, Zwietering MHH, Abee T, et al. Strain diversity and phage resistance in complex dairy starter cultures. J Dairy Sci. 2015;98(8):5173-82.

12. Gatti M, Bottari B, Lazzi C, Neviani E, Mucchetti G. Invited review: Microbial evolution in raw-milk, long-ripened cheeses produced using undefined natural whey starters. J Dairy Sci. 2014;97(2):573-91.

13. Nout MJR. Accelerated natural lactic fermentation of cereal-based formulas at reduced water activity. Int J Food Microbiol. $1992 ; 16(4): 313-22$.

14. Darwin CR. On the Origin of Species by means of natural selection, or the preservation of favoured races in the struggle for life. John Murray London; 1859.

15. Gibbons JG, Rinker DC. The genomics of microbial domestication in the fermented food environment. Curr Opin Genet Dev. Elsevier Current Trends; 2015 Dec 1;35:1-8.

16. Douglas GL, Klaenhammer TR. Genomic Evolution of Domesticated Microorganisms. Annu Rev Food Sci Technol. Annual Reviews ; 2010 Apr 4;1(1):397-414.

17. Bell T, Newman JJ a, Silverman BBW, Turner SLS, Lilley AAK. The contribution of species richness and composition to bacterial services. Nature. 2005;436(7054):1157-60.

18. Schreiber SJ, Vejdani M. Handling time promotes the coevolution of aggregation in predator-prey systems. Proc Biol Sci. 2006;273(1583):185-91. 
19. Pimentel D. Animal Population Regulation by the Genetic Feed-Back Mechanism. Am Nat. 1961;95(881):65-79.

20. Van Dyken JD, Müller MJI, MacK KML, Desai MM. Spatial population expansion promotes the evolution of cooperation in an experimental prisoner's dilemma. Curr Biol. 2013;23:919-23.

21. Oliveira NM, Niehus $R$, Foster $K R$. Evolutionary limits to cooperation in microbial communities. Proc Natl Acad Sci. 2014;111(50):201412673.

22. Sieuwerts $S$, Molenaar $D$, van Hijum $S$ a. FT, Beerthuyzen M, Stevens MJ a., Janssen PWM, et al. Mixed-Culture Transcriptome Analysis Reveals the Molecular Basis of Mixed-Culture Growth in Streptococcus thermophilus and Lactobacillus bulgaricus ${ }^{\dagger}$. Appl .... 2010;76(23):7775-84.

23. Hillesland KL, Stahl D a. Rapid evolution of stability and productivity at the origin of a microbial mutualism. Proc Natl Acad Sci U S A. 2010 Feb 2;107(5):2124-9.

24. Anonymous (British Ecological Society). Symposium on 'The ecology of closely allied species'. J Anim Ecol. 1944;13(2):176-7.

25. Gause GF, witt. Behavior of Mixed Populations and the Problem of Natural Selection. Am Nat. 1935;69(725):596.

26. Gause F. The Struggle for Existence. Vol. 7, The Yale journal of biology and medicine. Courier Corporation; 1934. 609 p.

27. Sieuwerts S, De Bok FAM, Hugenholtz J, Van Hylckama Vlieg JET. Unraveling microbial interactions in food fermentations: From classical to genomics approaches. Appl Environ Microbiol. 2008;74(16):4997-5007.

28. Kassen R, Rainey PB. The ecology and genetics of microbial diversity. Annu Rev Microbiol. 2004;58:207-31.

29. Meyer JR, Kassen R. The effects of competition and predation on diversification in a model adaptive radiation. Nature. 2007;446(7134):432-5.
30. Rainey PB, Buckling A, Kassen R, Travisano $M$. The emergence and maintenance of diversity: Insights from experimental bacterial populations. Vol. 15, Trends in Ecology and Evolution. 2000. p. 243-7.

31. Kassen R, Bell G. The ecology and genetics of fitness in Chlamydomonas. $X$. The relationship between genetic correlation and genetic distance. Evolution. 2000;54(2):425-32.

32. Jessup CM, Kassen $R$, Forde $S E$, Kerr $B$, Buckling $A$, Rainey $P B$, et al. Big questions, small worlds: Microbial model systems in ecology. Trends Ecol Evol. 2004;19(4):18997.

33. Goodnight $\mathrm{CJ}$. Experimental Studies of Community Evolution I: The Response to Selection at the Community Level. Evolution (N Y). 1990;44(6): 1614.

34. Wright CK. Ecological community integration increases with added trophic complexity. Ecol Complex. 2008;5(2):140-5.

35. Swenson W, Wilson DS, Elias R. Artificial ecosystem selection. Proc Natl Acad Sci. 2000;97(16):9110-4.

36. Clutton-Brock T, Pemberton J. Soay Sheep: Dynamics and selection in an island population. Cambridge: Cambridge University Press; 2004.

37. Grant PR, Grant BR, Smith JN, Abbott IJ, Abbott LK. Darwin's finches: population variation and natural selection. Proc Natl Acad Sci. 1976;73(1):257-61.

38. Lack D. Darwin's Finches. Cambridge University Press; 1947. 208 p.

39. Seehausen O. Process and pattern in cichlid radiations-inferences for understanding unusually high rates of evolutionary diversification. New Phytol. Blackwell Publishing Ltd; 2015 Jul;207(2):304-12.

40. Gould. Wonderful life: The Burgess shale and the nature of history. Trends Ecol Evol. 
W. W. Norton \& Co., New York and London; 1990 May 1;5(11):375-6.

41. Kawecki TJ, Lenski RE, Ebert D, Hollis $B$, Olivieri I, Whitlock MC. Experimental evolution. Trends Ecol Evol. 2012;27(10):547-60.

42. Bachmann H, Starrenburg MJC, Molenaar D, Hylckama Vlieg JET Van. Microbial domestication signatures of Lactococcus lactis can be reproduced by experimental evolution. Bioinformatics. 2011;1-35.

43. Celiker H, Gore J. Clustering in community structure across replicate ecosystems following a long-term bacterial evolution experiment. Nat Commun. 2014;5.

44. Venturelli OS, Carr AC, Fisher G, Hsu RH, Lau $R$, Bowen $B P$, et al. Deciphering microbial interactions in synthetic human gut microbiome communities. Mol Syst Biol. EMBO Press; 2018 Jun 21;14(6):e8157.

45. Mao J, Blanchard AE, Lu T. Slow and Steady Wins the Race: A Bacterial Exploitative Competition Strategy in Fluctuating Environments. ACS Synth Biol. American Chemical Society; 2015 Mar 20;4(3):240-8.

46. Hendry A. Eco-evolutionary dynamics. 2016.

47. Whittaker R, Fernández-Palacios J. Island biogeography: ecology, evolution, and conservation. Oxford University Press, Oxford; 2007. 401 p.

48. Wolfe BE, Dutton RJ. Fermented Foods as Experimentally Tractable Microbial Ecosystems. Cell. Elsevier Inc.; 2015;161(1):49-55.

49. Cocolin L, Alessandria V, Dolci P, Gorra R, Rantsiou K. Culture independent methods to assess the diversity and dynamics of microbiota during food fermentation. Int $\mathrm{J}$ Food Microbiol. Elsevier B.V.; 2013;167(1):29-43.

50. Sekwati-Monang B, Valcheva R, Gänzle MG. Microbial ecology of sorghum sourdoughs: Effect of substrate supply and phenolic compounds on composition of fermentation microbiota. Int J Food Microbiol. Elsevier B.V.; 2012 Oct 15;159(3):240-6.

51. Madoroba E, Steenkamp ET, Theron J, Scheirlinck I, Cloete TE, Huys G. Diversity and dynamics of bacterial populations during spontaneous sorghum fermentations used to produce ting, a South African food. Syst Appl Microbiol. Elsevier GmbH.; 2011;34(3):22734.

52. Smid EJ, Lacroix C. Microbe-microbe interactions in mixed culture food fermentations. Curr Opin Biotechnol. Elsevier Ltd; 2013;24(2):148-54.

53. De Roy K, Marzorati M, Van den Abbeele $P$, Van de Wiele T, Boon N. Synthetic microbial ecosystems: an exciting tool to understand and apply microbial communities. Environ Microbiol. 2014;16(6):1472-81.

54. Blasche S, Kim Y, Oliveira AP, Patil KR. Model Microbial Communities for Ecosystems Biology. Curr Opin Syst Biol. Elsevier Ltd; 2017;6:51-7.

55. Gravel D, Bell T, Barbera C, Bouvier $T$, Pommier $T$, Venail $P$, et al. Experimental niche evolution alters the strength of the diversity-productivity relationship. Nature. Nature Publishing Group; 2011;469(7328):89-92.

56. Lawrence $D$, Fiegna $F$, Behrends $V$, Bundy $J G$, Phillimore $A B$, Bell $T$, et al. Species interactions alter evolutionary responses to a novel environment. PLoS Biol. 2012;10(5).

57. Rivett DW, Bell T. Abundance determines the functional role of bacterial phylotypes in complex communities. Nat Microbiol. Nature Publishing Group; 2018 Jul 18;3(7):767-72.

58. Wolfe BE, Dutton RJ. Fermented Foods as Experimentally Tractable Microbial Ecosystems. Cell. Elsevier Inc.; 2015;161(1):49-55.

59. Atwood KC, Schneider LK, Ryan FJ. Periodic Selection in Escherichia Coli. Proc Natl Acad Sci. 1951;37(3):146-55. 
60. Dykhuizen DE, Hartl DL. Selection in Chemostats S ( $1_{-}$y [ Ks ( 1 ). Microbiol Rev. 1983;47(2):150-68.

61. Lenski RE, Rose MR, Simpson SC, Tadler SC. Long-Term Experimental Evolution in Escherichia coli. I. Adaptation and Divergence During 2,000 Generations. Am Nat. $1991 ; 138(6): 1315-41$.

62. Lenski RE. Experimental evolution and the dynamics of adaptation and genome evolution in microbial populations. ISME J. Nature Publishing Group; 2017;11(10):2181-94.

63. Elena SSF, Lenski RER. Evolution experiments with microorganisms: The dynamics and genetic bases of adaptation. Nat Rev Genet. 2003;4(6):457-69.

64. de Visser JAGM, Krug J. Empirical fitness landscapes and the predictability of evolution. Nat Rev Genet. Nature Publishing Group; 2014;15(7):480-90.

65. Lenski RE, Ofria C, Collier TC, Adami C. Genome complexity, robustness and genetic interactions in digital organisms. Nature. 1999;400(6745):661-4.

66. Lenski RE. Convergence and Divergence in a Long-Term Experiment with Bacteria. Agrawal A, editor. Am Nat. 2017 Mar;190(S1):S57-68.

67. Dettman JR, Rodrigue $N$, Melnyk $A H$, Wong A, Bailey SF, Kassen R. Evolutionary insight from whole-genome sequencing of experimentally evolved microbes. Mol Ecol. 2012 May;21(9):2058-77.

68. Wittebolle $L$, Marzorati $M$, Clement $L$, Balloi A, Daffonchio D, Heylen $K$, et al. Initial community evenness favours functionality under selective stress. Nature. Nature Publishing Group; 2009;458(7238):623-6.

69. De Roy K, Marzorati M, Negroni A, Thas $O$, Balloi A, Fava $F$, et al. Environmental conditions and community evenness determine the outcome of biological invasion. Nat Commun. 2013 Jan 22;4:1383.
70. Großkopf T, Soyer OS. Synthetic microbial communities. Curr Opin Microbiol. Elsevier Ltd; 2014;18(1):72-7.

71. Friedman J, Higgins LM, Gore J. Community structure follows simple assembly rules in microbial microcosms. Nat Ecol Evol. Macmillan Publishers Limited, part of Springer Nature.; 2017;1(5):1-7.

72. Fredrickson JK. Ecological communities by design. Science (80- ). 2015;348(6242):1425-7.

73. Røder $\mathrm{HL}$, Sørensen SJ, Burmølle $M$. Studying Bacterial Multispecies Biofilms: Where to Start? Trends Microbiol. 2016 Jun;24(6):503-13.

74. Cairns J, Jokela R, Hultman J, Tamminen M, Virta M, Hiltunen T. Construction and characterization of synthetic bacterial community for experimental ecology and evolution. Front Genet. Frontiers Media SA; 2018;9(AUG):312.

75. Yu Z, Krause SMB, Beck DAC, Chistoserdova L. A synthetic ecology perspective: How well does behavior of model organisms in the laboratory predict microbial activities in natural habitats? Front Microbiol. 2016;7(JUN).

76. Fiegna F, Moreno-Letelier A, Bell $T$, Barraclough TG. Evolution of species interactions determines microbial community productivity in new environments. ISME J. Nature Publishing Group; 2015;9(5):123545.

77. Erkus O, De Jager VCL, Spus M, Van AlenBoerrigter IJ, Van Rijswijck IMH, Hazelwood $\mathrm{L}$, et al. Multifactorial diversity sustains microbial community stability. ISME J. Nature Publishing Group; 2013;7(11):2126-36.

78. Bessmeltseva M, Viiard E, Simm J, Paalme T, Sarand I. Evolution of bacterial consortia in spontaneously started rye sourdoughs during two months of daily propagation. PLoS One. Public Library of Science; 2014 Jan 18;9(4):e95449. 
79. Smid EJ, Erkus O, Spus M, WolkersRooijackers JCM, Alexeeva S, Kleerebezem M. Functional implications of the microbial community structure of undefined mesophilic starter cultures. Microb Cell Fact. 2014;13(1).

80. Moonga HB, Schoustra SE, Linnemann AR, Kuntashula E, Shindano J, Smid EJ. The art of Mabisi production: a traditional fermented milk. PLoS One. 2019; In press.

81. Gadaga TH, Mutukumira a. N, Narvhus J a., Feresu SB. A review of traditional fermented foods and beverages of Zimbabwe. Int J Food Microbiol. 1999;53:111.

82. Ravyts F, Vuyst L De, Leroy F. Bacterial diversity and functionalities in food fermentations. Eng Life Sci. 2012;12(4):356-67.

83. Franz CM a. $\mathrm{P}$, Huch $\mathrm{M}$, Mathara JM, Abriouel H, Benomar N, Reid G, et al. African Fermented Foods and Probiotics. Int J Food Microbiol. Elsevier B.V.; 2014 Aug;190:8496.

84. Tamang JP, Watanabe K, Holzapfel WH. Review: Diversity of microorganisms in global fermented foods and beverages. Front Microbiol. 2016;7(377):1-28.

85. Teusink B, Smid EJ. Modelling strategies for the industrial exploitation of lactic acid bacteria. Nat Rev Microbiol. 2006;4(1):4656.

86. Teusink B, Wiersma A, Molenaar D, Francke C, De Vos WM, Siezen RJ, et al. Analysis of growth of Lactobacillus plantarum WCFS1 on a complex medium using a genome-scale metabolic model. J Biol Chem. 2006;281(52):40041-8.

87. Gänzle MG. Lactic metabolism revisited: Metabolism of lactic acid bacteria in food fermentations and food spoilage. Curr Opin Food Sci. 2015;2:106-17.

88. Carbonetto BB, Ramsayer J, Nidelet $T$, Legrand J, Sicard D. Bakery yeasts, a new model for studies in ecology and evolution. Yeast. 2018;35(March):1-13.

89. Fiegna F, Scheuerl T, Moreno-Letelier A, Bell T, Barraclough TG. Saturating effects of species diversity on life-history evolution in bacteria. Proc Biol Sci. 2015;282(1815):20151794-.

90. Székely AJ, Langenheder S. The importance of species sorting differs between habitat generalists and specialists in bacterial communities. FEMS Microbiol Ecol. 2014 Jan;87(1):102-12.

91. Przeworski M, Coop G, Wall JD. The Signature of Positive Selection on Standing Genetic Variation. Evolution (N Y). John Wiley \& Sons, Ltd (10.1111); 2006 Nov $1 ; 59(11): 2312$.

92. Teotónio H, Chelo IM, Bradić M, Rose MR, Long AD. Experimental evolution reveals natural selection on standing genetic variation. Nat Genet. Nature Publishing Group; 2009 Feb 11;41(2):251-7.

93. McPeek MA. Evolutionary community ecology. 2017. 313 p.

94. Stearns S. The Emergence of Evolutionary and Community Ecology as Experimental Sciences. ResearchgateNet. $1982 ; 25(4): 621-48$.

95. Johnson MTJJ, Stinchcombe JR. An emerging synthesis between community ecology and evolutionary biology. pdfs.semanticscholar.org. 2007;22(5):2507 .

96. Wilcox TMT, Wilcox TMT, Schwartz MKMM, Lowe WHWW. Evolutionary Community Ecology: Time to Think Outside the (Taxonomic) Box. researchgate.net. 2018;33(4):240-50.

97. McPeek MA. The Ecological Dynamics of Natural Selection: Traits and the Coevolution of Community Structure. Am Nat. 2017;189(5): E91-117.

98. McCann KS. The diversity-stability debate. Nature. 2000;405(6783):228-33. 
99. Awasthi A, Singh M, Soni SK, Singh R, Kalra A. Biodiversity acts as insurance of productivity of bacterial communities under abiotic perturbations. Isme J. 2014;8(12):2445-52.

100. Mallon CA, Van Elsas JD, Salles JF. Microbial invasions: The process, patterns, and mechanisms. Trends Microbiol. Elsevier Ltd; 2015 Oct $11 ; 23(11)$ :719-29.

101. Thingstad TF, Lignell R. Theoretical models for the control of bacterial growth rate, abundance, diversity and carbon demand. Aquat Microb Ecol. 1997;13:19-27.

102. Feldgarden M, Stoebel DM, Brisson D, Dykhuizen DE. Size doesn't matter: Microbial selection experiments address ecological phenomena. Ecology. 2003;84(7):1679-87.

103. Koskella B, Lively CM. Evidence for negative frequency-dependent selection during experimental coevolution of a freshwater snail and a sterilizing trematode. Evolution (N Y). 2009 Sep;63(9):2213-21.

104. Hardin G. The Competitive Exclusion Principle. Science (80- ). 1960;131(3409):1292-7.

105. Gause GF. Experimental Analysis of Vito Volterra'S Mathematical Theory of the Struggle for Existence. Science (80- ). 1934;79(2036): 16-7.

106. Hutchinson GE. Concluding Remarks. Cold Spring Harb Symp Quant Biol. 1957;22(0):415-27.

107. Ashby B, Watkins E, Lourenço J, Gupta $\mathrm{S}$, Foster KR. Competing species leave many potential niches unfilled. Nat Ecol Evol. 2017;1(10):1495-501.

108. May RM. On the theory of niche overlap. Theor Popul Biol. 1974;5(3):297-332.

109. Pacala SW, Roughgarden J. The evolution of resource partitioning in a multidimensional resource space. Theor Popul Biol. 1982;22(1):127-45.
110. Stecher B, Chaffron $S$, Käppeli R, Hapfelmeier S, Freedrich S, Weber TC, et al. Like will to like: Abundances of closely related species can predict susceptibility to intestinal colonization by pathogenic and commensal bacteria. PLoS Pathog. 2010;6(1).

111. Stecher B, Berry D, Loy A. Colonization resistance and microbial ecophysiology: Using gnotobiotic mouse models and singlecell technology to explore the intestinal jungle. FEMS Microbiol Rev. 2013;37(5):793-829.

112. Grant PR. Convergent and divergent character displacement. Biol J Linn Soc. 1972;4(1):39-68.

113. Rainey PB, Travisano M. Adaptive radiation in a heterogeneous environment. Nature. 1998;394:69-72.

114. Reznick DN, Bryant MJ, Bashey F. r-and $\mathrm{K}$-selection revisited: the role of population regulation in life-history evolution. Ecology. 2002;83(6):1509-20.

115. Fitzsimmons JM, Schoustra SE, Kerr JT, Kassen R. Population consequences of mutational events: Effects of antibiotic resistance on the $\mathrm{r} / \mathrm{K}$ trade-off. Evol Ecol. 2010;24(1):227-36.

116. van Mastrigt O, Abee T, Lillevang SK, Smid EJ. Quantitative physiology and aroma formation of a dairy Lactococcus lactis at near-zero growth rates. Food Microbiol. Elsevier Ltd; 2018;73:216-26.

117. Rillig MC, Antonovics J, Caruso $T$, Lehmann A, Powell JR, Veresoglou SD, et al. Interchange of entire communities: Microbial community coalescence. Trends Ecol Evol. Elsevier Ltd; 2015;30(8):470-6.

118. Tikhonov M. Community-level cohesion without cooperation. Elife. 2016;5:1-12.

119. Sierocinski $\mathrm{P}$, Milferstedt $\mathrm{K}$, Bayer $\mathrm{F}$, Großkopf T, Alston M, Bastkowski S, et al. A Single Community Dominates Structure and Function of a Mixture of Multiple Methanogenic Communities. Curr Biol. 2017;27(21):3390-5. 
120. Kort R, Sybesma W. Probiotics for every body. Trends Biotechnol. 2012;30(12):6135.

121. Van den Abbeele $P$, Grootaert $C$, Marzorati M, Possemiers S, Verstraete W, Gérard $P$, et al. Microbial community development in a dynamic gut model is reproducible, colon region specific, and selective for Bacteroidetes and Clostridium cluster IX. Appl Environ Microbiol. American Society for Microbiology (ASM); 2010 Aug;76(15):5237-46.

122. Dallinger W. The President's address. J Microsc. 1887;

123. Rozen DE, Lenski RE. Long-Term Experimental Evolution in Escherichia coli . VIII. Dynamics of a Balanced Polymorphism. Am Nat. 2000;155(1):24-35.

124. Lenski RE, Travisano M. Dynamics of adaptation and diversification: a 10,000generation experiment with bacterial populations. Proc Natl Acad Sci. 1994;91(15):6808-14.

125. Deatherage $D E$, Kepner JL, Bennett $A F$, Lenski RE, Barrick JE. Specificity of genome evolution in experimental populations of Escherichia coli evolved at different temperatures. Proc Natl Acad Sci U S A. 2017 Mar 7;114(10):E1904-12.

126. Sachs JL, Hollowell AC. The origins of cooperative bacterial communities. MBio. 2012;3(3):1-3.

127. Cremer J, Melbinger A, Frey E. Growth dynamics and the evolution of cooperation in microbial populations. Sci Rep. 2012;2:1-6.

128. Zabat M, Sano W, Wurster J, Cabral D, Belenky P. Microbial Community Analysis of Sauerkraut Fermentation Reveals a Stable and Rapidly Established Community. Foods. $2018 ; 7(5): 77$.

129. Audrain B, Farag MA, Ryu CM, Ghigo JM. Role of bacterial volatile compounds in bacterial biology. FEMS Microbiol Rev. Oxford University Press; 2015 Mar 1;39(2):222-33.
130. The Nagoya Protocol on Access and Benefit-sharing [Internet]. [cited 2019 Mar 5]. Available from: https://www.cbd.int/abs/

131. Sybesma W, Molenaar D, van IJcken W, Venema K, Kort R. Genome Instability in Lactobacillus rhamnosus GG. Appl Environ Microbiol. 2013;79(7):2233-9.

132. Tomlinson J, Boonham N, Hughes $\mathrm{K}$, Griffin R, Barker I. On-Site DNA Extraction and Real-Time PCR for Detection of Phytophthora ramorum in the Field. Appl Environ Microbiol. 2005;71(11):6702-10.

133. Swinfield CE, Graham EAM, Nuttall $D$, Maguire S, Kemp A, Rutty GN. The use of DNA stabilizing solution to enable room temperature storage and transportation of buccal and trace sample swabs. Forensic Sci Int Genet Suppl Ser. 2009;2(1):183-4.

134. Terada ASSD, Silva LAF Da, Galo R, Azevedo A De, Gerlach RF, Silva RHA Da. The use of a DNA stabilizer in human dental tissues stored under different temperature conditions and time intervals. J Appl Oral Sci. 2014;22(4):331-5.

135. Mitchell KR, Takacs-Vesbach CD. A comparison of methods for total community DNA preservation and extraction from various thermal environments. J Ind Microbiol Biotechnol. 2008 Oct 17;35(10):1139-47.

136. Fomovskaia G, Smith MA, Davis JC, Jones K. International Patent: Fta-coated media for use as a molecular diagnostic tool. 2000. p. 51.

137. Menassa N, Bosshard PPP, Kaufmann C, Grimm C, Auffarth GU, Thiel MA. Rapid Detection of Fungal Keratitis with DNAStabilizing FTA Filter Paper. Investig Opthalmology Vis Sci. 2010;51(4):1905-10.

138. Burgoyne LA. United States Patent: Solid medium and method for DNA storage. 1996. p. 8.

139. Lore T a., Mbugua SK, Wangoh J. Enumeration and identification of microflora in suusac, a Kenyan traditional fermented 
camel milk product. LWT - Food Sci Technol. 2005;38(2):125-30.

140. Ercolini D, Moschetti G, Blaiotta G, Coppola $S$. The potential of a polyphasic PCRdGGE approach in evaluating microbial diversity of natural whey cultures for waterbuffalo Mozzarella cheese production: bias of culture-dependent and culture-independent analyses. Syst Appl Microbiol. $2001 ; 24(4): 610-7$.

141. Caporaso JG, Fierer N, Peña AG, Goodrich JK, Gordon JI, Huttley GA, et al. QIIME allows analysis of high-throughput community sequencing data. Nat Methods. $2010 ; 7(5): 335-6$.

142. Bik HM, Maritz JM, Luong $A$, Shin $H$, Dominguez-Bello MG, Carlton JM. Microbial Community Patterns Associated with Automated Teller Machine Keypads in New York City. mSphere. 2016;1(6):e00226-16.

143. Rajendram D, Ayenza R, Holder FM, Moran B, Long $T$, Shah HN. Long-term storage and safe retrieval of DNA from microorganisms for molecular analysis using FTA matrix cards. J Microbiol Methods. 2006;67(3):582-92.

144. Gray M a., Pratte ZA, Kellogg CA. Comparison of DNA preservation methods for environmental bacterial community samples. FEMS Microbiol Ecol. 2013 Feb;83(2):46877.

145. Smith L, Burgoyne L. Collecting, archiving and processing DNA from wildlife

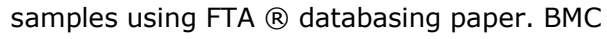
Ecol. 2004;4(1):4.

146. Altschul SF, Gish W, Miller W, Myers EW, Lipmanl DJ. Basic Local Alignment Search Tool. J Mol Biol. 1990;215:403-10.

147. Dertli E, Çon AH. Microbial diversity of traditional kefir grains and their role on kefir aroma. LWT - Food Sci Technol. 2017;85:151-7.

148. Philippot L, Hallin S, Sá Nchez S, Olson B. Finding the missing link between diversity and activity using denitrifying bacteria as a model functional community. Curr Opin Microbiol. 2005;8(Ecology and industrial microbiology):234-9.

149. Randazzo CL, Vaughan EE, Caggia C. Artisanal and experimental Pecorino Siciliano cheese: Microbial dynamics during manufacture assessed by culturing and PCRDGGE analyses. Int J Food Microbiol. 2006;109(1-2):1-8.

150. van Rijswijck IMH, Wolkers Rooijackers JCM, Abee T, Smid EJ. Performance of non-conventional yeasts in co-culture with brewers' yeast for steering ethanol and aroma production. Microb Biotechnol. 2017;10(6):1591-602.

151. Fernández De Palencia $P$, De La Plaza $M$, Amárita $F$, Requena $T$, Peláez C. Diversity of amino acid converting enzymes in wild lactic acid bacteria. Enzyme Microb Technol. 2006;38(1-2):88-93.

152. Groenenboom $A E$, Parker ME, Vries $A$ de, Groot S de, Zobrist S, Mansen K, et al. Bacterial community dynamics in Lait caillé, a traditional product of spontaneous fermentation from Senegal. PLoS ONE. 2019; 14(5): e0215658.

153. Groenenboom AE, Smid EJ, Schoustra SE. Robust sampling and preservation of DNA for microbial community profiling in field experiments. BMC Research Notes. 2019; 12:159.

154. Martin M. Cutadapt removes adapter sequences from high-throughput sequencing reads. EMBnet.journal. 2011;17(1):10.

155. Haas R, Clemente B, Edgar RC, Haas BJ, Clemente JC, Quince $C$, et al. UCHIME improves sensitivity and speed of chimera detection.

Bioinformatics.

2011;27(16):2194-200.

156. Hutcheson K. A Test for Comparing Diversities Based on the Shannon Formula. J Theor Biol. 1970 Oct;29(1):151-4.

157. Whiting RC, Cygnarowicz-Provost M. A quantitative model for bacterial growth and decline. Food Microbiol. 1992;9(4):269-77. 
158. Jiang L, Morin PJ. Temperature fluctuation facilitates coexistence of competing species in experimental microbial communities. J Anim Ecol. John Wiley \& Sons, Ltd (10.1111); 2007 Jul 1;76(4):660-8.

159. Roxburgh SH, Shea K, Wilson JB. The intermediate disturbance hypothesis: Patch dynamics and mechanisms of species coexistence. Ecology. John Wiley \& Sons, Ltd; 2004 Feb 1;85(2):359-71.

160. Mpofu A, Linnemann AR, Nout MJR, Zwietering $\mathrm{MH}$, Smid EJ, den Besten HMW. Inactivation of bacterial pathogens in yoba mutandabota, a dairy product fermented with the probiotic Lactobacillus rhamnosus yoba. Int J Food Microbiol. 2016;217:42-8.

161. Lucey JA. Cultured dairy products: an overview of their gelation and texture properties. Int J Dairy Technol. John Wiley \& Sons, Ltd (10.1111); 2004 May 1;57(23):77-84.

162. Smid EJ, Kleerebezem M. Production of Aroma Compounds in Lactic Fermentations. Annu Rev Food Sci Technol. 2014;5(1):31326.

163. Stecher B, Berry D, Loy A. Colonization resistance and microbial ecophysiology: Using gnotobiotic mouse models and singlecell technology to explore the intestinal jungle. FEMS Microbiol Rev. 2013 Sep;37(5):793-829.

164. Freilich S, Zarecki R, Eilam O, Segal ES, Henry CS, Kupiec M, et al. Competitive and cooperative metabolic interactions in bacterial communities. Nat Commun. 2011;2(1):587-9.

165. Zaret TM, Rand AS. Competition in Tropical Stream Fishes: Support for the Competitive Exclusion Principle. Ecology. John Wiley \& Sons, Ltd; 2006 Mar $1 ; 52(2): 336-42$.

166. Langenheder S, Székely AJ. Species sorting and neutral processes are both important during the initial assembly of bacterial communities. ISME J. 2011;5(7):1086-94.
167. Chase J, Zare M, Knight R, Sonderegger DL, Siegel J, Kelley ST, et al. Geography and Location Are the Primary Drivers of Office Microbiome Composition. mSystems. $2016 ; 1(2)$.

168. McFadden D. Conditional logit analysis of qualitative choice behaviour. Frontiers in econometrics. In: Frontiers in Economics, P. Zarembka, eds. New York: Academic Press.; 1974. 105-142 p.

169. Travisano M, Mongold J, Bennett A, Lenski R. Experimental tests of the roles of adaptation, chance, and history in evolution. Science(Washington) 1995.

170. Blanton L V., Charbonneau MR, Salih $T$, Barratt MJ, Venkatesh S, Ilkaveya O, et al. Gut bacteria that prevent growth impairments transmitted by microbiota from malnourished children. Science (80- ). 2016;351(6275).

171. Gning MC. Trade, political influence and liberalization: situating the poor in the political economy of livestock in Senegal. PPLPI Work Pap - Pro-Poor Livest Policy Initiat FAO. $2004 ;(8): 38$.

172. Parker M, Zobrist S, Donahue C, Edick C, Mansen K, Hassan Zade Nadjari M, et al. Naturally Fermented Milk From Northern Senegal: Bacterial Community Composition and Probiotic Enrichment With Lactobacillus rhamnosus. Front Microbiol. 2018 Sep 21;9.

173. Zobrist S, Kalra N, Pelto G, Wittenbrink $B$, Milani P, Diallo AM, et al. Results of Applying Cultural Domain Analysis Techniques and Implications for the Design of Complementary Feeding Interventions in Northern Senegal. Food Nutr Bull. SAGE PublicationsSage CA: Los Angeles, CA; 2017 Dec 24;38(4):512-27.

174. Zobrist S, Kalra N, Pelto G, Wittenbrink $B$, Milani $P$, Diallo AM, et al. Using cognitive mapping to understand Senegalese infant and young child feeding decisions. Matern Child Nutr. Wiley/Blackwell (10.1111); 2018 Apr $1 ; 14(2): \mathrm{e} 12542$. 
175. Kebede A, Viljoen BC, Gadaga TH, Narvhus JA, Lourens-Hattingh $A$. The effect of container type on the growth of yeast and lactic acid bacteria during production of Sethemi, South African spontaneously fermented milk. Food Res Int. 2007;40(1):33-8.

176. Mathara JM, Schillinger U, Guigas C, Franz C, Kutima PM, Mbugua SK, et al. Functional characteristics of Lactobacillus spp. from traditional Maasai fermented milk products in Kenya. Int J Food Microbiol. 2008; 126(1-2):57-64.

177. Kort R, Westerik N, Mariela Serrano L, Douillard FP, Gottstein W, Mukisa IM, et al. A novel consortium of Lactobacillus rhamnosus and Streptococcus thermophilus for increased access to functional fermented foods. Microb Cell Fact. BioMed Central; 2015;14(1).

178. Haegeman B, Hamelin J, Moriarty J, Neal P, Dushoff J, Weitz JS, et al. Robust estimation of microbial diversity in theory and in practice. ISME J. Nature Publishing Group; 2013 Jun;7(6):1092-101.

179. Fortina MG, Nicastro G, Carminati D, Neviani E, Manachini PL. Lactobacillus helveticus heterogeneity in natural cheese starters: The diversity in phenotypic characteristics. J Appl Microbiol. 1998 Jan;84(1):72-80.

180. Broadbent JR, Cai H, Larsen RL, Hughes JE, Welker DL, De Carvalho VG, et al. Genetic diversity in proteolytic enzymes and amino acid metabolism among Lactobacillus helveticus strains. J Dairy Sci. Elsevier; 2011 Sep 1;94(9):4313-28.

181. Broadbent JR, McMahon DJ, Welker DL, Oberg CJ, Moineau S. Biochemistry, Genetics, and Applications of Exopolysaccharide Production in Streptococcus thermophilus: A Review. J Dairy Sci. 2003;86(2):407-23.

182. Iyer $\mathrm{R}$, Tomar SK, Uma Maheswari $\mathrm{T}$, Singh R. Streptococcus thermophilus strains: Multifunctional lactic acid bacteria. Int Dairy J. Elsevier Ltd; 2010;20(3):133-41.
183. Dabboussi $F$, Hamze $M$, Elomari $M$, Verhille $S$, Baida $S$, Izarb $D$, et al. Pseudornonas libanensis sp. nov., a new species isolated from Lebanese spring waters. Int J Syst Bacteriol. 1999;49(1 999): 1091-101.

184. Müller HE, Brenner DJ, Fanning GR, Grimont P a, Kämpfer P. Emended description of Buttiauxella agrestis with recognition of six new species of Buttiauxella and two new species of Kluyvera: Buttiauxella ferragutiae sp. nov., Buttiauxella gaviniae sp. nov., Buttiauxella brennerae sp. nov., Buttiauxella izardii sp. Int J Syst Bacteriol. 1996 Jan $1 ; 46(1): 50-63$.

185. Shaw S, Keddie RM. A numerical taxonomic study of the genus Kurthia with a revised description of Kurthia zopfii and a description of Kurthia gibsonii sp. nov. Syst Appl Microbiol. Gustav Fischer Verlag, Stuttgart/New York; 1983 Apr 1;4(2):25376.

186. Zanni E, Schifano E, Motta S, Sciubba F, Palleschi C, Mauri P, et al. Combination of metabolomic and proteomic analysis revealed different features among Lactobacillus delbrueckii subspecies bulgaricus and lactis strains while in vivo testing in the model organism Caenorhabditis elegans highlighted probiotic properties. Front Microbiol. 2017 Jun 28;8(JUN).

187. Guglielmotti DM, Marcó MB, Golowczyc M, Reinheimer JA, Quiberoni A del L. Probiotic potential of Lactobacillus delbrueckii strains and their phage resistant mutants. Int Dairy J. $2007 ; 17(8): 916-25$.

188. Nout MJR. Food Technologies: Fermentation. In: Encyclopedia of Food Safety. 2014. p. i.

189. Cleenwerck I, De Vos P. Polyphasic taxonomy of acetic acid bacteria: An overview of the currently applied methodology. Int J Food Microbiol. 2008; 125(1):2-14.

190. D'Souza G, Kost C. Experimental Evolution of Metabolic Dependency in 
Bacteria

PLOS

Genet.

2016;12(11):e1006364.

191. Kassen R. Toward a general theory of adaptive radiation: Insights from microbial experimental evolution. Ann N Y Acad Sci. Wiley/Blackwell (10.1111); 2009 Jun $1 ; 1168(1): 3-22$.

192. Steenackers HPH, Parijs I, Foster KRK, Vanderleyden J. Experimental evolution in biofilm populations. Banin E, editor. FEMS Microbiol Rev. 2016 May;40(3):373-97.

193. Shade A. Diversity is the question, not the answer. ISME J. 2017;11(1):1-6.

194. Mpofu A, Linnemann AR, Sybesma W, Kort R, Nout MJR, Smid EJ. Development of a locally sustainable functional food based on mutandabota, a traditional food in southern Africa. J Dairy Sci. 2014;97(5):2591-9.

195. Di Stefano E, White J, Seney S, Hekmat $S$, McDowell $T$, Sumarah $M$, et al. A novel millet-based probiotic fermented food for the developing world. Nutrients. 2017;9(5).

196. Adam E, Groenenboom AE, Kurm VK, Rajewska M, Schmidt RL, Tyc O, et al. Controlling the microbiome: microhabitat adjustments for successful biocontrol strategies in soil and human gut: Opinion article. Front Microbiol. 2016; in press(JUL).

197. Bolnick DI, Amarasekare P, Araújo MS, Bürger R, Levine JM, Novak $M$, et al. Why intraspecific trait variation matters in community ecology. Trends Ecol Evol. 2011 Apr;26(4):183-92.

198. Basu S, Chatterjee M, Ganguly S, Chandra PK. Effect of Lactobacillus rhamnosus GG in Persistent Diarrhea in Indian Children. J Clin Gastroenterol. 2007 Sep;41(8):756-60.

199. Erkuş O. Community Dynamics of Complex Starter Cultures for Gouda-type Cheeses and its Functional Consequences. edepot.wur.nl. 2014;

200. Klijn N, Weerkamp AH, De Vos WM. Identification of mesophilic lactic acid bacteria by using polymerase chain reactionamplified variable regions of 16S rRNA and specific DNA probes. Appl Environ Microbiol. 1991;57(11):3390-3.

201. Andrighetto C, Dea P De, Lombardi A, Neviani E, Rosetti L, Giraffa G. Molecular identification and cluster analysis of homofermentative thermophilic lactobacilli isolated from dairy products. Res mMcrobiology. 1998;149(9):631-43.

202. Arumugam M, Raes J, Pelletier E, Le Paslier D, Yamada T, Mende DR, et al. Enterotypes of the human gut microbiome. Nature. Nature Publishing Group; 2011 May $12 ; 473(7346): 174-80$

203. Chandra Raj K, Ingram L, MaupinFurlow J. Pyruvate decarboxylase: a key enzyme for the oxidative metabolism of lactic acid by Acetobacter pasteurianus. Arch Microbiol. Springer-Verlag; 2001 Dec $1 ; 176(6): 443-51$.

204. Coyte KZ, Schluter J, Foster KR. The ecology of the microbiome: Networks, competition, and stability. Science (80- ). 2015;350(6261):663-6.

205. Foster KR, Bell T. Competition, not cooperation, dominates interactions among culturable microbial species. Curr Biol. 2012;22(19): 1845-50.

206. Gobbetti M, Di Cagno R. The Behavior in Foods. In Springer, Boston, MA; 2012. p. 3960.

207. Blasche S, Kim Y, Mars R, Kafkia E, Maansson M, Machado D, et al. Emergence of stable coexistence in a complex microbial community through metabolic cooperation and spatio-temporal niche partitioning. bioRxiv. Cold Spring Harbor Laboratory; 2019 Feb 5;541870.

208. Dekkers BL. Creation of fibrous plant protein foods. Wageningen University and Research; 2018.

209. Collins S, Gardner A. Integrating physiological, ecological and evolutionary 
change: A Price equation approach. Ecol Lett. 2009 Aug;12(8):744-57.

210. Bairey E, Kelsic ED, Kishony R. Highorder species interactions shape ecosystem diversity. Nat Publ Gr. Nature Publishing Group; 2016;7:1-7.

211. Hutchison CA, Chuang R-Y, Noskov VN, Assad-Garcia N, Deerinck TJ, Ellisman MH, et al. Design and synthesis of a minimal bacterial genome. Science (80- ). 2016;351(6280).

212. Eisenhauer N, Schulz W, Scheu S, Jousset $A$. Niche dimensionality links biodiversity and invasibility of microbial communities. Pfrender M, editor. Funct Ecol. 2013 Feb $10 ; 27(1): 282-8$.

213. Bengtsson J. Which species? What kind of diversity? Which ecosystem function? Some problems in studies of relations between biodiversity and ecosystem function. Appl Soil Ecol. 1998;10:191-9.

214. Sorek R, Cossart P. Prokaryotic transcriptomics: A new view on regulation, physiology and pathogenicity. Nat Rev Genet. Nature Publishing Group; 2010 Jan 24;11(1):9-16.

215. van Rijswijck IMH. Co-cultivation of non-convential yeast with Saccharomyces cerevisiae to increase the aroma complexity of fermented beverages. 2017.

216. Burgess CM, Smid EJ, van Sinderen D. Bacterial vitamin B2, B11 and B12 overproduction: An overview. Int J Food Microbiol. Elsevier B.V.; 2009 Jul 31;133(12):1-7.

217. Smid EJ, Hugenholtz J. Functional Genomics for Food Fermentation Processes. Annu Rev Food Sci Technol - (new 2010). 2010;1(1):497-519.

218. Barrett $C B$, Reardon $T$, Webb $P$. Nonfarm income diversification and household livelihood strategies in rural Africa: Concepts, dynamics, and policy implications. Food Policy. 2001;26(4):31531.
219. Talsma EF, Borgonjen-van den Berg KJ, Melse-Boonstra A, Mayer E V, Verhoef $H$, Demir AY, et al. The potential contribution of yellow cassava to dietary nutrient adequacy of primary-school children in Eastern Kenya; the use of linear programming. Public Health Nutr. Cambridge University Press; 2018 Feb 2;21(02):365-76.

220. Chileshe J, Schoustra S, Talsma EF, Borgonjen-van den Berg $\mathrm{KJ}$, Handema R, Zwaan B, et al. Cereal and milk based fermented foods to improving local diets: linear programming for 1-5 years old children in Central province in Zambia show potential. 2019; Submitted.

221. Molly K, Woestyne M Vande, Smet I De, Verstraete $W$. Validation of the Simulator of the Human Intestinal Microbial Ecosystem (SHIME) Reactor Using Microorganismassociated Activities. Microb Ecol Health Dis. 1994 Jan 11;7(4):191-200.

222. Chileshe J, Marinda $P$, Hamaimbo $B$, Sikalima J, Handema R, Zwaan B, et al. Mabisi intake has a positive effect on gut microbial composition and short chain fatty acids (SCFAs) in children 6-24 months old in Mkushi and Namwala of Zambia. 2019; In prepara.

223. Parada Venegas $D$, De La Fuente $M K$, Landskron G, González M-J, Quera R, Dijkstra G, et al. Short Chain Fatty Acids (SCFAs)mediated gut epithelial and immune regulation and its relevance for Inflammatory Bowel Diseases. Front Immunol. Frontiers; 2019;10:277.

224. Trichopoulou A, Soukara S, Vasilopoulou E. Traditional foods: a science and society perspective. Trends Food Sci Technol. 2007;18(8):420-7. 


\section{Summary}

Traditional fermented products are an important part of the diet in many African countries. These products can provide much of the needed proteins, vitamins and other micro-nutrients to its consumers, which include young children. Also, traditional fermented products are rooted in the local context and have high social and cultural value. Increasing our understanding of these spontaneously fermented products can be used to increase the value chain of these products in order to increase economic stability of producers as well as nutritional intake in rural and urban areas.

Most traditional fermented products are produced by spontaneous fermentation, meaning that there is no starter culture added to initiate fermentation. As a consequence these products contain a diverse microbial community, which are responsible for the characteristics of the product. Often a back-slopping method is used, where a portion of previously fermented product inoculates fresh raw material. Unknowingly, producers of these products have domesticated these bacterial communities and allowed the species to co-evolve within their community.

In this way, these bacterial communities form a model system for ecological and evolutionary research. Historically, experimental evolution was mainly performed with single organisms. In recent years the interest in how individuals evolve in the context of ecosystems is growing. This eco-evolutionary research needs model systems that can represent natural communities in their interactions and complexities, while still being a tangible model system.

In this thesis I investigated the microbial communities that are responsible for the fermentation and used the constituting bacteria to learn about bacterial community dynamics over time. This was done by combining three disciplines: 1) Food Microbiology, on the conversion of compounds by bacteria during spontaneous fermentation; 2) Evolution, on the changes in the fermenting community over time under selection pressure; 3 ) Ecology, on the roles and niches bacteria take within the fermenting community. This thesis had two aims. The first aim was to provide the first step towards the use of microbial communities of spontaneously fermented milk, such as Mabisi and Lait caillé, as model systems for studies on eco-evolutionary dynamics. For this, I performed a series of experiments with an increasing level of control and a decreasing level of complexity. These experiments started with producing Mabisi in traditional ways in the field to allow a study of bacterial dynamics, which resulted in a low level of control of variables. Later, I brought the microbial communities to the laboratory and used controlled environments without changing the diversity of the 
natural communities. The second aim was to provide ecological and evolutionary insights in Mabisi fermentations in the context of research for development. Here, the focus was on the practical application of bacterial dynamics for dairy fermentation. The potential use of the outcome for producers and consumers of spontaneously fermented foods by taking into account when constructing and interpreting the studies.

This thesis starts with a perspective on the use of microbial communities of spontaneously fermented products as model system for eco-evolutionary dynamics (Chapter 2). We outline what model systems are used for experimental testing of evolutionary theory so far, ranging from simple microbial communities in the laboratory and, more recently, to complex (natural) communities. Microbial communities from spontaneously fermented products bear several intrinsic advantages for executing evolution experiments: short generation times, small size and ability to be stored frozen and defrosted to perform competition experiments (fitness tests) between evolved and ancestral lines. Moreover, these natural microbial communities have a limited number of players and form an island of microorganisms that does not have a lot of influx from outside the confined system boundaries. There are several research questions with an evolutionary background that can be addressed using these microbial communities from fermented foods. This includes questions on changes species frequency in space and time, the diversity-stability relationship, niche space, fluctuating environment and community coalescence. Hypotheses on the influence of these factors on community evolution are described as well as a short descriptions of the experimental approach of such studies when microbial communities of spontaneously fermented foods are used.

Natural communities of Mabisi were analysed in a field experiment. A method was developed using filter paper disks for the preservation of DNA from diverse microbial communities for later analyses (Chapter $\mathbf{3}$ ). The bacterial species composition obtained through DNA extraction via the filter paper method showed sufficient resemblance to the composition obtained via traditional DNA extraction from the liquid milk sample. This method could therefore successfully be used to analyse diverse microbial communities from Mabisi in our remote field sites in rural Zambia. Field experiments were conducted to determine the effect of variations in fermentation vessels and types of back-slopping procedures on acidification and bacterial community composition during fermentation (Chapter 4). Due to high costs and a reduced availability of the traditionally used calabashes, nowadays more and more plastic containers are used for Mabisi production. However, the effect of this change in production practice on the properties of the product is unknown. Together with the local community, I have performed 15 fermentations, using two types of fermentation vessels (calabashes and plastic buckets) and three levels of back-slopping (active back-slopping, passive back-slopping and no back-slopping). In passive back-slopping, the bacteria that start the fermentation are transferred from the previous fermentation round via the fermentation vessel. During active back-slopping, finished product is transferred to raw material to inoculate the fermentation. Overall, bacterial communities decreased in diversity over time, where the drop in $\mathrm{pH}$ correlates with a decreased diversity, although the rate of acidification showed variation. In case of active back-slopping, the $\mathrm{pH}$ drop started right after inoculation. In the 'no backslopping' and 'passive back-slopping' fermentations, there was a clear lag phase before 
acidification started. No difference was found in bacterial diversity during and at the end of fermentation performed in plastic buckets or previously used calabashes. Besides small differences, all processing methods resulted in a microbial community dominated by Lactococcus lactis.

After the natural communities of traditional spontaneous fermentation were analysed, the next step was to bring the natural communities into the laboratory. Here, experiments were performed with lower complexity and an increased control. The species composition and metabolic profile of six different Mabisi samples were analysed before and after repeated propagation cycles (Chapter 5). These communities had similarity in their bacterial species composition and therefore had the potential to converge towards the same final species composition upon repeated propagation in a common environment. Species composition in all replicates propagated from the same original samples changed in a parallel way, yet that groups of communities derived from different original samples did not change in the same way. We observed that communities at the end of the repeated propagation cycles were either dominated by Lactobacillus helveticus or Lactobacillus delbrueckii. By modelling species compositions we tested the influence of four main factors on the species composition: initial species composition, selection imposed by the environment, selection caused by interaction between species and random processes in species dynamics. We found that the final species composition is mostly dependent on initial species composition, followed by random processes. The environment showed to have the least influence on the change in species composition.

We had the opportunity to work with a second spontaneously fermented dairy product, called Lait caillé. This traditional product originates from Senegal and is produced in wooden bowls, called lahals (Chapter 6). In terms of complexity and control, this experiment would position in between the field and laboratory experiments with Mabisi. The mode of propagation was traditional, meaning that the bacteria were transferred via the inside of the lahals that were used for repeated fermentations (passive backslopping). This traditional production method allowed us to study the natural bacterial communities in the lahals and translate our findings towards production practices in Senegal. We analysed the species composition of Lait caillé over time and added a probiotic starter containing Lactobacillus rhamnosus yoba 2012 to the traditional process attempting to enrich the bacterial species community in the final product. We found detectable levels of $L$. rhamnosus in the final products, which were dominated by Lactobacillus helveticus and Acetobacter species. The abundance of $L$. rhamnosus ranged between 0.2 and 1 percent of the total bacterial population, which is comparable to the concentration found in probiotic yoghurt. Subsequent rounds of fermentation using passive back-slopping without the addition of new L. rhamnosus led to a loss of this strain from the community of fermenting bacteria. Just as Mabisi it is an important part of the daily diet of men, women and children in rural and urban areas in Senegal. The addition of a health-promoting, probiotic bacterium to these products ensures the delivery of that probiotic activity to consumers. The addition of probiotic strains at every fermentation cycle can enrich the existing complex communities of traditionally fermented Lait caillé while traditional bacterial strains remain dominant in the bacterial communities. 
In conclusion, the bacterial communities found in the spontaneously fermented products show high diversity independent of production method. Our results show that even in a simple propagation environment and when confronted with new bacteria entering, these communities are able to stay diverse. The next step towards understanding these natural communities would be to construct simple communities based on combinations of individual species, while aiming to generate communities with similar characteristics, for example in terms of stability and aroma formation. The diversity we observed in the bacterial communities leads to functional stability as well as high levels product safety compared to fermented products with a lower species diversity. We found no differences in species diversity between Mabisi resulting from plastic buckets compared to calabashes, suggesting that other fermentation vessels are suitable to replace calabashes. Further, we found that the addition of a probiotic starter can enhance the nutritional value of the fermented product without losing the original fermenting community. These results will be used for improvements in production of traditionally fermented milk to ensure widespread availability of these products thereby improving public health. 


\section{Acknowledgements}

Luckily a PhD project is not something you have to do on your own. I have to thank many people that have supported me in the last 4,5 years, both on content basis as well as on an emotional level.

First of all a big thanks to Sijmen. You were always there for me with creative solutions and the ability to boost my confidence. Also a special thanks to Eddy for sharing your knowledge and motivating me. The support you both have given me was essential to get me to this point of having written my thesis. I am happy we could work together and I am looking forward to continue working with you! My other promotors, Bas and Anita, have been very important as well. Although maybe not so frequent, thank you both so much for giving me the right feedback on the right moment, to motivate me and to send me in the right direction! Also, besides for sharing their wisdom, I want to thank my team of promotors for building a friendly relationship in which I felt at home and safe to be myself and share my happiness and doubts.

My three Zambian men: Sydney, Justin and Bernard! It was a true delight to be able to work close together with you in the last 4,5 years. Thank you for explaining so much about Zambian culture and introducing me to the Zambian sense of humour! Joost, thank you for answering all my questions over and over again. Anna, it is amazing you joined the Mabisi research team; you brought some lady-power to the team!

During my project I was able to work together with some people from outside Wageningen University, which broadened my horizons and has been incredible useful in my work. A big thanks to the consortium members from the University of Zambia, Heifer Zambia, Yoba for Life and CSK as well as the collaborators in Path. My thesis would not have been the same without Sarah Majuru and the people from Mondake Dairy Association. I know it took a lot of nerve to let me into your household and family to teach me about your Mabisi production and maybe even more so to come to the Netherlands. We made memories I will never forget, thank you so very much for that!

To my brilliant students; Selen, Dechen, Juan and Janella, thank you for your insights and hard work. Working with bacteria sometimes asks for long days and returning to the lab in the weekend. This did not scare you away and the work you have done has inspired and helped me to continue the project. I hope these experiences will be just as helpful for you in your future careers. 
I cannot imagine having better colleagues than the people from genetics. Sabine, Ramon, Lennart, Margo and Kim, thank you for surrounding me both in time and space and with kindness and peer support. Working amongst you has made my days much more fun. Also I want to thank Bertha and Marijke, for helping me in the beginning of my PhD to find my way in the lab. Special thanks to Krithi, for joining me to the fries shop on evenings that I needed it. For all your kind words and strong character. I cannot find words for how much I miss you and how much I wanted us to live closer together. Thank you Arjan, Bart, Ben, Bwalya, Claudio, Corrie, Cris, Diego, Duur, Eric, Erik, Eveline, Florien, Fons, Francesco, Frank, Gabriella, Helena, Jelle, Jianhua, Jitpanu, Job, Joost K, Jordy, Jose, Klaas, Lennart, Lidia, Manja, Margreet, Mariska, Mark A, Mark Z, Mathijs, Philip, Phuong, Raphael, René, Robert, Roel, Sarah, Suzette, Tom, Valeria, Vanesa, Wouter and of course Wytske for giving me such an inspiring professional environment as well as joining in social activities, lab outing and WE-days! When moving on in my career, I will always think back of this amazing group!

Thank you, Margo, for everything you did for me in the last 4 years! I am so honoured you want to be my paranymph! Also Kristel, my other paranymph, your support has been amazing. I am so happy to call you my friend and you will always be the 'buuf' to my heart. Thank you for joining me on stage during my defence.

During my thesis I was lucky to meet many intelligent, interesting and fun people: through PPC, WPC, PhD trips in Italy and China, PE\&RC activities, courses and parties. Thank you all for sharing your insights and listening to my ideas. I am looking forward to meet you again during my future career or on the streets of Wageningen.

I want to thank my family for their interest in my project. Although I was sometimes unable to explain what I was doing and why, you never stopped inquiring about my progress. Knowing that there are people that support me no matter what, and that will be there for me unconditionally, made me brave enough to take up all the adventures and challenges life puts on my path.

In the last year before handing in my thesis, I could share all my doubts, frustrations and happiness with the most kind, funny and overall amazing guy. Thank you, Jelle, for standing next to me in moments where I do not know what to do, for being awkward with me (preferably in public), for taking away my fears, for sharing everything that is on your mind, accepting all my weirdness, for singing with me at every occasion and for dancing together like there is not tomorrow.

Birgit and Cho, thank you for being the BC to my A! There are no real Vrijmibo's without you. Thanks to all my other friends, from high school, study, Bont, Sweetbull, EMFS, and colleagues that became friends. I am privileged to be surrounded by people that I can join in adventures around the world, that we can cry together and celebrate together. So let's get this party started. 


\section{List of publications}

Adam E, Groenenboom AE, Kurm V, Rajewska M, Schmidt R, Tyc O, Weidner S, Berg G, de Boer W, Falcão Salles J. 2016. Controlling the Microbiome: Microhabitat Adjustments for Successful Biocontrol Strategies in Soil and Human Gut. Frontiers in Microbiology. 7.

Groenenboom AE, Smid EJ, Schoustra SE. 2019. Robust sampling and preservation of DNA for microbial community profiling in field experiments. BMC Research Notes. $12: 159$.

Groenenboom AE, Parker ME, Vries A de, Groot S de, Zobrist S, Mansen K, Milani P, Kort R, Smid EJ, Schoustra SE. 2019. Bacterial community dynamics in Lait caillé, a traditional product of spontaneous fermentation from Senegal. PLoS ONE. 14(5): e0215658. 


\section{About the author}

Anneloes was born on $31^{\text {st }}$ of October 1990 in Rotterdam, The Netherlands. She grew up in a small village called Poortugaal where she lived with her family for 18 years. After attending secondary education at Penta College CSG Angelus Merula in Spijkenisse she moved to Wageningen for a BSc Food Technology in 2009. During her BSc studies she followed a miner in Innovation and Entrepreneurship and did a thesis project at the Laboratory of Food Chemistry entitled "Purification of prenylated isoflavonoids from Rhizopus challenged soybean seedlings".

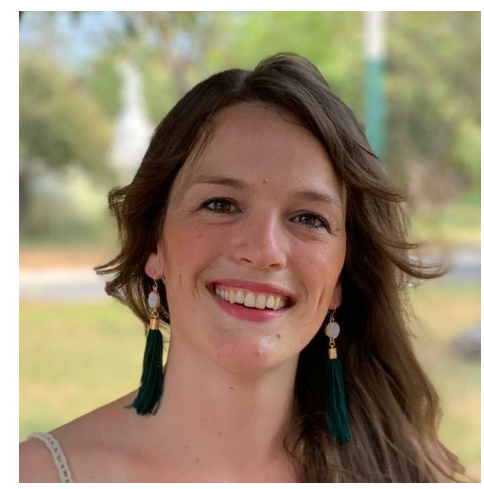

In 2012 Anneloes started the European Master in Food Studies, a Master programme in which she studied at leading universities in four European countries. She was part of a selected group of students who were privileged to receive training from top academic staff and respected experts in food industry. The first year she completed different courses and studies in Netherlands (Wageningen University \& Research), Ireland (university College Cork), France (AgroParisTech) and Sweden (Lund University). In the second year, she did a combined thesis internship project at Nestlé Product technology Centre in Konolfingen (Switzerland). The project was entitled "Increasing biomass production of Lactobacillus rhamnosus by implementing a pseudo fed-batch and a continuous system".

After completing her MSc studies, she got the opportunity to start her PhD project at the Laboratory of Genetics and Food Microbiology of Wageningen University \& Research in 2015. Her work is described in this thesis. During her PhD, Anneloes was an active member of the PE\&RC PhD Committee as well as the Wageningen PhD Committee. She helped in the organisation of various activities for her chair group, graduate school and university. 


\section{Overview of completed training activities}

PE\&RC Training and Education Statement

With the training and education activities listed below the PhD candidate has complied with the requirements set by the C.T. de Wit Graduate School for Production Ecology and Resource Conservation (PE\&RC) which comprises of a minimum total of 32 ECTS (= 22 weeks of activities)

\section{Review of literature ( 6 ECTS)}

Microbial communities from spontaneous fermented foods as model system to study eco-evolutionary dynamics

Writing of Project proposal (4.5 ECTS)

Ecology and evolution of microbial communities in Zambian fermented products

Post-graduate courses (3.1 ECTS)

Frontiers in microbial ecology; RSEE, PE\&RC \& SENSE (2015)

Food fermentation; VLAG (2016)

\section{Laboratory training and working visits (1.1 ECTS)}

PhD Trip North Italy: present at Parma university and visit University Bologna, University Milan, University Torino, EFSA, JRC Ispra etc. (2017)

Deficiency, refresh, brush-up courses (1.5 ECTS)

Basic statistics; PE\&RC (2015)

Competence strengthening / skills courses (4.5 ECTS)

Competence assessment; WGS (2015)

Mobilising your scientific network; WGS (2015)

Efficient writing strategies; WGS (2016)

Supervising MSc students; WGS (2017)

Career orientation; WGS (2018) 
PE\&RC Annual meetings, seminars and the PE\&RC weekend (3.7 ECTS)

PE\&RC First year, mid-term and last year weekend $(2015,2016,2018)$

WSG PhD workshop carousel (2015-2018)

PE\&RC day (2015-2018)

Wageningen career day (2017)

Discussion groups / local seminars / other scientific meetings (6.3 ECTS)

Experimental evolution discussion group (2015-2018)

The scientific spring meeting KNVM \& NVMM (2016)

Microbial population biology meetings (2016-2018)

Networks in ecology (NERN) (2016-2018)

International symposia, workshops and conferences (10 ECTS)

EMPSEB (2015)

ESEB (2017)

LAB12 (2017)

Evolution (2018)

Supervision of three MSc students (9 ECTS)

'Resilience of microbial communities in fermented dairy product Mabisi against invasion'

'Experimental evolution of a mabisi-inspired bacterial community'

'Change in the aromatic compounds formation in Mabisi due to a bacterium single omission in a synthetic community'

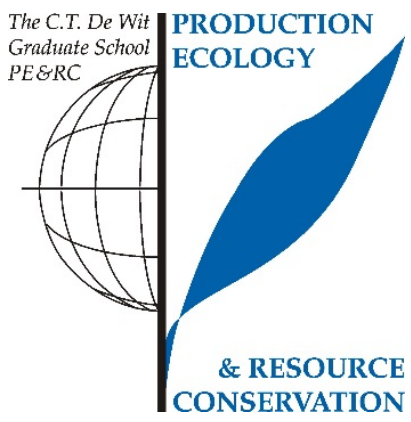




\section{Colophon}

The research of this thesis was carried out at the Laboratory of Genetics at the Wageningen University \& Research, The Netherlands as part of a project on Zambian traditional fermented products, which was financially supported by NWO-WOTRO in the Food \& Business Global Challenges Programme (W08.250.2013.108).

This project is a collaboration within Wageningen University \& Research with colleagues from Food Quality and Design and Food Microbiology as well as with other partners in the Netherlands (CSK Food Enrichment and Yoba-for-Life) and in Zambia (University of Zambia, Tropical Diseases Research Centre, Heifer International).

Cover design by: Kelley Leung

Thesis layout by: Anneloes Groenenboom

Printed by: GVO drukkers \& vormgevers 